\title{
Aerodynamic Analysis of Multistage Turbomachinery Flows in Support of Aerodynamic Design
}

\author{
John J. Adamczyk \\ NASA Lewis Research Center \\ Cleveland $\mathrm{OH} 44135$
}

\begin{abstract}
This paper summarizes the state of 3D CFD based models of the time average flow field within axial flow multistage turbomachines. Emphasis is placed on models which are compatible with the industrial design environment and those models which offer the potential of providing credible results at both design and off-design operating conditions. The need to develop models which are free of aerodynamic input from semi-empirical design systems is stressed. The accuracy of such models is shown to be dependent upon their ability to account for the unsteady flow environment in multistage turbomachinery. The relevant flow physics associated with some of the unsteady flow processes present in axial flow multistage machinery are presented along with procedures which can be used to account for them in 3D CFD simulations. Sample results are presented for both axial flow compressors and axial flow turbines which help to illustrate the enhanced predictive capabilities afforded by including these procedures in 3D CFD simulations. Finally, suggestions are given for future work on the development of time average flow models.
\end{abstract}

\section{Introduction}

Turbomachinery aerodynamicists have long realized that the flow within multistage turbomachinery is complex. In addition to the small-scale chaotic unsteadiness due to turbulence, which is nondeterministic, at a large scale the flow is also unsteady and aperiodic from blade passage to blade passage. The flow features associated with these large scales are deterministic. It is because of the unsteady deterministic flow that turbomachinery is able to either impart or extract energy from a flow. The term unsteady deterministic is used to characterized all unsteady behavior linked to shaft rotational speed. The length and time scales associated with the unsteady flow field within turbomachinery are understood. The range of these length and time scales is large and consequently turbomachinery flow modeling is very challenging. The challenge is to develop an analytical model of sufficient fidelity to address key design issues. At the same time, the cost and time of executing a simulation based on the model, and the cost of acquiring and maintaining the cmpirical database which underpins the model must be compatible with the design environment. The balance point between model resolution and the cost of implementation and execution is constantly changing as the cost of computing comes down and more detailed experimental information is gathered about the nature of the flows within multistage turbomachinery. This change in the balance point with time must also be considered when deciding upon a mathematical modeling strategy.

Figure (1) illustrates a range of mathematical models which can be used to simulate turbomachinery flows. They appear on the figure in the order of their fidelity. At the extreme left are the Navier-Stokes equations, at the extreme right are the equations governing a mean-line model. The other models which appear on the figure will be addressed in the text.

The balance between model resolution and cost of model implementation can be illustrated with the aid of Fig. (1). Apart from the empirical information required to model the physical and thermodynamic properties of a fluid, no other empirical information is required to solve the Navier-Stokes equations. The direct solution of the Navier-Stokes equations for Reynolds numbers typical of aero and industrial turbomachinery configurations would produce a nondeterministic or turbulent flow state. Although the fidelity of such a simulation would be very impressive, the task of extracting information to guide an aerodynamic design would be difficult. In addition, the computer requirements associated with solving the Navier-Stokes equations in support of aerodynamic design, both in terms of CPU speed and memory size, would far exceed the capabilities of today's most advanced computers. Otherwise, the cost of acquiring the empirical database for the physical and thermodynamic . properties which underpin the Navier-Stokes equations for working fluids typical of most turbomachinery applications 
is reliatively modest and, for the most part, is readily available: todiay.

At the other extreme, the computer requirements associated with exccuting a simulation based on a mean-line model of a multistage configuration is well within the capabilities of personal computers. The fidelity of a mean-line model is one dimensional in space and may include time as an additional independent variable. The empirical data base underpinning a mean-line model used in turbomachinery aerodynamic design is large. The maintenance and acquisition cost associated with this database is also large.

In the foreseeable future, it is highly unlikely that computers of sufficient power for use in solving the NavierStokes equations for a multistage turbomachinery configuration will be readily available. Although mean-line models are very useful in preliminary aerodynamic design, they are insufficient for detailed aerodynamic blade design of advanced configurations. For these reasons, aerodynamicists have evolved models whose resolution lies between these two extremes. A number of these models are noted on Fig. (1).

As of ten years ago the aerodynamic design of most axial flow multistage turbomachinery was executed using various axisymmetric flow models. The use of these models was iterative. A design was executed based on the existing database. Data obtained from tests of the fabricated hardware was used to update empirical correlations embedded within these models. The updated axisymmetric flow model was then used to support the next design of the configuration. This boot-strap approach to the aerodynamic design of axial flow multistage turbomachinery resulted in some truly impressive machines as evidenced by the aerodynamic performance they achieved. However, because of strong economic forces the turbomachinery industry was forced to re-examine this iterative approach to aerodynamic design. These economic forces mandated that industry reduce the time and cost of developing a turbomachinery component and, at the same time, required the design of machinery whose performance goals lay outside the then existing experience base. As a result, a strong need arose for developing a new methodology for executing the aerodynamic design of axial flow multistage turbomachinery. The foundation of this new methodology required aerodynamic models whose resolution was greater than that of the axisymmetric flow models. These models had to allow for overnight turnaround using computer resources compatible with the design environment. Finally the models had to be capable of addressing the aerodynamic issues associated with the design of advanced configurations.

The objective of this paper is to review the development and application of 3D computational fluid dynamics (CFD) based models for use in the aerodynamic design of multistage axial flow turbomachinery. More specifically, this review discusses those models which offer the potential of providing credible results at both design and off-design operating conditions without recourse to aerodynamic matching information supplied implicitly or explicitly by existing aerodynamic design systems. The term aerodynamic matching refers to the matching of the inlet flow requirements of a stage to the outlet flow of upstream stages.

The paper is divided into four parts. The first part discusses the historical development of the models currently used in the design of multistage turbomachines. The second part of the paper deals with the issues associated with modeling the time-average flow within a typical passage of a blade row embedded in a multistage turbomachine. The third section presents results from numerical simulations of multistage axial flow compressors and turbines based on a three-dimensional flow model which attempts to account for the unsteady flow environment within these machines. The last section summarizes the results presented in the paper and suggests areas for future research. An appendix is included in which a mathematical procedure is outlined for constructing the field equations governing the time average flow within a typical passage of a blade row embedded in an axial flow multistage turbomachine.

\section{Historical Background}

Historically in order to make any headway in analyzing multistage turbomachinery flows it was necessary to assume that each blade row had an infinite number of blades. If the incoming and exiting flow is axisymmetric and independent of time, the assumption relating to blade count implies that the entire flow-field is axisymmetric. The resulting flow-field is in general three-dimensional, since the radial, tangential, and axial velocity components all exist. This axisymmetric flow representation is described by throughflow models. The individual blade rows are represented by a force distribution (actuator duct model) and an energy source (for compressors) or energy sink (for turbines) distribution. If the chord length of each blade row is allowed to vanish while the aerodynamic loading on the blade row is maintained, the blade row representation is that of an actuator disk. Across each disk, the tangential velocity component undergoes a finite change due to the tangential force generated by the blade row.

The axisymmetric flow-field is treated as a core region and two endwall regions. The effect of the endwall regions on the core region is, in part, accounted for by means of a flow blockage. It is assumed that the blade force acting on the core fluid can be obtained from cascade flow theory, Marble (1964), while the force acting on the fluid within the endwalls is based on empirical correlations, Smith (1969). . As of ten years ago the through-flow models were the mainstay of aero design systems for multistage axial flow turbo- 
marthines. Todlay their primary use is in preliminary design to gencrate blade shapes. An excellent review of the devrilopurnt of through-flow and cascade flow theory is given by Servoy (1981). In addition, a thorough discussion of the underlying flow physics being accounted for in through-flow models is presented by Cumpsty (1989).

It was not until digital computers were easily accessible to turbomachinery flow modelers and the development of CFD that the through-flow models were replaced by quasi-three dimensional flow models. The quasi-threedimensional flow models numerically coupled an axisymmetric flow model to a cascade flow model. The formulation of these quasi-three-dimensional models was influenced by the work of Wu (1952). The axisymmetric flow model provided the stream surfaces on which the cascades were defined. The cascade flow model provided the blade force distribution required as input to the axisymmetric flow model. The development of such a flow model is outlined by Jennions and Stow (1986). Two more recent publications, Howard and Gallimore (1993) and Gallimore (1998), are representative of the current state of the art of axisymmetric flow models.

The quasi-three-dimensional flow models were an advancement over the through-flow models. However, like the through-flow models, the quasi-three-dimensional models still required a sound estimate of the aerodynamic blockage for the predictions to match data. This limited a new design to regions of design parameter space which was still relatively close to that of a previous machine. Without a good a priori estimate of the blockage associated with a design, aero designs executed using the quasi-threedimensional flow model fell short of their performance goals. This led to rebuilds of machinery, a costly and time consuming process.

In Fig. (1), these quasi-three-dimensional flow models are grouped together with the through-flow models under the label axisymmetric flow models. Because the axisymmetric flow models are of higher fidelity, they lie to the left of the mean-line models.

Within the last ten years there has been a steady infusion of 3D CFD-based models into axial flow multistage turbomachinery design systems. In part this has been due to the useful role these models have played in the analysis of isolated blade rows. The performance level of the fan rotor of the current generation high bypass ratio turbofan engine is directly tied to the advances made in the use of 3D CFDbased models to guide aero design. The initial fan rotor geometry may be provided by a through-flow or a quasithree-dimensional system but the final shape of the rotor is evolved exclusively using 3D CFD-based models. The rotor shape is tailored to control the interaction of shock waves with the blade surface boundary layers to avoid separation and to minimize loss. In addition using these 3D CFD models one is able to resolve the tip clearance flow as well as the stator hub leakage flow, and establish their impact on aerodynamic performance. From this activity guidelines have been established for use in aerodynamic design, Khalid, et al. (1998), Van Zante, et al. (1999), Wellborn, et al. (1999).

The 3D CFD-based models which were initially introduced into multistage axial flow turbomachinery design systems ignored the impact of the unsteady, deterministic flow which exists within axial flow multistage turbomachines. The unsteady, deterministic flow is produced by the surrounding blade rows and its frequency content is linked to shaft rotational speed. These $3 \mathrm{D}$ models have two forms. The first is simply an isolated 3D blade row CFD-based model linked to a through-flow code. The initial geometry and the inlet and exit flow conditions to the blade row being designed are established by the through-flow or quasi 3D system. The established inlet and exit flow conditions to a blade row set the boundary conditions for the 3D model. 3D simulations are executed with geometry updates until a blade configuration is found whose inlet and exit flow closely matches that of the through-flow system. The aerodynamic matching of stages within a machine is established by the through-flow system.

The second model pioneered by the work of Denton and Singh (1979), employs 'Mixing Planes' between blade rows to define a steady inlet and exit flow for each blade row within a machine. The mixing plane model produces a series of blade passage flow-fields, each of which is independent of time and periodic in the tangential direction, with a period equal to the pitch of the respective blade row. This model is strictly valid in the limit as the axial spacing between blade rows becomes large. Further details on the mixing plane model are provided in the work of Dawes (1992), and Hall (1993).

In using the mixing plane model in aerodynamic design the initial geometry is provided by a through-flow system. Generally information is also provided implicitly or explicitly detailing the aerodynamic matching of stages within a machine. This information is provided by the through-flow system, Gallimore (1998), (1999). Simulations of groups of stages or the entire machine are executed with geometry updates to remove any undesirable flow features associated with the initial configuration. However, during this process the aerodynamic matching of stages within the machine remains essentially that established by the through-flow system.

Key to the success of designs evolved using either approach is the aerodynamic matching of stages as established. by the through-flow system. Since the aerodynamic matching of stages is a function of flow blockage, these two ap- 
proakches implicitly rely on the through-flow system, and not the 3D CFD based models, to establish the flow blockage within a machine.

There are two approaches which account for the unsteady deterministic flow environment within a multistage turbomachine. The first of these approaches directly simulates the unsteady deterministic flow state. The equations associated with this model are the Reynolds average form of the Navier-Stokes equations. The use of this model in design applications where overnight simulation turnaround is mandated must await the development of more powerful computers. However, recent publications, Chen, et al. (1994), Dorney, et al. (1990), Giles, (1990), Gundy-Burlet, (1993), Hodson and Dawes (1998), (1993), Rai and Dring, (1987), and Sharma, et al. (1992), have shown these models to be very useful in conducting numerical experiments to quantify the impact of the unsteady deterministic flow on turbomachinery aerodynamic performance and durability. They have also proven useful in generating data bases for use in the development of models which introduce the effect of the unsteady deterministic flow environment into simulations of the time average flow field within multistage turbomachines, Hall (1997).

The remaining approach is based on the flow model derived from the work of Adanczyk (1985). This model and its off-shoots have proven useful in design applications, LeJambre, et al. (1998). The model developed by Adamczyk (1985) is referred to as the average-passage flow model. This flow model describes the time average flow field within a typical passage of a blade row embedded within a multistage configuration. The resulting flow field is periodic over the pitch of the blade row of interest. The averagepassage flow model is an analysis model as are all the others which have been referred to thus far. This means that geometry is the input and the output is the flow field generated by the geometry. When using the average-passage flow model in aerodynamic design, the initial geometry is defined by a through-flow system. During the design process geometry updates are done exclusively based on simulation results from the average-passage model, Mansour (1999), Wellborn (1999). No output from an axisymmetric through-flow system which implicitly or explicitly sets the aerodynamic matching of stages within a turbomachine is provided to the average-passage simulations. The credibility of an average-passage flow simulation is not tied to aerodynamic matching information provided by a through-flow system or to input estimates of flow blockage. The credibility is tied to the models used to account for the effects of the unsteady flow environment on the average- passage flow field. The effect of the unsteady deterministic flow field on aerodynamic matching of stages is accounted for by velocity correlations within the momentum equations associated with the average-passage flow field and by a velocity total enthalpy correlation within the energy equation associated with the average-passage flow field. The term unsteady deterministic refers to all time dependent behavior linked to shaft rotational speed. All unsteady behavior not linked to shaft rotational speed is referred to as nondeterministic. The mathematical steps leading to these correlations are outlined in Appendix A.

There is no doubt that 3D CFD based models with aerodynamic input from through-flow models have provided credible designs. These models will continue to provide credible designs as long as the design parameters are within the bounds of the database underpinning well-calibrated through-flow or quasi 3D design systems. However, reliance on through-flow or quasi 3D models to set key aerodynamic design parameters such as the aerodynamic matching of stages greatly impedes the utility of these models. Specifically, they are limited in their ability to uncover the fluid mechanics which is controlling the performance of multistage axial flow turbomachines at design and off-design operating conditions. The objective of 3D CFD based modeling should be to break free of the dependence on throughflow or quasi 3D design systems except for providing the initial geometry. If such 3D CFD based models are proven credible, their use will allow aerodynamic designs of machinery whose parameters lay outside the bounds of the database underpinning through-flow systems to be executed with confidence. This would include both design and offdesign operation, and in the case of compressors, adequate stall margin. This author believes that such 3D CFD based models can be developed within the framework of a time average flow model by correctly accounting for the effects of the unsteady flow environment within a multistage axial flow turbomachine.

This section is concluded by providing a simple example which illustrates the impact of an unsteady, deterministic flow on the time average flow field. Consider the flow field downstream of an isolated compressor rotor. In the reference frame of the rotor the flow is steady in time. In the absolute or stator frame of reference the flow is unsteady in time, the result of the rotor flow field being nonaxisymmetric. Figure (2) shows the time average and mass average total temperature distributions in the stator frame of reference at two axial locations downstream of NASA Rotor 37, Reid and Moore (1978), as a function of span. These results were derived from a simulation at design speed and near peak efficiency. The axial locations Station 3 and Station 4 correspond to stations reported in the ASME blind test case exercise, Denton (1996). These two locations are approximately one rotor chord apart. Station 3 would be . slightly upstream of a stator had a stator been included in the simulation. The difference between the time average 
and mass averaged total temperature distribution downstream of the rotor is a direct measure of the unsteady total temperature exiting the rotor. At Station 4 the difference is small indicating that the unsteady flow field is effectively mixod out at this location while at Station 3 the difference is significant. Figure (3) shows the footprint of fluid particles on an axial plane at Station 4 which were released at Station 3 at $20 \%$ of span, $50 \%$ of span and $80 \%$ of span. The particles which are in the rotor wake and in the core fluid are noted in the figure. The particles in the wake have a radial drift velocity relative to the particles in the core fluid. The wake particles also have an excess of total temperature relative to the core fluid particles. The radial drift of the wake particles relative to the core fluid particles is an unsteady deterministic process when viewed in the absolute or stator frame of reference. This process results in a redistribution of the time average total temperature field between Stations 3 and 4 . The difference between the time average total temperature distribution at Station 3 averaged over the span and the distribution at Station 4 averaged over span is nearly $7 \%$ of the total temperature rise across the rotor. This difference is not insignificant.

Figure (2) also shows that there is a difference in the spanwise distribution of the mass average total temperature at Stations 3 and 4 even though the mass average total temperature across the flow annulus is conserved. Note the difference in the two mass average total temperature profiles outboard of $80 \%$ span. The change in either the time average total temperature profiles or the mass average total temperature profiles between Stations 3 and 4 is not the result of transport by the time average velocity field. Transport of either the time average or mass average total temperature at Station 3 by the time average velocity field would yield a profile only slightly altered from that at Station 3. The change in the total temperature profiles which occurs between Stations 3 and 4 is the direct result of the unsteady deterministic flow exiting the rotor. Had a downstream stator been located in the flow field at an axial location downstream of the rotor typical of core engine compressors the spanwise redistribution of the time average total temperature field would be taking place within the stator passages. The time average total temperature field exiting the stator would be influenced by the spanwise migration of the rotor wake fluid particles and thus impact the performance of a downstream rotor.

The cumulative effect of spanwise redistribution of total temperature has a significant impact on the predicted aerodynamic performance of axial flow multistage compressors and turbines.

The next section presents a number of models by which one can account for the effects of the unsteady deterministic flow state on the time average flow field and the flow physics being captured by these models.

\section{Flow Physics Associated With the Average- Passage Stress and Total Enthalpy Flux}

In Appendix $\mathrm{A}$ it is shown that within the framework of the average-passage model the average-passage stress and total enthalpy flux account in part for the effect of momentum transport and total enthalpy transport attributed to surrounding blade rows. The average-passage stress appears in the average-passage form of the momentum equations, while the average-passage total enthalpy flux appears in the average-passage form of the energy equation. From Appendix A the average-passage stress and total enthalpy flux are:

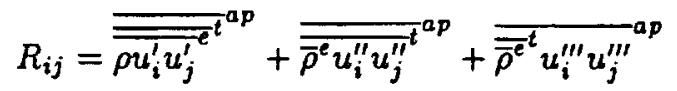

and

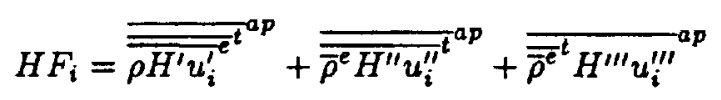

where lower case $u_{i}^{\prime}$ is the velocity component in $i_{t h}$ direction, $H$ is the total enthalpy, and $\rho$ is the fluid density. The remaining variables which appear in Eqns. (A9) and (A10) are defined in Appendix A.

The average-passage stress and the average-passage total enthalpy flux, are composed of three terms. With respect to a given blade row, the first term is due to the non-deterministic flow field, the second is due to the unsteady deterministic flow field, and the third is due to the average-passage flow field of blade rows other than the one of interest which are in the same spatial reference frame. Thus the first term in Eqns. (A9) and (A10) accounts for the diffusion of total enthalpy and momentum attributed to unsteady flow processes not linked to shaft rotational speed, which includes diffusion due to turbulence. Since in most turbomachines the flow is turbulent, the need to model this portion of the average-passage stress and the corresponding portion of the average-passage total enthalpy flux is selfevident.

The component of the average-passage total enthalpy flux associated with the unsteady deterministic flow field is the result of flow processes directly linked to shaft rotational speed. These flow processes are responsible for the radial redistribution of total temperature previously illustrated. They are also responsible for the circumferential redistribution of total temperature. This redistribution of total temperature leads to the formation of hot spots in turbines which has a significant impact on turbine blade life.

The component of the average-passage stress associated with the unsteady deterministic flow is responsible for the , recovery of wake mixing loss in compressors, flow blockage, and the spanwise redistribution of momentum. 
The remaining portion of the average-passage stress and total enthalpy flux accounts for the effect of blade indexing (i.e. the relative circumferential placement of blade rows which are on the same shaft). It is through these terms that the effect of circumferential placement of hot streaks entering a turbine blade row and the impact of indexing on blade loss are accounted for.

The impact of the unsteady deterministic flow on the aerodynamic performance of an axial flow multistage turbomachine is the main topic of this section. The terms which account for this are the second expressions in Eqns. (A9) and (A10):

$$
R_{i j}^{D}={\overline{\overline{\bar{\rho}}^{e} u_{i}^{\prime \prime} u_{j}^{\prime \prime}}}^{a p}
$$

and

$$
H F_{i}^{D}={\overline{\overline{\bar{\rho}}^{e} H^{\prime \prime} u_{i}^{\prime \prime}}}^{a p}
$$

The superscript $D$ ascribed to $R_{i j}^{D}$ and $H F_{i}^{D}$ denotes the deterministic component of the average-passage stress and the deterministic component of the average-passage total enthalpy flux. All of the terms which appear on the righthand side of Eqns. (1) and (2) are associated with the unsteady deterministic flow.

The unsteady flow field is defined with respect to the stator frame of reference, unless otherwise noted. The ensuing discussion is equally applicable to the unsteady flow field with respect to the rotor frame and therefore no separate discussion is warranted. With respect to the stator frame of reference the unsteady deterministic velocity and total enthalpy in Eqs. (1) and (2) may be decomposed as follows:

$$
\begin{aligned}
u_{i}^{\prime \prime} & =\tilde{u}_{i}(z, r, \theta-\Omega t)+\widehat{u}_{i}(z, r, \theta-\Omega t) \\
& +\widetilde{\tilde{u}}_{i}(z, r, \theta, t) \\
\tilde{u}_{i} & =\sum_{n=1}^{N}\left[U_{i_{(n)}^{a p}}^{a p}-u_{i}^{a x i}(z, r)\right]
\end{aligned}
$$

and

$$
\begin{aligned}
H^{\prime \prime} & =\widetilde{H}(z, r, \theta-\Omega t)+\widehat{H}(z, r, \theta-\Omega t) \\
& +\widetilde{\widetilde{H}}(z, r, \theta, t)
\end{aligned}
$$

where

$$
\tilde{H}=\sum_{n=1}^{N}\left[H_{(n)}^{a p}-H^{a x i}(z, r)\right]
$$

The subscript $n$ denotes the $n_{t h}$ rotor blade row of which there are N. The first term in Eqn. (3) and (4) is a sum of the non-axisymmetric component of the average-passage vclocity and total enthalpy field attributed to each rotor. The second term in Eqn. (3) and (4), superscript, denotes the unsteady flow field arising from the circumferential indexing of rotor blade rows. For a single stage machine this term is absent. The remaining term arises because of unsteady rotor-stator flow interactions. The first two terms in Eqn. (3) and (4) are independent of time in the rotor frame of reference, while the last term is a function of time in both the stator and rotor frame of reference.

The correlation, Eqn. (1), is constructed by forming the product of the unsteady deterministic velocity with itself and density. The constructed product is first averaged over time according to Eqn. (A2) and then averaged according to Eqn. (A4) which yields its average-passage form. The same procedure is used to construct the correlation in Eqn. (2). The development of a scheme to evaluate all of the terms in correlations Eqn. (1) and (2) is a formidable task which is far beyond the objective of this paper. The evaluation of all terms is also unnecessary at the present time. What is necessary, and the focus of this section, is to identify which terms are deemed to be the most important and address the modeling of these terms. The next section shows what new predictive capability results from including the models developed in this section in the simulation of axial flow multistage turbomachines.

The prioritization of the terms in correlations Eqn. (1) and (2) formed from Eqns. (3) and (4) is based on the premise that the further blade rows are apart the less their flow interactions contribute to the correlations. This premise appears reasonable as evidenced by the plots in Fig. (2). At station 4, which is slightly downstream of the trailing edge of a stator had it been placed in the flow path, the unsteady flow is nearly mixed-out as evident by the correspondence between the spanwise distribution of the mass average total temperature and the time average total temperature. Based on the stated premise, the terms deemed most important are the terms which are the self product of the non-axisymmetric component of the average-passage velocity field of an individual blade row and the corresponding total enthalpy velocity product. The next series of terms involve products which contain the rotor-stator interaction components, $\widetilde{\widetilde{u}}_{i}(z, r, \theta, t)$ and $\widetilde{\widetilde{H}}(z, r, \theta, t)$. These remaining terms at the present time are thought to be of less importance. The resulting unsteady flow field upon which the correlations are estimated is that produced by a single rotor interacting with an upstream stator and a downstream stator, the unsteady flow interactions with the upstream stator are independent of the unsteady flow interactions with the downstream stator. With respect to the unsteady flow field in the rotor frame of reference the unsteady flow field upon which the correlations would be based is that produced by 
a stat,or with a rotor on either side. Similarly, the unsteady interiution with the upstream rotor is assumed to be independent of the unsteady interaction with the downstream rotor.

In the stator frame of reference the resulting contribution from the $n_{t h}$ rotor to the correlations Eqn. (1) and (2) simplifies to:

$$
\begin{aligned}
& R_{i j}^{(n)}={\overline{\overline{\bar{\rho}^{e} \widetilde{u}_{i_{(n)}} \widetilde{u}_{j_{(n)}}}}}^{a p} \\
& +{\overline{\overline{\bar{\rho}^{e}\left(\tilde{u}_{i_{(n)}} \tilde{\widetilde{u}}_{j}+\tilde{\tilde{u}}_{i} \tilde{u}_{j_{(n)}}\right)}}}^{a p}+{\overline{\overline{\bar{\rho}^{e} \widetilde{\widetilde{u}}_{i} \widetilde{\widetilde{u}}_{j}^{t}}}}^{a p}
\end{aligned}
$$

and

$$
\begin{aligned}
& H F_{i}^{(n)}={\overline{\overline{\bar{\rho}^{e} \tilde{H}_{(n)} \bar{u}_{i_{(n)}}}}}^{a p}
\end{aligned}
$$

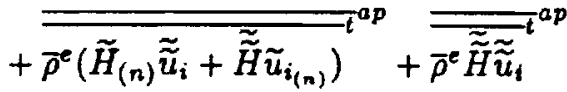

with $\tilde{u}_{i_{(n)}}$ defined as,

$$
\widetilde{u}_{i(n)}=U_{i_{(n)}}^{a p}-u_{i}^{a x i}
$$

The time average of the first term in Eqn. (5) is axisymmetric as is the time average of the corresponding total enthalpy velocity product. A procedure for estimating either of these terms was presented by Adamczyk, et al. (1986), and a generalization of the procedure is given by Kirtley and Turner (1999). A variation of the procedure derived by Adamczyk, et al. (1986), is presented by Rhie, et al. (1998). All three of these research groups have shown the benefits of incorporating their respective procedures for estimating the first term in Eqns. (5) and (6) into 3D CFD codes used in the simulation of axial flow multistage turbomachinery. It is through these terms that the effect of spanwise redistribution of momentum and total enthalpy due to the unsteady deterministic flow field is accounted for in modeling the average-passage flow field. These terms also insure that the time average vorticity field and time average momentum flux entering a stator are consistent with the time average velocity field, Adamczyk (1991), and by doing so account for the flow blockage of incoming rotor wakes. The second and third correlations in Eqns. (5) and (6) account for rotor-stator unsteady flow interactions such as the transport of a rotor wake across a compressor stator passage and its subsequent pile-up on the stator pressure surface. In the case of a turbine vane these correlations account for the pile-up of rotor wakes on the vane suction surface.

\section{Spanwise redistribution of total enthalpy and momentum}

In the next three sections the fluid mechanic processes which are described by the average-passage stress and total enthalpy flux are explored in more detail. The first term in Eqn. (6) formed from the product of the non axisymmetric portion of the average-passage velocity field and the non axisymmetric portion of the average-passage total enthalpy field accounts for the spanwise redistribution of the time average total temperature field shown in Fig. (2). To show this, consider the flow field downstream of the rotor which includes a stator. Assume that the flow is deterministic, adiabatic and inviscid, and that the fluid is a non-conducting ideal gas. The energy equation for this unsteady flow is:

$$
\frac{D H}{D t}=\frac{1}{\rho} \frac{\partial p}{\partial t}
$$

where $H$ is the absolute total enthalpy of the unsteady deterministic flow field, and $\vec{u}, p$, and, $\rho$ are the velocity, pressure and density of the unsteady deterministic flow field. $\frac{D}{D t}$ is the material time derivative and $t$ is time. Combining Eqn. (8) with the continuity equation,

$$
\frac{\partial \rho}{\partial t}+\vec{\nabla} \cdot \rho \vec{u}=0
$$

yields,

$$
\left[\frac{\partial \rho H}{\partial t}+\vec{\nabla} \cdot \rho H \vec{u}\right]=\frac{\partial p}{\partial t}
$$

The time average of Eqn. (10) is

$$
\vec{\nabla} \cdot{\overline{\rho T_{0} \vec{u}}}^{t}=0
$$

where $H$ has been replaced by the product of the specific heat at constant pressure, assumed to be a constant, and total temperature $T_{0}$. Equation (11) states that the mass-average total temperature of the unsteady deterministic flow field is conserved across the stator.

$T_{0}$ and $\vec{u}$ can both be decomposed into a time average component and an unsteady component:

$$
\begin{aligned}
& T_{0}={\widetilde{T_{0}}}^{t}+T_{0}^{\prime \prime} \\
& \vec{u}=\overrightarrow{\vec{u}}^{t}+\vec{u}^{\prime \prime}
\end{aligned}
$$

The total temperature $\widetilde{T}_{0}^{t}$ and the velocity $\widetilde{\mathbb{u}}^{t}$ are density weighted, Adamczyk (1984). Introducing both decompositions into Eqn. (11) yields:

$$
\vec{\nabla} \cdot \vec{\rho}^{t}{\widetilde{T_{0}}}^{t} \widetilde{\vec{u}}^{t}=-\vec{\nabla} \cdot \overline{\rho T_{0}^{\prime \prime}}{\overrightarrow{u^{\prime \prime}}}^{t}
$$

For an ideal gas with constant thermodynamic properties, . the correlation on the right-hand side of Eqn. (14) multiplied by the specific heat at constant pressure is equal to 
Eqn. (2) (i.e. the portion of the average-passage total enthalpy flux associated with the unsteady deterministic flow tiold).

If the divergence of the time average total temperature Hux associated with the unsteady deterministic flow field is nonzero, Eqn. (14) implies a spatial variation in the time average total temperature will exist even if the incoming time average total temperature field is uniform. The time average total temperature distribution at Station 3 and Station 4 in Fig.(2) differ because of the existence of the time average total temperature flux associated with the unsteady deterministic flow field and its spatial variation. For the same reason the mass average total temperature profiles also differ.

To proceed further with this analysis assume the time average total temperature distribution entering the stator is that at Station 3 in Fig. (2). Figure (4) shows a snap shot at an instant in time of this total temperature field projected onto an axial plane. The figure shows that a fluid particle in the wake has a higher total temperature than a neighboring fluid particle in the core flow, this being the result of the wake fluid particle having taken longer to pass through the rotor. Figure (4) also shows a sketch of the time history of this total temperature distribution along with the time history of the radial velocity at Station 3. Recall that Fig. (3) showed that the fluid particles in the rotor wake drifted radially relative to neighboring fluid particles in the core flow. With respect to the time history of total temperature sketched in Fig. (4), the unsteady total temperature component, $T_{0}^{\prime \prime}$, may also be written as:

$$
T_{0}^{\prime \prime}=\widehat{T_{0}}-{\widetilde{T_{0}}}^{t}+T_{0_{d}}
$$

where $\widehat{T_{0}}$ is the total temperature of the freestream fluid (assumed to be independent of time) and $T_{0 d}$ is the excess of total temperature within the wake. Introducing Eqn. (15) into the right hand side of Eqn. (14) yields:

$$
\vec{\nabla} \cdot \bar{\rho}^{t}{\widetilde{T_{0}}}^{t} \overline{\vec{u}}^{t}=-\vec{\nabla} \cdot \overline{\rho T_{0 d}{\overrightarrow{u^{\prime \prime}}}^{t}}
$$

In deriving Eqn. (16) one must note that the time average of the product of the density and the velocity component $\vec{u}$ is zero. The correlation on the right-hand side of Eqn. (14) has been rewritten as a correlation involving the excess of total temperature within the wake and the unsteady velocity.

Equation (16) is valid upstream of the stator but is also valid within the stator passage provided a fluid particle maintains its total temperature or nearly does so as it convects through the stator. According to Eqn. (6), the unsteady velocity which appears in the correlation on the right-hand side of Eqn. (16) has two parts, one associated with the non-axisymmetric component of the averagepassage velocity field of the upstream rotor, $\tilde{u}_{i_{(n)}}$, and the other resulting from the interaction of the wake with the stator, $\tilde{\tilde{u}}_{i}$. Away from the surface of a stator blade $\widetilde{u}_{i_{(n)}}$ dominates. Near the surface both are of the same order of magnitude. For an inviscid fluid their component normal to the stator surface is of equal magnitude but of opposite sign. For a viscous fluid, at the stator surface these components are of equal magnitude and of opposite sign.

A way from the surface of a stator blade, but near an endwall, the derivative normal to the endwall of the correlation involving the component of $\tilde{u}_{i_{(n)}}$ normal to the endwall and $T_{0 d}$ is the dominant term on the right-hand side of Eqn. (16). If the endwalls are right cylindrical surfaces the resulting term is the radial derivative of the correlation involving the radial component of $\tilde{u}_{i_{(m)}}$ and $T_{0 d}$. With reference to Fig. (4) $T_{0 d}$ is positive. The time average of the product of the density, $T_{0 d}$, and the radial component of $\tilde{u}_{i_{(n)}}$ is also positive. At an endwall this correlation is zero, the result of the normal component of $\tilde{u}_{i_{(n)}}$ being zero at the endwall. Thus at the shroud the radial derivative of the correlation formed by the density, $T_{0 d}$ and the normal component of $\tilde{u}_{i_{(n)}}$ is negative. At the hub the radial derivative of this correlation is positive. A positive radial derivative implies an energy sink while a negative radial derivative implies an energy source. The sink and source formed by the radial derivative of the portion of the average-passage total enthalpy flux associated with the unsteady deterministic flow field result in a redistribution of the time average total temperature field in the stator frame of reference. In the present example it would lead to an increase in the time average total temperature at the shroud and to a decrease at the hub. This would accentuate the total temperature profile which is opposite to the trend generally associated with turbulent diffusion. With respect to Fig. (2) the radial derivative of the correlation between the radial component of $\tilde{u}_{i_{(n)}}$ and $T_{0 d}$ is negative outboard of $70 \%$ span and positive from $10 \%$ of span to $70 \%$ of span. Thus with respect to the averagepassage flow field, there exists an energy sink between $10 \%$ and $70 \%$ of span and an energy source outboard of $70 \%$ of span. The resulting redistribution of total temperature between Station 3 and 4 as a result of the radial derivative of this correlation is clearly evident. Outboard of $80 \%$ of span the time average total temperature at Station 4 is greater than that at Station 3, while between $10 \%$ of span and $80 \%$ of span the time average total temperature at Station 4 is less than that at Station 3.

In a similar fashion it may be shown that the correlations formed by the radial component of $\tilde{u}_{i_{(n)}}$ and the vector $\tilde{u}_{i_{(n)}}$. account for the spanwise redistribution of momentum with respect to the average-passage flow field attributed to the 
unstcady deterministic flow state.

As shown by Alkins and Smith (1982), accounting for spanwise redistribution of total temperature and momentum attributed to the unsteady deterministic flow state is very important in predicting the aerodynamic performance of multistage axial flow compressors. Likewise Lewis (1993) showed that accounting for the spanwise redistribution of total temperature and momentum attributed to the unsteady deterministic flow field is very important in predicting the aerodynamic performance of axial flow multistage turbines.

\section{Circumferential redistribution of total enthalpy and momentum}

A topic closely related to spanwise total temperature redistribution is circumferential total temperature redistribution. However, unlike spanwise redistribution no evidence has come forth which suggests that circumferential redistribution of total temperature (or equivalently total enthalpy) impacts aerodynamic performance. What has been shown is that circumferential redistribution of total temperature leads to the formation of hot spots on the surface of a turbine blade which impacts turbine life.

Butler, et al. (1989), showed that circumferential redistribution of an incoming time varying total temperature field can substantially increase the time average temperature of the pressure surface of a turbine rotor. Based on their numerical simulations, temperature spikes of $50 \mathrm{~K}$ above a background temperature of $388 \mathrm{~K}$ would increase the time average temperature of the pressure surface of a turbine rotor by $38.8 \mathrm{~K}$. Graham (1979), stated that an increase in metal temperature of $16.6 \mathrm{~K}$ above design intent can reduce the life of a turbine rotor by a factor of two. Thus even though the increase in pressure side temperature might appear to be small relative to the temperature of the incoming flow, it is large enough to significantly affect the life of a turbine rotor.

Sharma, et al. (1992) referred to the circumferential redistribution process of converting an unsteady total temperature field into a time average total temperature field as total temperature segregation. The fluid mechanic processes involved with total temperature segregation were first explained by Kerrebrock and Mikolajczak (1970) with respect to high-speed compressors. They observed that the absolute total temperature in the wake of a compressor rotor was higher than that outside of the wake as shown in Fig. (4). As the wake passed through the downstream stator, the wake particles drifted towards the stator pressure surface carrying with them an excess of total temperature. This redistribution of total temperature resulted in a circumferential variation in the time-average total temperature distribution exiting the stator. The total temperature was found to be higher adjacent to the pressure surface than it was near the suction side. In the case of a turbine, when the total temperature excess is the result of a hot streak formed in the rotor, the fluid particles within the hot streak drift toward the pressure surface of the downstream vane. The flow kinematics are shown in Fig. (5). In the frame of reference of the rotor, the fluid particles in the hot streak have the same flow direction as those of the cold fluid particles in the freestream. However, the magnitude of the relative velocity of the fluid particles in the hot streak is less than that of cold fluid particles in the freestream. In the frame of reference of the downstream vane, this difference in the magnitude of relative velocities leads to a drift of the fluid particles in the hot streak towards the pressure surface of the vane. The result is a significant increase in the time-average temperature of the vane pressure surface.

Total temperature segregation within the frame work of the average-passage model is accounted for by the averagepassage enthalpy flux associated with the unsteady deterministic flow field. The flow physics as modeling by Kerrebrock and Mikolajczak (1970) is accounted for by the first two terms in Eqn. (6). To show this consider the flow through a vane in which the incoming flow is unsteady in time. Once again the flow field is assumed to be deterministic, inviscid and adiabatic. The incoming unsteady flow is generated by an incoming nonuniform total temperature field (i.e. a hot streak). The incoming total pressure field is assumed to be independent of time and uniform in the radial and circumferential direction. The working fluid is an ideal gas with constant thermodynamic properties. The energy equation for this unsteady flow is Eqn. (8), while the equation governing the time average flow is Eqn. (16).

With respect to a control volume (i.e. see Fig. (6)) located away from either endwall and adjacent to the pressure surface of a vane, whose boundaries are defined by the streamlines associated with the time-average flow, Eqn. (16) may be rewritten as:

$$
\left.\bar{\rho}^{t}{\widetilde{T_{0}}}^{t} \widetilde{U}^{t} \delta\right|_{b}-\left.\bar{\rho}^{t}{\widetilde{T_{0}}}^{t} \widetilde{U}^{t} \delta\right|_{a}=-\int \overline{\rho T_{0 d} \overrightarrow{u^{\prime \prime}} \cdot \vec{n}} d s
$$

whose differential form is:

$$
\frac{d}{d s} \bar{\rho}^{t}{\widetilde{T_{0}}}^{t} \widetilde{U}^{t} \delta=-\left.\overline{\rho T_{0 d} \vec{u}^{\prime \prime} \cdot \vec{n}}\right|_{a} ^{b}
$$

where $\delta$ is the width of the control volume, $s$ is the distance along the pressure surface, $\widetilde{U}^{t}$ is the axial velocity and $\vec{n}$ is a unit vector normal to the control volume. Eqn. (17) is essentially the result derived by Kerrebrock and Mikolajczak (1970). At the surface of the vane $-\overline{\rho T_{0 d} \vec{u} \cdot \vec{n}}$ is zero. At the surface of the control volume labeled $a-b$ in Fig. (6), which is in the flow stream, the total temperature flux $-\overrightarrow{\rho T_{0 d} \overrightarrow{u^{\prime \prime}} \cdot \vec{n}}$ is positive. This indicates a flow 
of tot.al temperature into the control volume. This positive flux increases the time-average total temperature along the pressure surface. Along the suction surface the flux is negative which results in a reduction of the time-average total temperature along the suction surface.

As was previously stated, there is no published data which suggests that total temperature segregation impacts aerodynamic performance. Therefore introducing models to account for total temperature segregation in simulations where the objective is estimates of aerodynamic performance is deemed unnecessary. If the objective of the simulation is an assessment of the impact of total temperature segregation on turbine blade surface temperature, there appears to be a need for inclusion of models which account for total temperature segregation in the simulation. However this is not necessary because it is possible to construct credible estimates of the segregated total temperature field from average-passage simulations which are devoid of the flow physics associated with segregation as part of a post processing procedure. This post processing procedure entails estimating the temporal correlation which appears in Eqn. (18) from results obtained from the average-passage flow state of the incoming hot streak. With the temporal correlation known and assuming that the velocity in Eqn. (18) can be approximated by the average-passage velocity field of the blade row through which the hot streak is passing, Eqn. (18) may be solved to yield the segregated total temperature field produced by the incoming hot streak.

To test the validity of the above procedure for estimating hot streak segregation, Kirtley, et al. (1993) applied the procedure to the passage of a hot streak through a turbine stator. The hot streak was generated by the secondary flow field within the upstream rotor. Kirtley, et al. (1993) used a code which solved the average-passage flow equations and an unsteady wake-blade-row interaction code. Both codes assumed all metal surfaces to be adiabatic. The simulation based on the average-passage code did not incorporate a model for the segregation of the hot streak as it passed through the stator. In the unsteady simulation, the incoming unsteady flow to the stator was established from the average-passage simulation of the upstream rotor. Figure (7) shows a cross channel view (i.e axial plane) of the incoming total temperature distribution to the stator at an instant in time. The hot streak is located near $90 \%$ of span.

The average-passage simulation of the rotor (i.e. the average-passage flow field in which the incoming hot streak is defined) was used to estimate the temporal correlation which appears on the right-hand side of Eqn. (18). Using the velocity and density field associated with the averagepassage flow field of the stator, Eqn. (18) was solved to determine the time average total temperature distribution along the stator suction and pressure surface. The only cmpirical information used in the solution of Eqn. (18) was the axial location at which the correlation on the righthand side of Eqn. (18) became small. This information was deduced from the unsteady simulation and set at $50 \%$ of chord.

The chord-wise time-average temperature distributions along the suction and pressure surface at $90 \%$ of span from the average-passage simulation of the vane, the vane average-passage simulation as corrected by Eqn. (18), and the unsteady simulation are shown in Fig. (8). The temperature has been normalized with respect to the mass-average incoming total temperature. The time average surface temperature distribution derived from the unsteady simulation is shown as a solid line in the figure. The chord-wise variation of the time average surface temperature is a result of the vane secondary flow field and total temperature segregation. The average-passage simulation of the vane, devoid of the flow physics associated with segregation, only accounts for the redistribution of total temperature due to the vane secondary flow field. On the pressure side the temperature predicted by the average-passage simulation falls short of that predicted by the unsteady simulation. The average-passage result, as corrected by the procedure outlined above, agrees fairly well with that predicted by the unsteady simulation. The increase in temperature along the pressure surface from $20 \%$ of chord to near $50 \%$ of chord is captured by the correction procedure. In addition, the cooling of the suction surface as a result of the segregation process is also captured by the procedure.

An alternative procedure to account for total temperature hot streak segregation is outlined by Orkwis and Turner (1999).

Closely related to the topic of total temperature segregation is the flow process of circumferential transport of momentum attributed to the unsteady deterministic velocity field. All of the terms in Eqn. (5) play a role in the circumferential redistribution of momentum.

Both Valkov and Tan (1993), (1998) and Dawes (1994) examined the impact of this momentum transport on the performance of a compressor blade row. Dawes concluded that additional loss above that associated with mixing-out of the leakage flow was generated by the unsteady interaction involving the rotor tip leakage flow and the downstream stator. Unfortunately because of the procedure used by Valkov and Tan (1998) to estimate loss, the significance of their loss estimates is unclear. The significance and fluid mechanics responsible for loss generation in compressors due to unsteady interactions involving $2 \mathrm{D}$ wakes and blade boundary layers which are turbulent is unknown at this time.

Fritsch and Giles (1993) conducted an analytical and numerical study to assess the impact on performance resulting 
from the passage of a wake through a turbine cascade. From their simulations it appears that the loss within the turbine passige is increased by having the wake convect through the turbine as compared to having it mix-out prior to entering the turbine. Whether there is an unsteady interaction between the incoming wakes and the blade boundary layers which contributes in a measurable way to loss generation in turbines is unknown at the present time.

\section{Wake Recovery}

Another unsteady process whose impact on blade row performance has been studied both analytically and through numerical simulations is wake recovery. Wake recovery, first identified by Smith (1966), involves the reduction or amplification of the velocity deficit of a wake by a reversible unsteady process that reduces or increases the mixing loss associated with the wake. Adamczyk (1996) has shown that wake recovery is directly tied to the portion of the averagepassage stress, Eqn. (5), formed by the unsteady deterministic flow field. All of the terms which appear in Eqn. (5) contribute to the wake recovery process.

A simple example which illustrates the fluid mechanics associated with the wake recovery process was given by Smith (1966) and is repeated here. Figure (9) shows a wake entering and exiting a compressor and turbine blade row. The flow field is assumed to be inviscid, two-dimensional and incompressible. The flow state is deterministic. Evaluating the circulation, $\Gamma$, around a circuit whose boundary lies along the centerline of the wake and its edge yields:

$$
\Gamma=v_{d} l
$$

where $l$ is the length of the wake filaments bounded by the two stagnation stream lines and $v_{d}$ is the velocity deficit of the wake, Fig. (9). According to Kelvin's theorem, Batchelor (1967), the circulation associated with the incoming wake must remain constant as the wake convects through the cascade. Hence the wake circulation at the inlet to the cascade is equal to that at the exit. This equality yields the following expression for the ratio of the velocity deficit of the wake at the exit of the cascade to that at the inlet:

$$
\left.v_{d}\right|_{\text {exit }}=\frac{\left.v_{d} l\right|_{\text {inlet }}}{\left.l\right|_{\text {exit }}}
$$

If the length of a wake segment is increased as it passes through the cascade, as illustrated in the case of a compressor cascade, its velocity deficit is reduced, leading to a reduction in the wake mixing loss. If the length of a wake segment is reduced, as it is in the turbine illustration, its velocity deficit is increased, enhancing the wake mixing loss. This simple example by Smith (1966) based on kinematic arguments illustrates the recovery process in both compressors and turbines.

Valkov and Tan (1993) and Deregel and Tan (1996) conducted a number of very detailed numerical simulations which confirmed the kinematic model proposed by Smith (1966) for wake recovery in axial flow compressors.

Adamczyk (1996) used a perturbation analysis to link wake recovery to wake mixing loss and to the averagepassage stress associated with the unsteady deterministic flow field. Adamczyk's (1996) analysis like that of Smith's (1993) assumed the flow to be two-dimensional, and incompressible. If one neglects the effect of viscosity as the wake passes through the cascade, the loss reduction or increase due to the wake mixing out after it exits the cascade as opposed to mixing out prior to entering the cascade is given as:

$$
\chi_{\text {inlet }}-\chi_{\text {exit }}=K_{\text {inlet }}-K_{\text {exit }}
$$

where $\chi$ is the loss coefficient at the inlet and exit to the cascade, respectively. $K_{\text {inlet }}$ is the flux in kinetic energy of the incoming unsteady flow, and $K_{\text {exit }}$ is the flux in kinetic energy of the exiting unsteady flow.

Whether the wake mixing loss is reduced by having the wake pass through a cascade prior to being mixed-out is dependent on the reduction of the kinetic energy associated with the unsteady deterministic velocity field across a blade row. This kinetic energy is equal to one half the sum of the diagonal components (i.e. the trace) of the average-passage stress associated with the unsteady deterministic flow field. The sum of the diagonal components of the average- passage stress associated with the unsteady deterministic flow field is a measure of the magnitude of the velocity field associated with this flow field.

The link between the recovery process and the component of the average-passage stress associated with the unsteady deterministic flow field is established by means of a transport equation formed by the vector dot product of the unsteady deterministic velocity and the linearized inviscid momentum equation which it is assumed to satisfy. The time average of the resulting transport equation when integrated across a cascade yields:

$$
K_{\text {inlet }}-K_{\text {exit }}=\frac{1}{L} \int_{v o l} \frac{\overline{u_{i}^{\prime \prime} u_{j}^{\prime \prime}}}{2} \frac{\partial U_{i}^{a p}}{\partial x^{j}} d V o l
$$

where $L$ is the pitch of the cascade. The details can be found in Adamczyk (1996).

The integral on the right hand side of Eqn. (22) controls the growth of the wake deficit as it passes through the cascade. This growth is the result of the wake being strained by the time average velocity field within the cascade. If this . integral is positive, the wake deficit is reduced and there is a transfer of energy from the unsteady deterministic flow field 
to) the average-passage flow field. A reduction in the wake deficit learls to a reduction in the wake mixing loss. If this integral is negative, energy is transferred from the averagepassage flow field to the unsteady deterministic flow field. This transfer of energy increases the wake deficit leading to an increase in wake mixing loss.

Adamczyk (1996) noted that in many cases the transfer of energy from the unsteady deterministic flow field to the average-passage flow field is opposite to that which occurs between the nondeterministic flow field (turbulent flow state) and the average-passage flow field. For a turbulent flow, energy flows from the mean flow to the unsteady chaotic flow associated with turbulence where the energy cascades down to fine scale eddies and is dissipated, resulting in an increase in the internal energy (i.e. temperature) of the mean flow. The common practice of using a gradient diffusion model with a positive diffusion coefficient (i.e. positive eddy viscosity) to model this energy transfer process is well justified. However, the use of a gradient diffusion model to account for wake recovery may be questionable. In the case of wake recovery in compressors, the energy associated with the unsteady deterministic flow field of the wakes is reduced by the strain imposed on the wakes as they convect through a blade row. The energy loss by the unsteady deterministic flow field is transferred by a reversible process to the time average flow field. The use of a gradient diffusion model to account for wake recovery in compressors would thus require a negative diffusion coefficient, which is physically difficult to justify.

There is some data which shows the need to account for the wake recovery process in simulating multistage turbomachinery flows especially at off-design operating conditions. To model the recovery process within the context of the average-passage flow model, one must realize that recovery is accounted for through the portion of the average-passage stress associated with the unsteady deterministic flow field. Furthermore, it is the strain imposed on the wakes as they convect through a blade row and the subsequent modification of the average-passage stress which needs to be accounted for. The strain responsible for the wake recovery process can be decomposed into two components. The first component is associated with the axisymmetric flow field of the blade row through which the wake is passing. The other component is associated with the strain imposed by the time average non-axisymmetric flow field of the blade row through which the wake is passing. The strain imposed by the axisymmetric flow field is accounted for in the procedure outlined by Adamczyk, et al. (1986). The strain imposed by the time average non-axisymmetric How field is currently not being accounted for in simulations based on the average-passage flow model.

Recent works by Van Zante, et al. (1997) and van de
Wall (1999) provide models which account for the strain imposed by the time average non-axisymmetric flow field on recovery. However, these models remain to be incorporated into simulations of the average-passage flow field. The model developed by Van Zante, et al. (1997) extended the model of Smith (1993) to include the effects of viscosity. Van Zante's (1997) model provides an estimate of the kinetic energy associated with the unsteady deterministic velocity field of the wake as it convects through a blade row. The result of Van Zante's (1997) analysis was compared to LDV measurements from a single stage high-speed compressor. The agreement between the predicted decay of the velocity deficit of the rotor wake and that measured was quite good. Van Zante's (1997) results showed that the decay of the rotor wake as it convected through the stator was dominated by the kinematic recovery process as outlined by Smith (1966) and not by viscosity.

van de Wall (1999) was able to develop a transport model for the average-passage stress associated with the unsteady deterministic flow field. Van de Wall's model is three-dimensional and includes the effects of viscosity. By solving this transport equation van de Wall (1999) was able to estimate the decay of the kinetic energy associated with the unsteady deterministic flow field. For a compressor, van de Wall's (1999) findings were similar to those of Valkov and Tan (1998) and Van Zante, et al. (1997). All these researchers found that wake mixing loss in a compressor is reduced by nearly $70 \%$ as a result of the wake being strained by the velocity field associated with the averagepassage field of the blade row through which it is passing. At the design point of a well designed machine, the mixing loss of a compressor blade is estimated to be $15 \%$ of the total loss. The effect of recovery reduces the mixing loss to $4.5 \%$ of the total, a $10.5 \%$ reduction in the total loss. At an off-design operating condition of an axial compressor, where there might be significant regions of flow separation, the effect of recovery becomes even more important. Smith (1996) estimated that at the design point of a well-designed axial compressor recovery can yield a half point increase in efficiency.

For a turbine van de Wall (1999) found that recovery has a detrimental impact on performance. For an inviscid flow van de Wall (1999) showed that the velocity deficit within a stator wake increased as it passed through the downstream rotor. Viscosity was found to limit the growth of the velocity deficit, but because this process is non-reversible, it leads to an increase in the loss of total pressure across the blade row through which the wake is passing. The more the wake decays prior to entering the downstream blade row, the less the loss in total pressure across the blade row through which the wake is passing.

The recovery process can also lead to an increase in the 
pressure rise across a compressor blade row, and to a reduction in the pressure drop across a turbine blade row. The mathernatical details are given in Appendix B. According to Eqn. (B11) the pressure rise coefficient $C_{p}^{u s}$ resulting from an unsteady incoming vortical flow is:

$$
\begin{aligned}
C_{p}^{u s} & =\frac{\left.\overline{\vec{p}}^{y}\right|_{\infty}-\left.{\overline{\bar{p}^{t}}}^{y}\right|_{-\infty}}{\left.(1 / 2) q_{r e f}^{2}\right|_{-\infty}} \\
& =1-\frac{\cos ^{2} \beta_{-\infty}}{\cos ^{2} \beta_{\infty}} \\
& +\left[\left.\delta_{R}\right|_{-\infty}-\left.\delta_{R}\right|_{\infty}\left(\frac{\cos ^{2} \beta_{-\infty}}{\cos ^{2} \beta_{\infty}}\right)\right]
\end{aligned}
$$

where $\beta_{-\infty}$ and $\beta_{\infty}$ are the inlet and exit flow angles of the steady flow, respectively. The first two terms in Eqn. (B11) is the pressure rise produced by the steady incoming flow while the term in parentheses is that attributed to the incoming unsteady vortical flow. The variable $\delta_{R}$ is the recovery energy thickness defined by Eqn. (B10). This thickness is a measure of the flow blockage attributed to the unsteady vortical flow. Its relationship to the portion of the average-passage stress associated with the unsteady deterministic flow field is given by Eqn. (B20).

For a compressor cascade, $\cos ^{2} \beta_{-\infty}<\cos ^{2} \beta_{\infty}$, thus the incoming unsteady flow will increase the pressure rise across the cascade if $\left.\delta_{R}\right|_{-\infty}>\left.\delta_{R}\right|_{\infty}\left(\frac{\cos ^{2} \beta_{-\infty}}{\cos ^{2} \beta_{\infty}}\right)$. For a turbine cascade, $\cos ^{2} \beta_{-\infty}>\cos ^{2} \beta_{\infty}$, and the incoming unsteady flow will increase the pressure drop across the cascade if $\left.\delta_{R}\right|_{-\infty}<\left.\delta_{R}\right|_{\infty}\left(\frac{\cos ^{2} \beta_{-\infty}}{\cos ^{2} \beta_{\infty}}\right)$.

To provide a quantitative estimate of the impact of incoming wakes on cascade pressure rise, consider first a compressor cascade which turns the flow through $25^{\circ}$. The incoming flow is at an angle of $46^{\circ}$ with respect to the axial direction. The flow exits the cascade at $21^{\circ}$. The flow conditions for this compressor and its geometry are those used by Valkov and $\operatorname{Tan}$ (1998) in their study. The incoming wakes are one-dimensional shear flows which are assumed to be perpendicular to the incoming flow. As the wakes convect through the cascade, they are strained by the velocity field within the cascade passage. This straining was shown to reduce the wake velocity deficit. This reduction in the wake velocity deficit corresponds to a reduction in the kinetic energy of the unsteady flow associated with the wakes. For this cascade the reduction in the kinetic energy of the unsteady flow-field across the cascade is approximately $61 \%$. The reduction of the magnitude of the unsteady axial velocity component across the cascade is approximately $28.5 \%$. These values are extracted from the kinematic model put forth by Smith (1993) for wake recovery. Based on these values, the pressure rise across the cascade attributed to the steady potential flow according to Eqn. (B11) is:

$$
C_{p}^{(0)}=0.446
$$

while that attributed to the unsteady flow (i.e. $C_{p}^{(2)}=$ $\left.C_{p}^{u s}-C_{p}^{(0)}\right)$ is approximately:

$$
C_{p}^{(2)}=\left.(0.071) \frac{\overline{\vec{u}^{(1)} \cdot \vec{u}^{(1)}}}{\vec{u}^{(0)} \cdot \vec{u}^{(0)}}\right|_{-\infty}
$$

Equation (24) shows that the pressure rise attributed to the unsteady flow is proportional to the velocity deficit of the incoming wake squared. Assuming the unsteady flow is generated by wakes whose deficit is $25 \%$ of the incoming flow, the increase in pressure rise attributed to the incoming wakes is approximately $1.0 \%$. For this cascade Valkov and Tan (1998) reported an increase in static pressure rise of only .5\%. Valkov and Tan's (1998) estimate was deduced from a numerical simulation which included the effects of viscosity. Whether the factor of two difference in the estimate of the benefit of recovery is solely related to the effects of viscosity is unclear.

Smith (1969) reported the pressure rise per stage of a four stage compressor was increased by approximately $4 \%$ as the axial gap between the blade rows was reduced. The midspan geometry of the compressor reported upon by Smith (1969) is very similar to the cascade geometry described above. If one assumed this pressure rise was solely due to the recovery process and that the pressure rise is equally split between the rotor and the stator, the $4 \%$ per stage is equal to $2 \%$ per blade row. The current analysis and that of Valkov and $\operatorname{Tan}$ (1998) indicates that the increase in pressure rise resulting from incoming wakes should scale as the relative wake deficit squared (i.e. wake deficit relative to the incoming relative flow). Using Smith's (1969) data and assuming the wake deficit in the compressor analyzed by Smith (1969) is $25 \%$ of the incoming flow at the nominal axial spacing and $50 \%$ at half the nominal spacing (i.e., estimates from Valkov and Tan (1998)) the increase in pressure rise across a single blade row attributed to incoming wakes is:

$$
C_{p}^{(2)}=\left.(0.0475) \frac{\overline{\vec{u}}^{(1)} \cdot \vec{u}^{(1)}}{\vec{u}^{(0)} \cdot \vec{u}^{(0)}}\right|_{-\infty}
$$

For a wake deficit of $25 \%$ of the incoming flow, the increase in pressure rise according to Eqn. (25) is nearly .7\%. This number is slightly greater than that given by Valkov and Tan (1998) and less than that given by the current analysis.

In the above example had the compressor cascade turned the flow to axial, which would imply a flow turning of $46^{\circ}$ 
(a value representative of arlvanced designs) the increase in pressure rise as estimated by Eqn (B11) would be:

$$
C_{p}^{(2)}=\left.(0.1) \frac{{\overline{\vec{u}^{(1)} \cdot \vec{u}^{(1)}}}^{t}}{\vec{u}^{(0)} \cdot \vec{u}^{(0)}}\right|_{-\infty}
$$

For this cascade the pressure rise produced by the steady flow according to Eqn. (B11) is approximately:

$$
C_{p}^{(0)}=0.5
$$

For a wake deficit of $25 \%$ of the incoming flow, the increase in pressure rise is $1.25 \%$ of that produced by the steady flow. If the pressure rise increase were equally split between a rotor and stator, the increase in stage pressure would be $2.5 \%$, a number which is not all that insignifcant. One may view this number as an upper bound whose value is reduced because of viscosity. The magnitude of the impact of viscosity on the wake recovery processes is not clear.

The significance of the recovery process on aerodynamic performance depends on the design parameters of a machine. As the aerodynamic loading of blade rows is increased in an attempt to design machinery with fewer parts and of reduced weight, the blade wakes will deepen and the recovery process will have to be accounted for. Recovery is also more important at off-design conditions, where large regions of flow separation generate large velocity deficits.

\section{Blade Indexing.}

This section is concluded with a brief discussion of the components of the average-passage stress and the averagepassage enthalpy flux that is associated with the blade rows which are in the same spatial frame of reference. They are the last terms in Eqn. (A9) and (A10). These components of the average-passage stress and the average- passage enthalpy flux account for the effect of circumferential spatial distortions with length scales unrelated to the pitch of the blade row of interest. These components account for performance changes brought about by circumferential indexing or clocking of blades which are in the same reference frame. The experimental findings of Barankiewicz and Hathaway (1997) suggest that the effect of indexing in axial flow compressors is small and therefore need not be addressed. However for turbines, the work of Ardnt (1991) and Sharma, et al. (1997) shows that there are performance gains to be had through the indexing of blade rows.

The fact that indexing has a measurable impact on turbine performance and an insignificant impact on compressor performance is an indication the turbine wakes persists far longer than compressor wakes. This observation is consistent with the findings of van de Wall (1999).
Stress and total enthalpy flux models which account for the effect of indexing on the average-passage flow field have yct to be developed. However, the procedure outlined by Adamczyk, et al. (1986) may be used to estimate the magnitude of the third term in Eqn. (A9) and (A10) attributed to the average-passage flow fields other than that of the blade row of interest. The resulting estimate accounts for the straining and rotation of wakes by the axisymmetric flow field and the vorticity generated by this interaction.

\section{Examples of Simulations}

The previous section presented a number of examples which illustrate the effects of the unsteady deterministic flow field on the average-passage flow field and how these effects may be accounted for in CFD simulations of multistage axial flow turbomachines. In this section, the results from a number of simulations will be presented to illustrate the predictive capabilities afforded by incorporating these models into a CFD code. The code used to generate these results is referred to as APNASA. Some of the results to be presented are predictions and will be noted as such. A predictive result implies the simulation was done prior to the experiment. Some of the simulations were done by researchers in engine companies who are attempting to assess the predictive capabilities of this code. In all cases no information was provided to APNASA which set the aerodynamic matching of stages. The aerodynamic matching of stages and the flow blockage associated with that matching is an output of the simulation and not an input.

\section{Low Speed Research Compressor}

Figure (10) shows the measured pressure rise characteristic along with simulation results at a flow coefficient of 0.395 and 0.375 for the Lewis low speed axial compressor (LSAC). LSAC is a four stage machine with an inlet IGV, Wellborn and Okiishi, (1996), which is representative of the rear stages of a high pressure (H.P.) compressor. The compressor is of a modern design employing hub-shrouded stators with end-bends. The four stages are geometrically identical. The simulation accounted for the rotor tip clearance. The simulation did not include the stator hub cavities nor did the simulations account for stator hub leakage. Two experimental results are shown one for a baseline leakage with stator hub cavities present, the other with no leakage and no cavities present.

At the flow coefficient of 0.395 (which is near the measured peak efficiency operating point) and at the flow coefficient of 0.375 , the simulation results are in good agreement with the measurement. An attempt to simulate an operating condition near peak pressure failed to converge. The simulation did not account for casing treatment over . the first rotor which is present in the experiment. Tests with the casing treatment removed show that the compres- 
sor stalls at a flow coefficient near the peak pressure point of the characteristic.

Figure (11) shows the measured static pressure rise characteristic for each stage along with results from the simulations. The agreement between the simulation results and the datia is very good. For the flow coefficient of 0.395 , Fig. (12) shows plots of the total and static pressure coefficient, the axial and absolute tangential velocity, and the absolute and relative flow angle as a function of span for the simulation and the experiment. The plots are for an axial location behind the second stator. Once again, the agreement between the simulation results and the data is good. The slight difference between the static pressure coefficient derived from the simulation and that measured inboard of $40 \%$ span is unknown. For the same flow coefficient, Fig. (13) compares the simulated and measured results for an axial location behind the third stage rotor. The agreement between the simulation results and that derived from the measurements is comparable to that shown in Fig. (12). Additional comparisons are presented in Adamczyk, et al. (1998).

These results clearly show that the APNASA code with its current models that account for the effects of the unsteady deterministic flow field is, to a large extent, capturing the flow features which are setting the performance of the LSAC compressor.

\section{High Speed Ten Stage Compressor}

The next set of results are for the high pressure (H.P.) compressor of the GE 90 engine series. This compressor has ten stages plus an IGV. At the design point the first three stages of this compressor are tranisonic. The origin of this compressor dates back to the GE $E^{3}$ compressor, Wisler, (1977). In simulating this compressor, all known leakage and bleed flows were accounted for. The first set of results, Fig. (14), shows the total temperature and total pressure at the exit of each rotor as predicted by APNASA relative to that predicted by a refined quasi-three-dimensional flow code, CAFMIX II, developed by Smith (1999). The results are presented in terms of a relative difference between the APNASA predictions and those of CAFMIX II. The leakage and bleed flows are the same in both simulations. The simulated operating point of the compressor is near its design point. Figure (14) shows that the results from both models are in good agreement with each other throughout the compressor. The maximum difference in total temperature is less than $.8 \%$, and the difference in total pressure is less than $4 \%$. Figure (15) shows spanwise profiles of the normalized total pressure distribution and the normalized total temperature distribution at three axial locations within the compressor. The locations are the exit of the third and seventh stage and at the compressor discharge. Once again the agreement between the two mod- els is quite good. The average-passage model appears to give results which are very comparable to those of a refined quasi-three-dimensional flow model without the empiricism built into the quasi-three-dimensional model.

An interesting outcome of this study is a comparison between the flow blockage estimates of CAFMIX II and those deduced from the APNASA simulation, Turner (1999). The flow blockage deduced from the APNASA simulation is based on the definition of flow blockage used in the CAFMIX II code. The comparison is shown in Fig. (16). Even though the through-flow results from both codes are nearly the same, the flow blockage estimates are markedly different. For example, for the fifth stage the flow blockage estimate of CAFMIX II is a factor of two times larger than that deduced from the APNASA simulation. In addition, the flow blockage deduced from the APNASA simulation is almost constant throughout the compressor, while the estimate from CAFMIX II increases from the front to the discharge of the compressor. The CAFMIX II flow blockage estimate at the exit of the tenth stage rotor is more than twice that at the exit of the first stage rotor.

Since the predicted total temperature and total pressure rise through the compressor by both simulations was nearly the same, one may wonder if the difference in estimated flow blockage is being compensated for by differences in estimates of the flow angle exiting the blade rows. Figure (17) shows the spanwise distribution of the flow angle exiting the third stage rotor, the third stage stator, the sixth stage rotor, and the sixth stage stator as predicted by both codes. The agreement between the two predictions is good, but there are differences. The sensitivity of total pressure rise or total temperature rise to changes in flow blockage or exit flow angle for this compressor is unknown to the author. Whether the difference in the predicted exit flows seen in Fig. (17) compensates for the difference in estimated flow blockage seen in Fig. (16) is unknown.

The results presented in Figs. (16) and (17) raise questions as to how best to incorporate the results from a threedimensional simulation into a through-flow model. If the estimates of flow blockage derived from the APNASA simulation were introduced into CAFMIX II, it is speculated that the outcome would be an increase in the pressure rise across the aft stages, and a decrease in the pressure rise across the front stages for a fixed overall compressor pressure ratio. This would drive the agreement between the two models apart.

Unfortunately the compressor was never tested at the IGV setting, vane settings, and bleed rates corresponding to the APNASA and CAFMIX II simulations. Therefore, no true prediction of the compressor performance is available by which to judge either code. A series of simulations were executed using APNASA with the IGV and vanes set 
to their test settings in an attempt to match experimental mcasurements at a point on the operating line near design wheol speed. Additional simulations were executed at this whel speed in which the compressor was throttled from the operation line to near stall. The first set of results, Fig. (18) shows the relative difference in total pressure between the simulation result and the data at each stage in the compressor. The data was obtained from instrumentation mounted to the leading edge of the stators. The axial location of the simulation results is also the leading edge of stators and the compressor discharge. The agreement between the simulation results and the data is quite good. Figure (19) shows the spanwise distribution of normalized total pressure and total temperature at the leading edge of the third stage stator, the leading edge of the seventh stage stator and at the discharge of the compressor. Both simulation results and data are shown. The profiles resulting from the simulation are in good agreement with that inferred from the data, especially the total temperature profiles. The compressor efficiency as estimated by the simulation agreed very well with the measured efficiency.

The level of agreement shown in Figs. (18) and (19) required an adjustment of the bleed rates from the initial values specified. The initial values were best estimates prior to compressor tests. The final estimates were derived from measurements and a series of data match computations. Figure (20) shows the relative difference in total pressure at each stage based on the simulation used to generate the results in Fig. (18) and (19) (i.e., best estimate of bleed rates, IGV and vane settings) and two other bleed rate schedules. The first of these bleed rate schedules, annotated by shaded bars, corresponds to that used to generate the results in Figs. (14) through (17). For this bleed schedule the front end of the compressor becomes unloaded relative to the back end. The next result, annotated by open bars, was generated by lowering the third stage bleed rate to that measured. By drawing less third stage bleed air the predicted pressure ratio of the front stages increased to near their measured values, while that of the back stages was reduced. Finally, reducing the amount of bleed air being drawn from the seventh stage bleed to that measured lowered the predicted pressure ratio of stages eight through ten to near that measured. Stages one through four remained unchanged as stage seven bleed was reduced, while stages five through seven experienced an increase in pressure ratio. The results shown in Fig. (20) are quite significant for they clearly show how bleed can affect the matching of stages within a compressor. The initial simulation using the a priori estimates of bleed rates was judged to be less than satisfactory for design purposes. Clearly, in addition to having sound models to account for the unsteady flow field within axial flow multistage compressors, it is equally important to have credible estimates of the leakage flow rates and the bleed flow rates.

A series of simulations were also performed to ascertain APNASA's ability to compute the impact of throttling on compressor performance. The wheel speed for these simulations corresponds to that used in the previous simulations. Figure (21) shows the percent difference in total pressure ratio of individual stages relative to their predicted total pressure ratio at the operating line (i.e., simulation results used to generate the results in Fig. (18)). The pressure ratio being defined from stator leading edge to rotor trailing edge. For the tenth stage the pressure ratio is from the leading edge of the ninth stage stator to compressor discharge.

A series of simulations were executed for increasing back pressure until the code failed to converge. The highest total pressure ratio for which a converged solution was obtained is less than the total pressure ratio at which the compressor stalled. The first case labeled Case 1 is for a back pressure slightly greater than the value corresponding to Fig. (18), while Case 4 is for a back pressure setting very close to the numerical stall point. The other two cases are for back pressure settings which lay between these two extremes.

For Case 1, the slight increase in back pressure caused the last six stages to move slightly up their pressure characteristic. The operating point of the first four stages remains unchanged. Case 2 increased the back pressure further and caused all of the stages with the exception of stage 1 to respond. The mass flow, being set by the first stage, is unaffected by this increase in back pressure. The change in the pressure ratio of stages five through nine is significantly greater than that for stage ten. Stage ten show a modest change in pressure ratio which implies that it is operating on the flat part of its characteristic. Raising the back pressure still higher, Case 3 , further increases the pressure ratio of stages three through nine. Stages one and two remain unchanged as does the mass flow, while the pressure ratio across stage 10 decreases. Stage ten is operating on the positive side of its pressure characteristic. Throttling the compressor to near its numerical stall point, Case 4, causes an increase in pressure ratio of stages two through eight. The pressure ratio for stage one remains unchanged as does the mass flow. The pressure ratio for stages nine and ten has decreased. Both nine and ten are now operating on the positive side of their characteristic. The decrease in stage ten's pressure ratio from its previous value is quite noticeable. It appears that the pressure characteristic for stage ten rolls over very abruptly after peak pressure. Obtaining a converged solution at still higher back pressures proved difficult because of the extreme sensitivity to back pressure setting. It is thought that stage eight is operating near peak pressure and that any slight increase in back 
pressure causes its operating point to shift to the positive side of its pressure characteristic. When this occurs, the compressor numerically stalls. At this wheel speed, there is experimental evidence which suggests that stall originates in the eighth stage, Liou (1999).

\section{High Speed Three Stage Compressor}

The next set of results are for a small high-speed three stage plus IGV axial flow compressor. The compressor was designed, tested and simulated by Allied Signal, Mansour (1999). The compressor is of a modern design, employing transonic rotors in all three stages. Although the simulations are not a prediction (the tests predate the simulations) they were executed by a researcher who is not a developer of APNASA but rather a user who is attempting to assess its predictive capabilities. The total pressure characteristics and the total temperature characteristics of the individual stages as derived from the simulations and measurements are shown in Fig. (22). The experimental results were obtained from stator leading edge instrumentation. For the first stage, the total pressure ratio as well as the total temperature ratio is from the inlet to the exit of the first stage rotor. For stage two, the pressure ratio as well as the total temperature ratio is from the inlet of the first stage stator to the exit of the second stage rotor. For the third stage, the total pressure ratio as well as the total temperature ratio is from the inlet of the second stage stator to the exit of the third stage rotor. The stage total pressure ratios as well as the stage total temperature ratios are plotted as a function of corrected flow exiting each of the rotors. The characteristics are for the design wheel speed. The error between the simulated results and experimental data is also shown on the figure.

Two throttle settings were simulated in an attempt to bracket the design pressure ratio of the compressor. In both the simulation and test, the first stage operating point remains fixed as the compressor is throttled. This result implies that the second stage rotor is choked. The corrected mass flows exiting the first stage rotor, as derived from the simulations are approximately $1.5 \%$ greater than that deduced from the experimental measurements. For the second stage, the difference in corrected mass flow is $1.4 \%$. For the third stage, the difference between the corrected mass flow deduced from the data and that from the simulation results is $.4 \%$. Overall the agreement is quite satisfactory.

Figure (23) shows the total pressure ratio and the total temperature ratio at the exit of the first stage rotor as a function of span. Percent differences are also shown on the figure. Only the results from one simulation and one experimental point are shown since the operating point of the first stage rotor remained unchanged as the compressor was throttled. With the exception of the region near $90 \%$ of span, the agreement between the total pressure pro- files derived from the simulation and the experiment data is very good. At $90 \%$ of span, the total pressure profiles differ by less than $3 \%$. The total temperature profiles are also in good agreement. The difference between the two total temperature profiles at midspan is less than $1.0 \%$. It is encouraging to see that the simulation shows nearly the same rise in total temperature as the experiment outboard of $70 \%$ of span.

Figure (24) shows the pressure ratio and the total temperature ratio at the exit of the third stage rotor as a function of span. The pressure ratio associated with the two simulations brackets the experimental pressure ratio. The shape of the total pressure profiles are in reasonable agreement with each other. At midspan, the two simulations differ by less than $5 \%$, while the experimental value differs from either simulation result by less than $3 \%$.

The shape of the total temperature profiles shown in Fig. (24) are also in reasonable agreement with each other. However, the spanwise average total temperature from the two simulations is less than that deduced from the experiment. The relative difference between the experiment and simulation point 1 is approximately $1.3 \%$.

The agreement between data and simulation was judged to be sufficient for the purposes of using APNASA to guide the aerodynamic design of this multistage axial flow compressor, Mansour (1999).

\section{High Speed High Pressure and Low Pressure Turbine}

The final simulation examples are of a high pressure turbine (HPT) and low pressure turbine (LPT). The HPT is a 3/4 scale model of the HPT from the GE engine family, while the LPT is a $1 / 2$ scale model of the LPT from the GE engine family. The HPT is a two stage machine while the LPT is a six stage machine. The simulations of both machines included the effect of cooling and purge flows as well as variable gas properties. The simulations were set up to match the conditions for which data was available. The details of the simulations are given in Turner, et al. (1999). Figure (25) shows the spanwise profiles of total pressure and total temperature exiting the second stage rotor of the HPT. The measurements are represented by solid squares, while the simulation results appear as a solid line. The relative difference between the measurements and the simulation results is also shown on the figure. The agreement between the simulation and the data is very good. A comparison of the overall one-dimensional performance parameters showed that the simulation was $2.5 \%$ high in mass flow, $0.4 \%$ high in total pressure ratio and $1.6 \%$ low in total temperature ratio. Figure (26) shows a similar set of plots for the LPT. The spanwise profiles are at the exit of the sixth stage rotor. The relative difference between the measurements and the simulation is shown on the figure. The 
agreement between the two is also very good. For the LPT st.ully, a comparison of the overall one-dimensional performarce parameters showed that the simulation was $2.5 \%$ low in mass flow, $0.3 \%$ high in total pressure ratio, and $3.5 \%$ low in total temperature ratio.

The machines whose simulation results were presented in this section cover a broad range of multistage axial flow turbomachinery configurations. The objective of this section was to illustrate to the turbomachinery design community what could be, and what could not be, predicted by a code based on the average-passage flow model with the current procedures to account for the effects of the unsteady flow environment within multistage turbomachines. All the simulations were executed without recourse to information supplied by external models or data which prescribed the aerodynamic matching of stages, this information being an output of the simulations and not an input. Being able to rcliably establish the aerodynamic matching of stages in a multistage configuration is critical to ensuring that advanced designs attain their aerodynamic goals in the first build.

In the next section the current state of the art for simulating multistage axial flow turbomachines in support of aerodynamic design using models which are uncoupled from through-flow models is summarized. Suggestions for future work are also presented.

\section{Summary and Suggested Future Work}

Before summarizing it is important to stress the need to have correct geometry and correct inflow and outflow boundary conditions before attempting any simulation. This detail cannot be emphasized enough as evidenced by the findings of Shabbir, et al. (1997), Escuret and Veysseyre, (1997), Wellborn, et al. (1999). It is important to know the geometry at the flow conditions being simulated. This includes the blade geometry as well as the hub and shroud geometry. Rotor tip clearance, stator hub clearance, variable geometry setting, and variable geometry button configuration must all be known. Blade fillet geometry and surface finish must also be known. All cavities which are opened to the primary flow path must be known. All leakage flows must be known including those associated with shrouded blading, bleed flows, purge flows and the cooling flows of cooled turbines. If any of these details (geometry, inflow and outflow boundary conditions) are unknown, it is important to establish the sensitivity of the simulation results to their assumed values.

Table I is a bullet chart which summarizes the fluid mechanics addressed in this paper. At the top of the list is spanwise transport of wake fluid particles which leads to a redistribution of total temperature, and momentum. Although not specifically addressed, the spanwise redistribu- tion of entropy is also implied.

Next on the list is circumferential transport of wake fluid particles which also leads to a redistribution of total temperature and momentum. The flow physics associated with this redistribution was shown to be equivalent to that associated with spanwise redistribution of total temperature. Although circumferential redistribution of total temperature does not appear to impact aerodynamic performance, it does lead to total temperature segregation resulting in the formation of hot spots. These hot spots have a significant impact on turbine blade life.

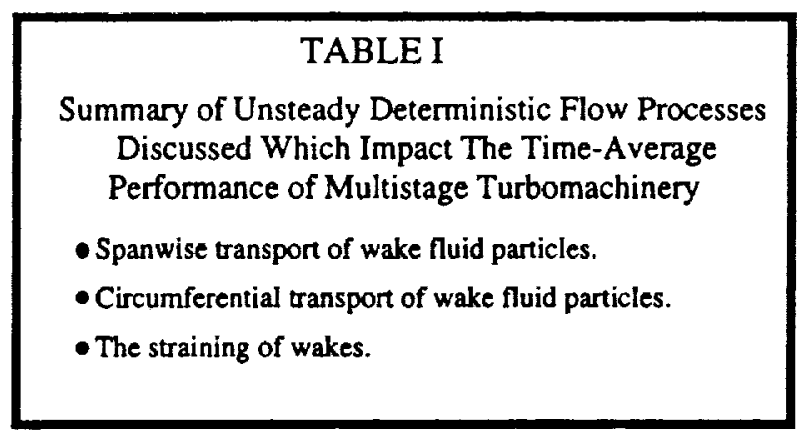

Circumferential redistribution of momentum also involves the interaction between a blade and incoming wakes and blade boundary layers. The impact of incoming wakes interacting with turbulent blade boundary layers on aerodynamic performance is not clear. There appears to be an indication that the interaction of compressor rotor tip clearance flows with a downstream stator leads to increased loss. However, when the interaction involves a transitional blade boundary layer, evidence exists that aerodynamic performance is impacted. This subject will be addressed later in this section.

Next on the chart is the straining of wakes as they convect through a blade row. This straining process leads to wake recovery. Wake recovery results in the transfer of energy by a reversible flow process between the unsteady flow generated by wakes and the time average flow field. Wake recovery was shown to impact the mixing loss of wakes as they pass through a downstream blade row. With respect to an axial flow compressor, the mixing loss attributed to 2D wakes is significantly reduced by the wake recovery process. For an axial flow turbine, the mixing loss attributed to 2D wakes is increased by the wake recovery process. Thus, there is a performance benefit to be gained in axial flow compressors by having blade rows closely spaced, while the opposite is true for axial flow turbines.

It was shown that the wake recovery process also impacted the pressure rise across the blade row through which the wakes are passing. The pressure rise was linked to flow blockage defined in terms of an energy recovery thickness. 
Sample results for an axial flow compressor showed that as the depth of wakes becomes large the pressure rise associat ed with the recovery process needs to be accounted for.

Models which attempt to account for the unsteady flow processes summarized above were used in generating the results presented in the Examples section. The results are encouraging, for they show that the average-passage flow model is capturing a good deal of the flow physics controlling the aerodynamic performance of axial flow multistage turbomachinery. However, there are a number of research studies which suggest the need to include additional models of unsteady flow phenomena. To list the key results from all these studies and to attempt to prioritize them in order of importance is beyond the scope of this work. However, there are a few topics which are on the author's short list for further study.

As two blade rows rotate past one another an unsteady flow field is generated. This unsteady flow field results in an unsteady aerodynamic force which acts on each blade row. When one blade row's unsteady force is viewed in the reference frame of the other its time average is nonzero. Thus, within the framework of the average-passage flow model the time average of both forces are accounted for by nonaxisymmetric body forces. With respect to the upstream blade row, the flow processes which produce the body force associated with the downstream blade row are similar to those encountered in the interaction of a total pressure distortion with a rotating blade row. The flow physics which produce the body force associated with the upstream blade row is similar to that involving a rotating blade row interacting with a downstream-generated flow distortion. The flow distortion is characterized in terms of a potential field. If the upstream blade row is a rotor, the non-axisymmetric body force associated with the rotor generates a circumferential variation in the total pressure and total temperature field. The generation of this total pressure and total temperature field and their interaction with the downstream stator was investigated by Shang, et al. (1993). Smith (1996) also reported experimentally observing a variation in the total pressure field downstream of a rotor embedded within a low speed multistage axial flow compressor. Smith (1996) speculated that if the region of high total pressure were to flow over the suction surface of the downstream stator a performance benefit would be realized.

Smith (1996) noted that the body force attributed to the downstream blade row results in a circumferential variation of the pressure field in the inter blade row region of a multistage axial flow compressor. This pressure field could reduce the pressure gradient along the suction surface of the upstream blade row. The reduced pressure gradient would lead to a reduction of the wake deficit and hence a reduction of wake mixing loss. Smith (1996) concluded that along with wake recovery the unsteady flow processes which lead to the development of the non-axisymmetric body forces contributed nearly equally to the measured efficiency gain of $1.5 \%$ brought about by reducing the axial gap between blade rows in a low speed 4 stage axial flow compressor. This is not an insignificant change and there is a need to model the fluid mechanics involved. A procedure for developing such models is presented by Giles (1992).

A number of experimental studies, Cumpsty, et al. (1995), Halstead, et al. (1997), Hodson (1990), and Walker (1974), have shown that flow transition is playing a role in establishing the aerodynamic performance of both axial flow compressors and low pressure turbines. The fluid mechanics involves the interaction between convecting turbulent wakes and transitional blade boundary layers. The impact of this process on the performance of low pressure turbines has recently been factored into their design, Harvey (1999).

The simulation of the NASA Lewis Low Speed Axial Compressor (LSAC) by Adamczyk, et al. (1998) clearly showed the need to account for transition in some form in order to match the measured performance. Figure (27) shows the results of a simulation performed using an averagepassage form of the Launder and Spalding (1974) $k-\epsilon$ model. Plotted is measured pressure rise of this compressor as a function of flow coefficient. The results from two simulations are also shown. The performance of this compressor as estimated by the simulations falls significantly short of that measured. The simulation was repeated with an improved $k-\epsilon$ model developed by Shih, et al. (1995) and the results are presented in Figs. (10) through (13). The improved model is not a transition model but it mimics the transition process. In the stagnation region and in regions where the pressure gradient is favorable, it suppresses the growth of the turbulent eddy viscosity. In regions where the pressure gradient is adverse, the turbulent eddy viscosity increases. For the LSAC, the Launder Spalding model produces a level of turbulent eddy viscosity along the suction surface of both rotors and stators typical of a high Reynolds number flow. At the stagnation region and along the suction surface where the pressure gradient is favorable the improved model of Shih produces a level of eddy viscosity which is slightly greater than the molecular viscosity. As flow diffuses along the suction surface the eddy viscosity grows to a level typical of a turbulent boundary layer. As previously noted the agreement of the improved model with data is very encouraging.

The improved model was also used to simulate an operating point of the GE 90 compressor. The results were only slightly different from a simulation executed using an average-passage form of the Launder and Spalding $k-\epsilon$. model. All of the previously reported simulations of the GE 90 and the Allied Signal compressor used the average- 
passage form of the Launder and Spalding $k-\epsilon$ model. The reason behind the small difference scen in the simulation of the GE 90 versus the rather large differences seen in the simulation of the LSAC is attributed to the difference in the blade Reynolds numbers and the blade surface finish of these two machines. When simulating machines whose blade Reynolds numbers are small, there is clearly a need to account for transition.

The final topic involves unsteady flows which are classified as nondeterministic but are not turbulent in nature. An example of this is the shedding of vorticies from a blade trailing edge, where the shedding frequency is not related to shaft rotational speed. Vortex shedding has been observed in fan rotors, Hathaway, et al. (1986) and has been shown to lead to spanwise redistribution of entropy, Kotidis and Epstein (1991). There is also an issue associated with the stability of shear flows in adverse pressure gradients. It has been observed that the stretching of vorticies leads to flow instabilities resulting in rapid mixing, Binder, et al. (1985) van de Wall, et al. (1995). A related subject is the stability. of rotor tip clearance flows. Based on experiments in a water tunnel, Zierke, et al. (1993) concluded that the tip vortex shed by a rotor meandered as it traveled downstream. Graf (1996) conducted a series of numerical simulations of a stage from a high-speed multistage compressor and observed a similar phenomena. These unsteady non-turbulent flows appear to lead to the mixing of shear layers and thus generate loss. The magnitude of the impact of such mixing processes on aerodynamic performance of multistage axial flow turbomachines is unknown. An effective means for modeling such mixing processes within the frame work of a time average flow model such as the average-passage flow model is not clear.

Finally there is a real need for executing well defined physical and computational experiments that focus on specific fluid mechanic issues related to the impact of unsteady flows on the performance of axial flow multistage turbomachines. Too often results are presented with no clear explanation as to their significance and the question they answer. Information such as this is not very useful in sorting out issues as complex as those involved in the fluid mechanics of multistage turbomachinery.

As computers become more powerful and the resolution capabilities of instrumentation improves, there is no doubt that many of the issues delineated in this section will be resolved. In addition, many new issues will appear that, at the present time, are unknown. One thing is certain, the modeling of multistage turbomachinery flow will remain a challenge for the foreseeable future and economic forces will continue to push the development of improved flow models for the purpose of supporting aerodynamic design.

\section{Acknowledgments}

The author wishes to thank the IGTI Scholar Lecture Committee and to those who have reviewed the drafts of this paper. The author wishes to express his gratitude to those managers, in industry and at NASA Lewis Research Center, who have supported the authors research endeavors. The author is also grateful to the many researchers in academia, industry and at NASA Lewis who have openly shared their research results and thoughts with the author on the fluid mechanics of multistage turbomachinery. Finally the author wishes to acknowledge the support and contributions of Mr. M. L. Celestina, Mr. R. A. Mulac, Mr. T. A. Beach, Dr. W. M. To, Dr. A. Shabbir, Mr. J. R. Wood, Dr. M. D. Hathaway and Dr. A. J. Strazisar to the development of the average-passage model and APNASA.

\section{Appendix A}

\section{A Three Dimensional Flow Model for Axial Flow Multi-Stage Turbomachines}

The procedure used for constructing the average-passage flow equations is based on the concept of mathematical filtering or averaging. Starting with the Navier-Stokes equations, the continuity equation, the energy equation and the equation of state, one can construct a sequence of mathematical filtering operations which result in a system of equations which govern the flow state resolved by the filtering operation. The resulting equations only resolve flow structures which are unaltered by the filtering operation. The resulting solution of the filtered equations is identical to that obtained by applying the filtering operation to the solution of the un-filtered equations. For example, the construction of the Reynolds-averaged form of the NavierStokes equation involves a filter which removes the nondeterministic flow structures associated with turbulence while retaining the deterministic structures. This is accomplished by using an ensemble-averaging filter. The resulting deterministic flow state as described by the Reynolds-averaged form of the Navier-Stokes equation is identical to that obtained by directly applying the ensemble-averaging filter to the solution of the Navier-Stokes equation.

The filtering operator used in the construction of the Reynolds-averaged form of the Navier-Stokes equations ensemble-averages consecutive samples of data taken over one shaft revolution. The mathematical form of the ensemble-averaging operator is:

$$
\begin{aligned}
\bar{f}^{e} & =\lim _{M \rightarrow \infty} \frac{1}{M} \sum_{m=1}^{M} f(r, \theta, z, \tau) \\
\tau & =t+\frac{2 \pi}{\Omega}(m-1)
\end{aligned}
$$




$$
t_{1} \leq t \leq \frac{2 \pi}{\Omega}+t_{1}=T+t_{1}
$$

where $m$, is an integer, $M$ is the number of shaft revolutions over which the data is collected, $f$, the data being sampled, $\bar{f}^{e}$ its ensemble average. The variable $\Omega$ is the shaft rotational speed, $t_{\mathrm{l}}$ is a reference time, and $\mathrm{T}$ is the time for one shaft revolution.

All flow features resolved by Eqn. (A1) are associated with the deterministic flow field. This includes all time dependent behavior with is directly linked to shaft rotational speed, $\Omega$. All flow features which are filtered out by Eqn. (A1) are associated with the non-deterministic flow field. Such features include turbulent eddies and shed vortices which are unrelated to shaft rotational speed. The global effect of the non-deterministic field on the deterministic field is accounted for by means of the Reynolds stress in the momentum equation and a total enthalpy flux in the energy equation. The Reynolds stress accounts for the transport of momentum between the deterministic and nondeterministic flow fields. These terms arise as a result of applying the filtering operator to the nonlinear terms which appear in the momentum and energy equations. The modeling of the Reynolds stress and the total enthalpy flux is the closure problem associated with solving turbulent flows. Equations for these terms must be provided and they form part of the equation system for the deterministic flow field.

As shown in Fig. (1) the Reynolds-averaged flow model has less fidelity than the Navier-Stokes equations. For the purpose of the present work, the Reynolds-averaged flow description of the deterministic flow field is time dependent.

To construct a time-average representation of the flow field within a multistage turbomachine requires the use of a time-average filtering operation. Two time-average flow fields can be defined: one with respect to the rotor frame of reference; and the other with respect to the stator or fixed frame of reference. For the stator frame of reference the time-average filter is defined by the following integral

$$
\bar{f}^{t}=\frac{1}{T \lambda} \int_{0}^{T} H_{G}(r, \theta, z, t) \vec{f}^{e}(r, \theta, z, t) d t
$$

where $H_{G}$ is a gate function whose value is one at an instant in time, $t$, when the axial position, $z$, the tangential position, $\theta$, and the radial position, $r$, define a point lying within the flow domain or on its boundaries. Otherwise the value of $H_{G}$ is zero. The variable $\lambda$ is defined as:

$$
\lambda \equiv \frac{1}{T} \int_{0}^{T} H_{G}(r, \theta, z, t) d t
$$

and is a measure of the metal blockage attributed to the rotors. For the rotor frame of reference, the filter is defined by replacing in both Eqns. (A2) and (A3), the absolute tangential position, $\theta$, with the relative tangential position, $\theta_{\text {rel }}$.

Equation (A2), applied to the deterministic form of the continuity equation, the momentum equation, the energy equation, the equation of state, and the closure equations associated with the Reynolds stress and the total enthalpy flux yields a set of equations which govern the time-average flow field in the fixed frame of reference. The resulting system of equations contain additional terms relative to those which appear in the deterministic equation system. This is again the direct result of the averaging operation. The continuity, momentum and energy equations all contain the metal blockage term $\lambda$. The momentum equation contains a body force and a stress tensor involving products of the unsteady deterministic velocity components. The form of this tensor is identical to that for the Reynolds stress and accounts for the transport of momentum between the deterministic flow field and the time-average flow field. The body force is the result of the time-averaged pressure and shear force imposed by the rotor blades on the fluid. The energy equation contains an energy source (sink) and an total enthalpy flux correlation. The energy source is associated with the work input attributed to the body force, while the total enthalpy flux accounts for the transfer of total enthalpy between the deterministic and time-average flow field.

The time-average flow model is also shown in Fig. (1), and because it is of lower fidelity for a multistage turbomachine application than the Reynolds-average model, it lies to its right. For the case of a single rotor or stator operating in a stable aero mode (i.e. free of rotating stall or surge), with uniform circumferential inflow and outflow boundary conditions, the time-average flow model and the Reynoldsaverage flow model are mathematically equivalent.

For a single stage configuration operating in a stable aero mode with uniform circumferential inflow and outflow boundary conditions, the time-average flow field for either the rotor or stator is periodic in the tangential direction with a period equal to the pitch of the respective blade row. Thus the time-average equation system for the rotor and stator is by definition the average-passage description for the stage as defined by Adamczyk (1985). However, with respect to the stator frame of reference, if additional stators are present whose blade count is different from that of the reference stator the resulting time average flow field will have a circumferential periodicity other than that of the reference stator.

Construction of an average-passage description of the flow field in a multistage machine therefore requires an additional averaging operation, Adamczyk (1985). For a given 
st.at,or this average operation is defined by the cquation:

$$
\bar{f}^{a p}=\frac{1}{\Lambda N} \sum_{n=0}^{N-1} G\left(r, \theta+\frac{2 \pi n}{N}, z\right) \bar{f}^{t}\left(r, \theta+\frac{2 \pi n}{N}, z\right)
$$

where $n$ is an integer and $N$ is the number of passages in the stator. The variable, $G$ is a function composed of the product of $L$ gate functions, $g_{l}$,

$$
G(r, \theta, z)=\Pi_{l=1}^{L} g_{l}(r, \theta, z)
$$

assigned to each blade row in the stator frame of reference. The gate function for the $l_{t h}$ stator, $g_{l}$, is equal to zero when $r, \theta$ and $z$ lie within the interior of the $l_{t h}$ stator, otherwise its value is one. Thus the variable $G$ is a function whose value is one when, $r, \theta$ and $z$ lie within the interior or on the boundaries of the time average flow field. Otherwise, the value of $G$ is zero.

For the $j_{t h}$ stator, $\Lambda$ is defined by the equation:

$$
\Lambda=\frac{1}{2 \pi} \int_{0}^{2 \pi} \Pi_{\substack{l=1 \\ l \neq j}}^{L} g_{l}(r, \theta, z) d \theta
$$

and is a measure of the metal blockage of the stator blade rows other than the one of interest. Each stator blade row has associated with it a filter as defined by Eqns. (A4), (A5) and (A6).

With respect to a given stator blade row, Eqn. (A4) forms the average-passage value of $\bar{f}^{t}$ by summing values of $G \bar{f}^{t}$ which are shifted tangentially by the pitch of the given stator. Hence by construction $\bar{f}^{a p}$ is periodic in the tangential direction with a period equal to that of the given stator blade row. $\bar{f}^{a p}$ so constructed is a discontinuous function, and this mathematical outcome of the averaging process described by Eqn. (A4) must be recognized.

A procedure similar to Eqn. (A4) is applied to the equations associated with the rotor time-average flow description. As in the case of each stator blade row, each rotor blade row is associated with a unique averaging operator. Hence each blade row in a multistage configuration has its own average-passage description which by construction is independent of time and periodic over the pitch of that blade row. The average-passage flow description is by construction the flow-field turbomachinery designers have heuristically thought of when executing an aero-design. The inlet and exit boundaries of each of these flow descriptions is at the physical inlet and exit of the machine.

Application of the filtering and averaging operations Eqns.(A1), (A2) and (A4) to the axial momentum equation associated with the fixed frame of reference yields:

$$
\frac{\partial}{\partial r}\left(\lambda^{a p} r \rho^{a p} V^{a p} U^{a p}\right)+\frac{\partial}{\partial \theta}\left(\lambda^{a p} \rho^{a p} W^{a p} U^{a p}\right)+
$$

$$
\begin{aligned}
\frac{\partial}{\partial z} \lambda^{a p}\left(r \rho^{a p} U^{a p} U^{a p}+p^{a p}\right) & =\frac{\partial}{\partial r} r \lambda^{a p}\left(\tau_{r z}^{a p}-R_{r z}\right) \\
& +\frac{\partial}{\partial \theta} \lambda^{a p}\left(\tau_{\theta z}^{a p}-R_{\theta z}\right) \\
& +\frac{\partial}{\partial r} r \lambda^{a p}\left(\tau_{z z}^{a p}-R_{z z}\right) \\
& +f_{z}^{(R)}+f_{z}^{(S)}
\end{aligned}
$$

where $\lambda^{a p}$ is the product of $\lambda$ and $\Lambda, p^{a p}$ is the pressure, $\rho^{a p}$ the density, $U^{a p}, V^{a p}$ and $W^{a p}$ the velocity components in the axial, radial and tangential direction respectively, and $\tau_{i j}^{a p}$ the viscous shear stress. The subscripts attached to $\tau_{i j}^{d p}$ identify the respective component. The superscript ap signifies that the variable is associated with the averagepassage flow state. The variable $f_{z}^{(R)}$ is the axial component of the body force generated by the rotor blade rows, while $f_{z}^{(S)}$ is the axial component of the body force generated by the stator blade rows other than the one of interest.

The body force whose axial component is $f_{z}^{(S)}$ is a singular function composed of Dirac delta functions, Lighthill (1964). Because of this singular behavior, the averagepassage flow variables are discontinuous at the location where this body force is singular. In dealing with this body force and the corresponding energy source (sink) only their non singular axisymmetric component is retained. The non-axisymmetric component (the singular portion) is omitted because it is non-physical, the singular behavior being strictly an outcome of the averaging operation Eqn. (A4). With the omission of the singular portion of the body force and energy source (sink), the average- passage flow variables are no longer discontinuous. They remain periodic in the tangential direction with a period equal to the stator of interest. In addition there still remains one unique axisymmetric flow state which is common to all the average-passage flow states.

In deriving Eqn. (A7) the following decomposition for the velocity field was used:

$$
\begin{aligned}
u_{i} & \equiv \widetilde{u_{i}^{e}}+u_{i}^{\prime}, \quad \widetilde{u_{i}^{e}}=0 \\
\widetilde{u_{i}} & \equiv \widetilde{\tilde{u}_{i}^{e}}+u_{i}^{\prime \prime}, \quad \widetilde{u_{i}^{\prime \prime}}=0 \\
\widetilde{u_{i}^{e}}{ }^{t} & \equiv U_{i}^{a p}+u_{i}^{\prime \prime \prime}, \quad \widetilde{u_{i}^{\prime \prime \prime}}=0, \quad U_{i}^{a p} \equiv \widetilde{{\widetilde{u_{i}^{e}}}^{a p}}
\end{aligned}
$$

where denotes a density weighted average, and the subscript $i$ (i.e. index notation) refers, respectively, to the axial, tangential and radial direction.

Equation (A7) also contains the axial component of a generalized Reynolds stress, $R_{i j}$, which will be referred to as the average-passage stress. It is defined as:

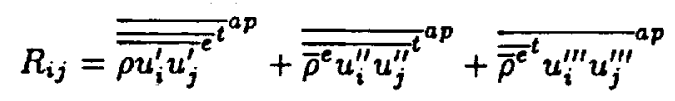


where the product, $\overline{\overline{\overline{\rho u_{i}^{\prime} u_{j}^{\prime}}}}{ }^{a p}$, is the contribution from the non-deterministic flow field, $\overline{\bar{\rho}^{e} \overline{u_{i}^{\prime \prime} u_{j}^{\prime \prime}}}{ }^{a p}$, the contribution

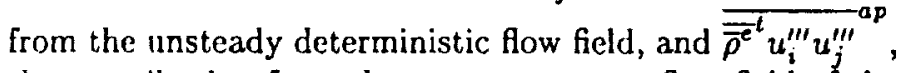
the contribution from the average-passage flow field of the stator blade rows other than the one of interest. The use of subscript $i$ and $j$ is standard tensor notation for orthogonal coordinate systems. The average-passage stress thus accounts for the effect of momentum transport associated with the non-deterministic flow field, the unsteady deterministic flow field, and the average-passage flow field other than that of the blade row of interest.

Application of the averaging operator in Eqn. (A4) to the energy equation associated with the time-average flow field in the fixed frame of reference results in an additional total enthalpy flux correlation which is associated with the average-passage flow field of the stator blade rows other than the one of interest. Taken together, the total enthalpy flux associated with the non-deterministic flow field, the unsteady deterministic flow field, and the average-passage flow field of stators other than the one of interest, they form the average-passage total enthalpy flux correlation:

$$
H F_{i}={\overline{\overline{\overline{\rho \bar{H}^{\prime} u_{i}^{c^{\prime}}}}}}^{a p}+{\overline{\overline{\bar{\rho}^{2} H_{o}^{\prime \prime} u_{i}^{\eta^{\prime \prime}}}}}^{a p}+{\overline{\overline{\bar{\rho}}^{t}} H_{o}^{\prime \prime \prime} u_{i}^{\prime \prime \prime}}^{a p}
$$

where $H$ is the total enthalpy. The decomposition of $H$ is identical to that of Eqn. (A8) for the velocity.

The averaging operator Eqn. (A4) is also applied to the equations used to estimate the Reynolds stress and total enthalpy flux associated with the non-deterministic flow field and the equations used to estimate their deterministic counterparts. As a result, additional terms may arise in the averaged form of these equations whose values must be estimated.

The closure terms associated with the average-passage equation system are the body force, energy source (sink), the average-passage stress, the average-passage total enthalpy flux, and any additional terms which arise from the application of the averaging operations to the equation used to estimate the Reynolds stress and the total enthalpy flux associated with the non-deterministic flow. Models are needed for the various closure terms which appear in the average-passage equation system, just as models for the Reynolds stress are needed in simulations of the deterministic flow field associated with a turbulent flow state. The development of these models is the closure problem associated with the average-passage equation system. The degree to which the closure models need to be developed is dependent upon the level of accuracy to which the averagepassage flow field is to be predicted.

\section{Appendix B}

\section{Pressure Rise Resulting From Wake Recovery}

The wake recovery process can impact the pressure rise across a cascade of airfoils. To show this consider the passage of two-dimensional wakes through a cascade of airfoils as shown in Fig. (9). The working fluid is assumed to be incompressible and inviscid. The flow field is assumed to be deterministic. The flow variables are non-dimensionalized with respect to the incoming fluid density (i.e., assumed to be constant), the axial chord length of the cascade, and the magnitude of the incoming flow velocity. The mechanical energy equation states that the time-average of the flux in total pressure entering the cascade must equal that exiting the cascade:

$$
\int_{\text {inlet }}{\overline{P_{0} u}}^{t} d l=\int_{e x i t}{\overline{P_{0} u}}^{t} d l
$$

where $P_{0}$ is the total pressure, $u$ the axial velocity component.

The total pressure is related to the pressure and the dynamic head by Bernoulli's equation:

$$
P_{0}=p+\frac{1}{2} \vec{u} \cdot \vec{u}
$$

Combining Eqns. (B1) and (B2), the time-average of the flux in total pressure becomes:

$$
\int{\overline{P_{0} u}}^{t} d l=\int \overline{p u}^{t} d l+\int \frac{1}{2}{\overline{u \vec{u} \cdot \bar{u}^{t}}}^{t} d l
$$

where the product $p u$ is the local pressure work, and $\frac{1}{2} \vec{u} \vec{u} \cdot \vec{u}^{t}$ is the flux of the kinetic energy of the flow stream.

Introducing Eqn. (B3) into Eqn. (B1) yields the following expression for the mass-average pressure rise or decrease across the cascade:

$$
\begin{aligned}
& \left.\overline{\bar{p}^{t}}{\overline{\bar{U}^{t}}}^{y} L\right|_{\infty}-\left.\overline{\widehat{p}^{y}}{\overline{\bar{U}^{t}}}^{y} L\right|_{-\infty} \\
& =\left.\int \frac{1}{2}{\bar{u} \vec{u} \cdot \bar{u}^{t}}^{t} d l\right|_{-\infty}-\left.\int \frac{1}{2} \overline{u \vec{u} \cdot \bar{u}^{t}} d l\right|_{\infty}(B 4)
\end{aligned}
$$

where $\widehat{\vec{p}}^{v}$, the mass-average pressure, is defined as

$$
{\overline{\bar{p}^{t}}}^{v}=\frac{1}{{\overline{\bar{U}^{t}}}_{L}^{y}} \int{\overline{p u^{t}}}^{t} d l
$$

and ${\overline{U^{t}}}^{y}$ is the time average axial velocity averaged over $\mathrm{L}$, the pitch of the cascade, Fig. (9):

$$
{\overline{\bar{U}^{l}}}^{y}=\frac{1}{L} \int \bar{u}^{t} d l
$$


At this point in the analysis, it is constructive to introduce the concept of flow blockage. In order to do so, a reference flow field must be defined. For this analysis, the reference flow field will be steady in time with a mass flow equal to that of the unsteady flow. The pressure rise across the cascade associated with this reference flow is,

$$
\begin{aligned}
& \left.p_{\text {ref } f}\right|_{\infty}{\overline{\bar{U}^{t}}}^{y} L-\left.p_{\text {ref }}\right|_{-\infty} \overline{\bar{U}}^{t} L \\
& =\left.\frac{1}{2} q_{\text {ref }}^{2}\right|_{-\infty}{\overline{\bar{U}^{t}}}^{y} L-\left.\frac{1}{2} q_{r e f}^{2}\right|_{\infty} \overline{\bar{U}}^{t}{ }^{y} L
\end{aligned}
$$

Subtracting Eqn. (B7) from Eqn. (B4) yields an equation for the increase or decrease in the pressure rise across the cascade attributed to the unsteady flow. This equation is:

$$
\begin{aligned}
& \left.\left({\overline{\bar{p}^{t}}}^{y}-p_{\text {ref }}\right)\right|_{\infty}{\overline{\bar{U}^{t}}}^{y} L-\left.\left({\overline{\vec{p}^{t}}}^{y}-p_{r e f}\right)\right|_{-\infty}{\overline{\bar{U}^{t}}}^{y} L \\
& =\left[\left.\frac{1}{2} \int \overline{u\left(\vec{u} \cdot \vec{u}-q_{r e f}^{2}\right)^{t}}\right|_{-\infty} d l\right] \\
& -\left[\left.\frac{1}{2} \int \overline{u\left(\vec{u} \cdot \vec{u}-q_{r e f}^{2}\right)}\right|_{\infty} d l\right]
\end{aligned}
$$

where $\bar{U}^{t}$ in Eqn. (B7) has been replaced by its integral form, Eqn. (B6).

Each of the bracketed terms on the right hand side of Eqn. (B8) may be rearranged into the following form:

$$
\begin{aligned}
& \frac{L}{2} \int \overline{u\left(\vec{u} \cdot \vec{u}-q_{r e f}^{2}\right)} \frac{d l}{L} \\
& =\frac{L}{2} q_{r e f}^{2}{\overline{\bar{U}^{t}}}^{y} \delta_{R}
\end{aligned}
$$

where the recovery energy thickness, $\delta_{R}$ is

$$
\delta_{R}={\overline{\frac{u}{\overline{\bar{U}}^{i}}\left[\frac{\overrightarrow{\vec{u} \cdot \vec{u}}}{q_{\text {ref }}^{2}}-1\right]}}_{t}^{t} \frac{d l}{\bar{L}}
$$

Note that the recovery energy thickness is similar in form to the energy thickness of boundary layer theory. If the flow is independent of time, $\delta_{R}$ is zero. Whether the recovery energy thickness is positive or negative depends upon the portion of the average-passage stress associated with the unsteady deterministic flow field.

Adding Eqn. (B8) to Eqn. (B7) with the bracketed terms in Eqn. (B8) replaced with Eqn. (B10) yields:

$$
\begin{aligned}
C_{p}^{u s} & =\frac{\left.\overline{\bar{p}^{\underline{y}}}\right|_{\infty}-\left.{\overline{\bar{p}^{t}}}^{v}\right|_{-\infty}}{\left.(1 / 2) q_{r e f}^{2}\right|_{-\infty}} \\
& =1-\frac{\cos ^{2} \beta_{-\infty}}{\cos ^{2} \beta_{\infty}} \\
& +\left[\left.\delta_{R}\right|_{-\infty}-\left.\delta_{R}\right|_{\infty}\left(\frac{\cos ^{2} \beta_{-\infty}}{\cos ^{2} \beta_{\infty}}\right)\right]
\end{aligned}
$$

where $\left.q_{\text {ref }}\right|_{\infty} /\left.q_{r e f}\right|_{-\infty}=\cos \beta_{-\infty} / \cos \beta_{\infty}$ with $\beta_{-\infty}$ and $\beta_{\infty}$ being the inlet and exit flow angles of the reference flow, respectively. $C_{p}^{u d}$ is the cascade pressure rise coefficient resulting from an unsteady incoming vortical flow. The first two terms in Eqn. (B11) is the pressure rise, $C_{p}^{(0)}$, produced by the steady incoming flow while the term in parentheses is that attributed to the incoming vortical flow.

Consider next a steady flow through a cascade in which the blockage at the inlet is $\left.\delta\right|_{-\infty}$ and that at the exit is $\left.\delta\right|_{\infty}$, Fig. (28). Within the core flow, the total pressure is uniform, hence

$$
\begin{aligned}
\left.P_{\circ}\right|_{\infty}=\left.P_{\circ}\right|_{-\infty} & =\left.p\right|_{\infty}+\left.\frac{1}{2} q^{2}\right|_{\infty} \\
& =\left.p\right|_{-\infty}+\left.\frac{1}{2} q^{2}\right|_{-\infty}
\end{aligned}
$$

The pressure rise produced by this steady flow across the cascade is,

$$
\left.p\right|_{\infty}-\left.p\right|_{-\infty}=\left.\frac{1}{2} q^{2}\right|_{-\infty}-\left.\frac{1}{2} q^{2}\right|_{\infty}
$$

At the inlet:

$$
\left.q\right|_{-\infty}=\left.\frac{{\overline{\bar{U}^{t}}}^{y} \sec \beta}{(1-\delta)}\right|_{-\infty}
$$

while at the exit,

$$
\left.q\right|_{\infty}=\left.\frac{\overline{\bar{U}^{t}} \sec \beta}{(1-\delta)}\right|_{\infty}
$$

Using Eqns. (B14) and (B15), Eqn. (B13) can be rewritten as a pressure rise coefficient,

$$
\begin{aligned}
C_{p} & =\frac{\left.p\right|_{\infty}-\left.p\right|_{-\infty}}{(1 / 2) \overline{\bar{U}}^{y} \sec \beta_{-\infty}} \\
& =1-\frac{\cos ^{2} \beta_{-\infty}}{\cos ^{2} \beta_{\infty}}+\left[\left.2 \delta\right|_{-\infty}-\left.2 \delta\right|_{\infty}\left(\frac{\cos ^{2} \beta_{-\infty}}{\cos ^{2} \beta_{\infty}}\right)\right](
\end{aligned}
$$

where $1 /(1-\delta)^{2}$ has been approximated by $1+2 \delta$ (i.e. $\delta<<1)$.

Comparing Eqn. (B11) to Eqn. (B16) shows that the pressure rise across a cascade due to an incoming unsteady vortical flow is equal to that produced by a steady flow in which the inlet flow blockage is $\left.(1 / 2) \delta_{R}\right|_{-\infty}$ while that at the exit is $\left.(1 / 2) \delta_{R}\right|_{\infty}$. Had the assumption not been made regarding the magnitude of $\delta$, the flow blockage of the equivalent steady flow would be:

$$
\delta=1-\frac{1}{\sqrt{1+\delta_{R}}}
$$


The link between the flow blockage attributed to the unstcady flow and the portion of the average-passage stress associated with the unsteady deterministic flow field is through the expression for the recovery energy thickness, Equ. (B10). To establish this relationship, it is assumed that the pressure and velocity field can be expanded in a perturbation series of the form

$$
\begin{aligned}
p & =p^{(0)}+\epsilon p^{(1)}+\epsilon^{2} p^{(2)}+O\left(\epsilon^{3}\right) \\
\vec{u} & =\vec{u}^{(0)}+\epsilon \vec{u}^{(1)}+\epsilon^{2} \vec{u}^{(2)}+O\left(\epsilon^{3}\right)
\end{aligned}
$$

The lowest order term in Eqns. (B18) and (B19) is associated with a steady potential flow. The first order pressure term is also associated with a potential flow field and thus vanishes far upstream and downstream of the cascade.

The time-average of the first order term in Eqns. (B18) and (B19) is zero. Flow continuity requires that the pitchwise average of the axial component of $\vec{u}^{(2)}$ be zero. Using the perturbation expansions given by Eqns. (B18) and (B19), the expression for the recovery energy thickness to second order is,

$$
\delta_{R}=\frac{\left.2 \epsilon^{2} k_{e}\right|_{-\infty}}{q^{(0)^{2}}}\left[\frac{\overline{v^{(0) v^{(2)} t^{t}}}}{\left.k_{e}\right|_{-\infty}}\right]
$$

\section{References}

Adamczyk, J.J., 1985, "Model Equation for Simulating Flows in Multistage Turbomachines," ASME paper 85-GT226.

Adamczyk, J.J., Mulac, R.A., and Celestina, M.L., 1986, "A Model for Closing the Inviscid Form of the AveragePassage Equation System," Journal of Turbomachinery, Vol. 108 p. 180.

Adamczyk, J.J., 1991, "A Mathematical Constraint Placed Upon Inter-Blade Row Boundary Conditions Used in The Simulation of Multistage Turbomachinery Flows," AGARD Conference Proceedings 510, CFD Techniques for Propulsion Applications.

Adamczyk, J.J., 1996, "Wake Mixing in Axial Flow Compressors," ASME paper 96-GT-029.

Adamczyk, J.J., Hathaway, M.D., Shabbir, A., and Wellborn, S.R., 1998, "Numerical Simulation of Multi-Stage Turbomachinery Flows," Presented at the Applied Vehicle Technology Symposium on Design Principles and Methods for Aircraft Gas Turbine Engines, hosted by AGARD in Toulouse, France May 11-15, 1998.

Adkins, G.G., Jr, and Smith, L.H., Jr, 1982, "Spanwise Mixing in Multistage Axial Flow Compressors," ASME Journal Engineering for Power, Vol. 104, p.97.

Arndt, N., 1991, "Blade Row Interaction In A Multistage Low-Pressure Turbine," ASME paper 91-GT-283.

Barankiewicz, W.S., and Hathaway, M.D., 1997, "Effects of Stator Indexing on Performance in a Low Speed

$$
\begin{aligned}
& +\frac{\left.2 \epsilon^{2} k_{e}\right|_{-\infty}}{q^{(0)^{2}}}\left[\frac{k_{e}}{\left.k_{e}\right|_{-\infty}}\right.
\end{aligned}
$$

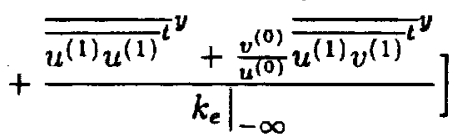

where

$$
k_{e}=\frac{1}{2}{\overline{\vec{u}^{(1)} \cdot \vec{u}^{(1)}}}^{r}
$$

and $q^{(0)}$ is the speed of the steady potential flow.

In order to simplify the process for estimating $\delta_{R}$ it is assumed that the exit flow angle defined in terms of the time average velocity field is unaffected by the incoming unsteady flow. This assumption implies that $\overline{v^{(2)}}$ at the exit of the cascade is zero.

Multistage Axial Compressor," ASME paper 97-GT-496.

Batchelor, G.K., 1967, "An Introduction to Fluid Dynamics," Cambridge University Press.

Binder, A., Foster, W., Kruse, H., and Rogge, H., 1985, "An Experimental Investigation Into the Effect of Wakes on the Unsteady Turbine Rotor Flow," ASME Journal of Engineering for Gas Turbines and Power, Vol. 107, p. 458.

Butler, T.L., Sharma, O.P., Joslyn, H.D., and Dring R.P., 1989, "Redistribution of an Inlet Temperature Distortion in an Axial Flow Turbine Stage," AIAA Journal of Propulsion and Power, Vol. 5.

Chen, J.P., Celestina, M.L., Adamczyk, J.J., 1994, "A New Procedure for Simulating Unsteady Flows Through Turbomachinery Blade Passages," ASME paper 94-GT-151.

Cumpsty, N.A., 1989, "Compressor Aerodynamics," Longman Scientific \& Technical, Publishers.

Cumpsty, N.A., Dong, Y., and Li, Y.S., 1995, "Compressor Blade Boundary Layers In The Presence of Wakes," ASME paper 95-GT-443.

Dawes, W.N., 1992, "Towards Improved Throughflow Capability," ASME Journal of Turbomachinery, Vol. 114.

Dawes, W.N., 1994, "A Numerical Study of the Interaction of a Transonic Compressor Rotor Over-tip Leakage Vortex with the Following Stator Blade Row," ASME paper 94-GT-156.

Denton, J.D., and Singh, U.K., 1979, "Time March- . ing Methods for Turbomachinery Flow Calculations," VKILEC-SER-1979-7, VKI. 
Denton, J., 1996, "Lessons Learned From Rotor 37," presented at the Third International Symposium on Experimental and Computational Aerothermodynamics of Internal Flows (ISAIF).

Deregel, P., and Tan, C.S., 1996, "Impact of Rotor Wakes on Steady State Performance of Compressor," ASME 96-GT-253.

Dorney, D.J., Davis, R.L., Edwards, D.E., and Madavan, N.K., 1990, "Unsteady Analysis of Hot Streak Migration in a Turbine Stage," AIAA paper 90-2354.

Escuret, J.F., and Veysseyre, P.H., 1997, "Effect of a Mismatch Between the Buttons of Variable Stator Vanes and the Flowpath in a Highly Loaded Transonic Compressor Stage," ASME paper 97-GT-471.

Fritsch, G., and Giles, M.B., 1993, "An Asymptotic Analysis of Mixing Loss," ASME paper 93-GT-345.

Gallimore, S.J., 1998, "Viscous Throughflow Modeling of Axial Compressor Bladerows Using a Tangential Blade Force Hypothesis," ASME Journal of Turbomachinery, Vol. 120, p.662.

Gallimore, S.J., 1998, "Axial Flow Compressor Design," The Successful Exploitation of CFD in Turbomachinery Design, IMechE,HQ,London.

Gallimore, S.J., 1999, Rolls Royce plc, Private Communication, Compressor Design

Giles, M.B., 1990, "Stator/Rotor Interactions in a Transonic Turbine," AIAA Journal of Propulsion and Power, Vol. 6, p. 621 .

Giles, M.B., 1992, "An Approach for Multi-stage Calculations Incorporating Unsteadiness," ASME paper 92-GT282.

Graf, M.B., Greitzer, E.M., Marble, F.E., and Sharma, O.P., 1999, "Effects of Stator Pressure Field on Upstream Rotor Performance," To be presented at the ASME IGTI Turbomachinery Conference 1999.

Graham, R.W., 1979, "Fundamental Mechanisms That Influence the Estimate of Heat Transfer to Gas Turbine Blades," ASME paper 79-GT-43.

Gundy-Burlet, K., 1993, "Computations of Unsteady Multistage Compressor with Swept Blades," ASME Journal of Turbomachinery, Vol. 115, No. 2.

Hall, E.J., and Delaney, R.A., 1993, "Investigation of Advanced Counter Rotation Blade Configuration Concepts for High Speed Turboprop Systems," NASA CR 187126.

Hall, E.J., 1997, "Aerodynamic Modeling of Multistage Compressor Flowfields," ASME papers 97-GT-344 and 97GT-345.

Halstead, D.E., Wisler, D.C., Okiishi, T.H., Walker, G.J., Hodson, H.P., and Shin, H., 1997, "Boundary Layer Development in Axial Compressors and Turbines Part 1 of 4: Composite Picture," Journal of Turbomachinery, Vol. 119 , p. 114.
Harvey, NW, Schulte, VS, Howell, RJ, and Hodson, HP, 1999.. "The Role of Research in the Aerodynamic Design of an Advanced Low Pressure Turbine ", 3rd European Conf. on Turbomachinery, IMechE, London, Mar.

Hathaway, M.D., Gertz, J.B., Epstein, A.H., and Strazisar A.J., 1986, "Rotor Wake Characteristics of a Transonic Axial-Flow Fan," AIAA Journal Vol. 24, No. 11, p. 1802.

Hodson, H.P. and Dawes, W.N., 1998, "On the Interpretation of Measured Profile Losses in Unsteady WakeTurbine Blade Interaction Studies," ASME Journal of Turbomachinery, Vol. 120, p. 276.

Hodson, H.P., 1990, "Modeling Unsteady Transition and Its Effects on Profile Loss," ASME Journal of Turbomachinery, Vol. 112, No. 4.

Howard, M.A., and Gallimore, S.J., 1993, "Viscous Throughflow Modeling for Multi-Stage Compressor Design," ASME Journal of Turbomachinery, Vol. 115, p.296.

Jennions, I.K., and Stow, P., 1986, "The Importance of Circumferential Non-Uniformities in a PassageAveraged Quasi-Three-Dimensional Turbomachinery Design System," ASME Journal Engineering for Gas Turbines and Power, Vol. 108.

Khalid, S.A., Khalsa, A.S., Waitz, I.A., Tan, C.S., Greitzer, E.M., Cumpsty, N.A., Adamczyk, J.J., and Marble, F.E., 1998, "Endwall Blockage in Axial Compressors," ASME paper 98-GT-188.

Kerrebrock, J.L., and Mikolajczak, A.A., 1970, "IntraStator Transport of Rotor Wakes and Its Effect on Compressor Performance," ASME Journal of Engineering and Power, p. 359.

Kirtley, K.R., Celestina, M.L., and Adamczyk, J.J., 1993, "The Effect of Unsteadiness on the Time-Mean Thermal Loads in a Turbine Stage," SAE paper 931375.

Kirtley, K.R., Turner, M.G., and Saeidi, S., 1999 , "An Average-Passage Closure Model for General Meshes," To be presented at the ASME IGTI Turbomachinery Conference 1999.

Kotidis, P.A., and Epstein, A.H., 1991, "Unsteady Radial Transport in Axial Compressor Stage," ASME Journal of Turbomachinery, Vol 113.

Launder, B.E., and Spalding, D.B., 1974, "The Numerical Computation of Turbulent Flows," Comp. Math. Appl. Mech.,3, p. 269.

LeJambre, C.R., Zacharias, R.M., Biederman, B.P., Gleixner, A.J., and Yetka, C.J., 1998, "Development and Application of a Multistage Navier-Stokes Flow Solver: Part II- Application to a High-Pressure Compressor Design," ASME Journal of Turbomachinery, Vol. 120, p. 215.

Lewis, K.L., 1993, "Spanwise Transport in Axial-Flow . Turbines," ASME paper 93-GT-289 and 93-GT-290.

Lighthill, M.J., 1964, "Introduction to Fourier Analy- 
sis and Generalized Functions," Cambridge Monographs on Mechanics and Applied Mathematics, Cambridge University Press.

Liou, G., 1999, GE Aircraft Engines, Private Communication, Compressor Stall.

Marble, F.E., 1964, "Three-Dimensional Flow in Turbomachines," High Speed Aerodynamics and Jet Propulsion Vol. X, Aerodynamics of Turbines and Compressors. Princeton University Press.

Mansour, M., 1999, AlliedSignal Engines, Private Communication, Multistage Compressor Simulations

Orkwis, P.D., and Turner, M.G., "An Eulerian/Lagrangian Approach for Analyzing Temperature Segregation Effects in High Pressure Turbines," To be published.

Rai, M.M., and Dring, R.P., 1987, "Navier-Stokes Analysis of the Redistribution of Inlet Temperature Distortion in a Turbine," AIAA paper 87-2146.

Reid, L., and Moore, R., 1978, "Design and Overall Performance of Four Highly Loaded, High-Speed Inlet Stages for an Advanced High-Pressure Ratio Core Compressor," NASA TP 1337.

Rhie, C.M., Gleixner, A.J., Spear, D.A., Fischberg, C.J., and Zacharias, R.M., 1998, "Development and Application of a Multistage Navier-Stokes Solver: Part 1 - Multistage Modeling Using Body Forces and Deterministic Stresses," ASME Journal of Turbomachinery, Vol. 120, p.205.

Serovy, G.K., 1981, "Axial-Flow Turbomachine ThroughFlow Calculation Methods," AGARD Advisory Report No. 175, Through Flow Calculations in Axial Turbomachines, Edited by Ch. Hirsch and J.D. Denton, 1981.

Shabbir, A., Celestina, M.L., Adamczyk, J.J., and Strazisar, A.J., 1997, "The Effect of Hub Leakage Flow on Two High Speed Axial Flow Compressor Rotors," ASME paper 97-GT-346.

Shang, T., Epstein, A.H., Giles, M.B., Sehra, A., 1993 , "Blade Row Interaction Effects on Compressor Measurements," AIAA Journal of Propulsion and Power, Vol. 9, No. 4, p. 569.

Sharma, O.P., Pickett, G.F., and Ni, R.H., 1992, "Assessment of Unsteady Flows in Turbines," ASME Journal of Turbomachinery, Vol. 114.

Sharma, O.P., Stetson, G.M., Daniels, W.A., Greitzer, E.D., Blair, M.F., and Dring, R.P., 1997, "Impact of Periodic Unsteadiness on Performance and Heat Load in Axial Flow Turbomachines," NASA Contractor Report 202319.

Shih, T.H., Liou, W.W., Shabbir, A., Zhu, J., and Yang, Z., 1995, "A New $k-\epsilon$ Eddy Viscosity Model for High Reynolds Number Turbulent Flows," Computers in Fluids, Vol 23, No. 3, p. 227.

Smith, L.H., Jr, 1966, "Wake Dispersion in Turbomachines," ASME Journal of Basic Engineering Vol. 88, Ser. D, No. 3 .
Smith, L.H., Jr, 1969, "Casing Boundary Layers in Multistage Compressors," Proceedings of the Symposium on Flow Research on Blading, Brown, Boveri \& Company, Baden, Switzerland Dzung, L.S., Editor, Elsevier Publishing Company.

Smith, L.H. Jr., 1993, "Wake Ingestion Propulsion Benefit," AIAA Journal of Propulsion and Power, Vol. 9, No. 1, p.74.

Smith, L.H. Jr., 1996, Discussion of ASME paper 96GT-029 and ASME paper 96-GT-253, Birmingham, United Kingdom.

Smith, L.H. Jr., 1999, GE Aircraft Engines, Private Communication, CAFMIX II Development.

Turner, M.G., Vitt, P.H., Topp, D.A., Saeidi, S., Hunter, S.D., Dailey, L.D., and Beach, T.A., 1999 , "Multistage Simulations of the GE90 Turbine," To be presented at the ASME IGTI Turbomachinery Conference 1999.

Turner, M.G., 1999, Private Communication, Blockage Estimates.

Valkov, T., and Tan, C.S., 1993, "Control of the Unsteady Flow in a Stator Blade Row Interacting With Upstream Moving Wakes," ASME paper 93-GT-23.

Valkov, T., and Tan, C.S., 1998, "Effect of Upstream Rotor Vortical Disturbances on the Time-Average Performance of Axial Compressor Stators", ASME paper 98-GT312, ASME paper 98-GT-313.

van de Wall, A.G., Kadambi, J.R., Boyle, R.J., and Adamczyk, J.J., 1995, "The Transport of Vorticies Through a Turbine Cascade," ASME paper 95-GT-240.

van de Wall, A.G., 1999, "A Transport Model for the Deterministic Stresses Associated with Turbomachinery Blade Row Interactions," Ph. D Thesis, Department of Mechanical and Aerospace Engineering, Case Western Reserve University.

Van Zante, D.E., Adamczyk, J.J., Strazisar, A.J., and Okiishi, T.H., 1997, "Wake Recovery Performance Benefit in a High-Speed Axial Flow Compressor," ASME paper 97GT-535.

Van Zante, D.E., Strazisar, A.J., Wood, J.R., Hathaway, M.D., and Okiishi, T.H., 1999, "Recommendations for Achieving Accurate Numerical Simulation of Tip Clearance Flow in Transonic Compressor Rotors," To be presented at the ASME IGTI Turbomachinery Conference 1999.

Walker, G.J., 1974, "The Unsteady Nature of Boundary Layer Transition on an Axial Flow Compressor," ASME paper 74-GT-135.

Wellborn, S.R., and Okiishi, T.H., 1996, "Effects of Shrouded Stator Cavity Flows on Multistage Compressor Aerodynamic Performance," NASA CR 198536.

Wellborn, S.R., and Okiishi, T.H., 1998, "The Influence of Shrouded Stator Cavity Flows on Multistage Compressor 
Performance," ASME paper 98-GT-12.

Wellborn, S.R., Tolchinsky, I., and Okiishi, T.H., 1999, "Modeling Shrouded Stator Cavity Flows in Axial-Flow Compressors," To be presented at the ASME IGTI Turbomachinery Conference 1999.

Wellborn, S.R., 1999, Rolls-Royce Allison, Private Communication, Multistage Compressor Simulations.

Wisler, D.C., 1977, "Core Compressor Exit Stage Study:
Vol. 1 - Blading Design," NASA CR-135391.

Wu, C.H., 1952, "A General Theory of Three-Dimensional Flow in Subsonic or Supersonic Turbomachines of Axial,Radial- and Mixed-Flow Type," NACA TN 2604.

Zierke, W.C., Straka, W.A., and Taylor, T.D., 1993, "The High Reynolds Number Flow Through an Axial Flow Pump," Applied Research Lab. Penn. State Univ. T.R. 93-12. 


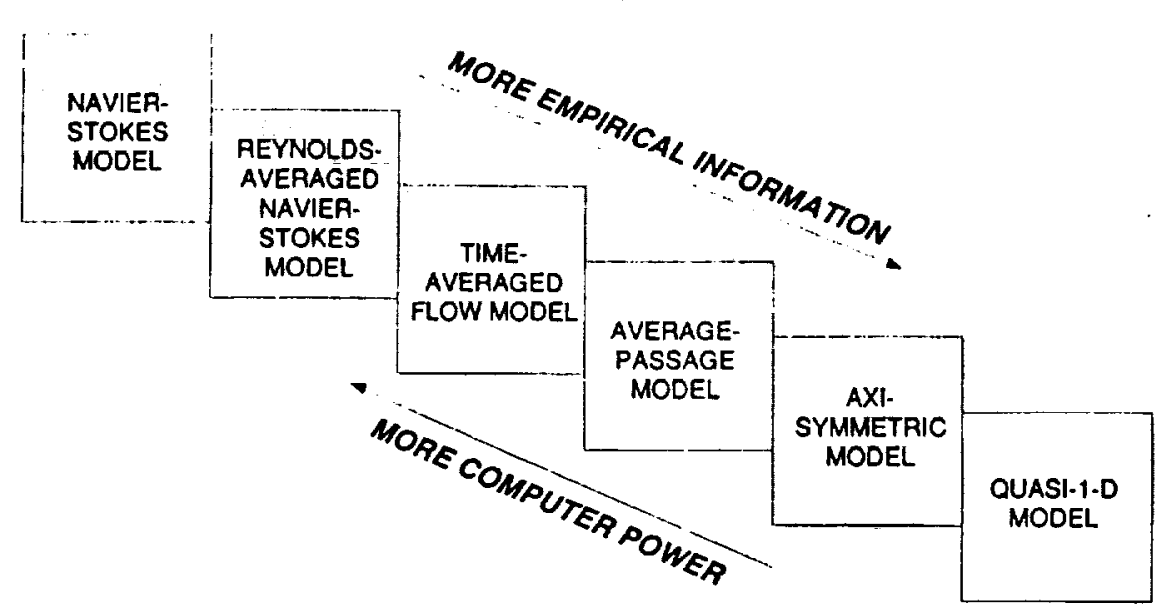

Fig. 1. The range of mathematical models which can be used to simulate turbomachinery flows.

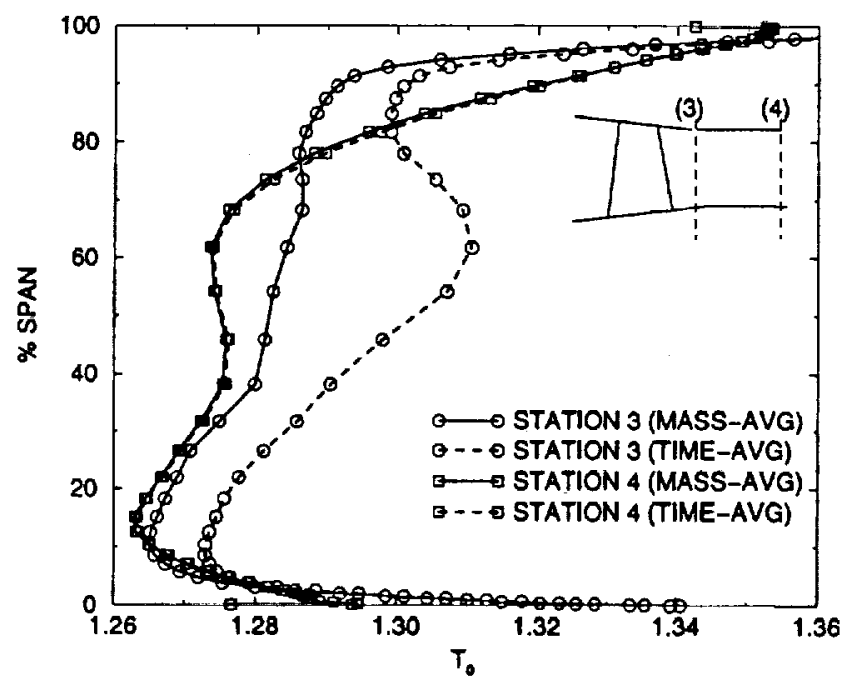

Fig. 2. Spanwise distribution of total temperature downstream of a high speed compressor rotor.

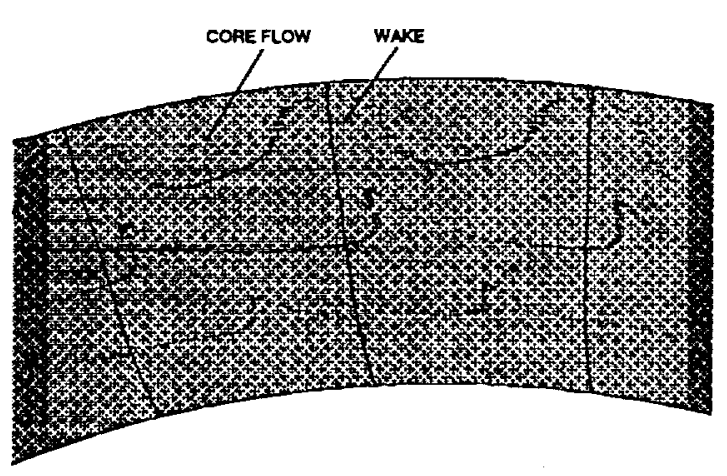

Fig. 3. Radial transport of rotor wake fluid particles. 


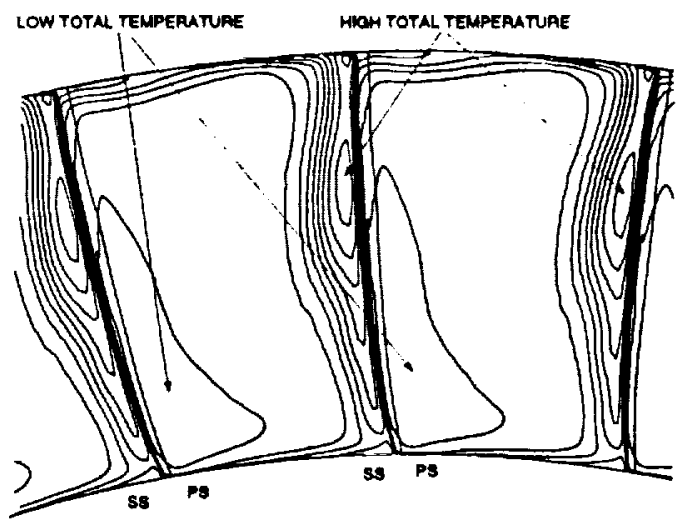

Fig. 4a. Total temperature exiting a high speed compressor rotor at an instant of time.

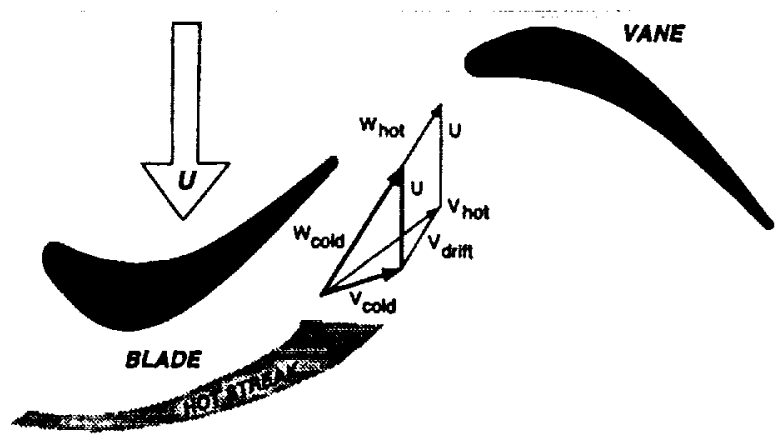

Fig. 5. Kerrebrock and Mikolajczak effect in a turbine stage.
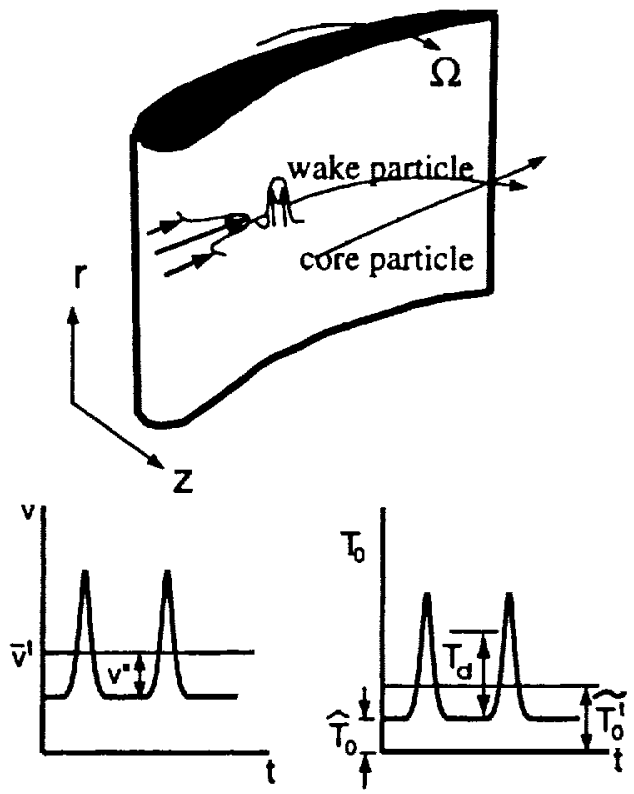

Fig. 4b. Time history of radial velocity and total temperature downstream of a compressor rotor.

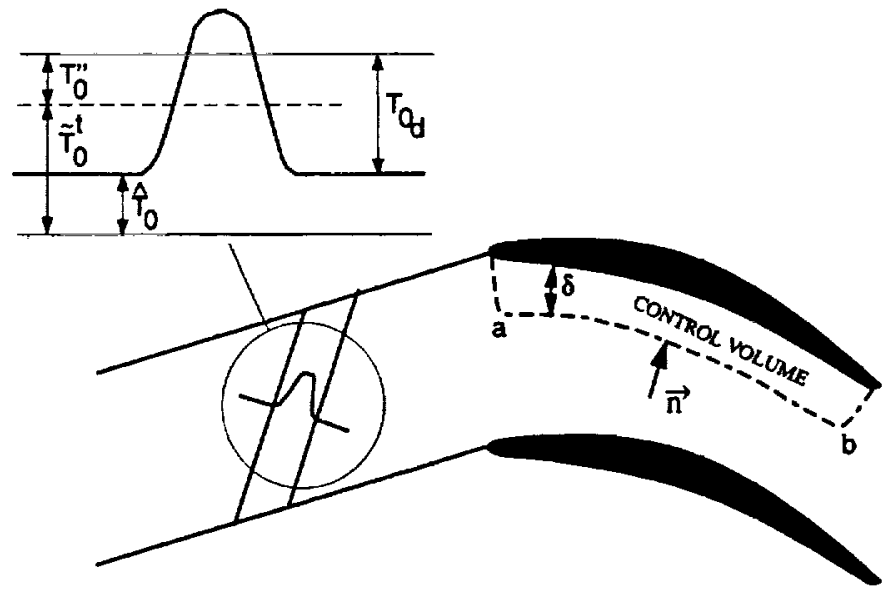

Fig. 6. Convection of a hot streak through a turbine cascade. 

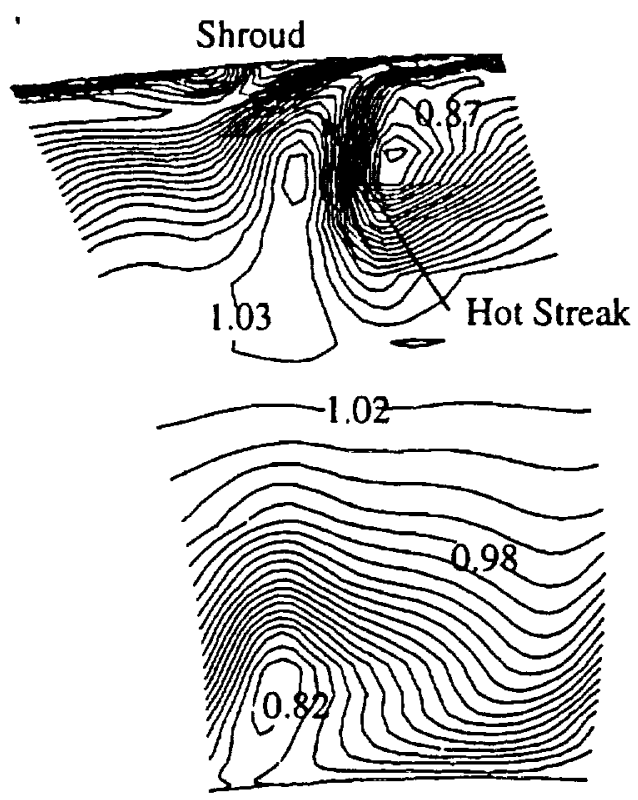

Hub

Fig. 7. Snapshot of unsteady total temperature ratio exiting a turbine rotor.

Fig. 8. Turbine blade temperature distribution.
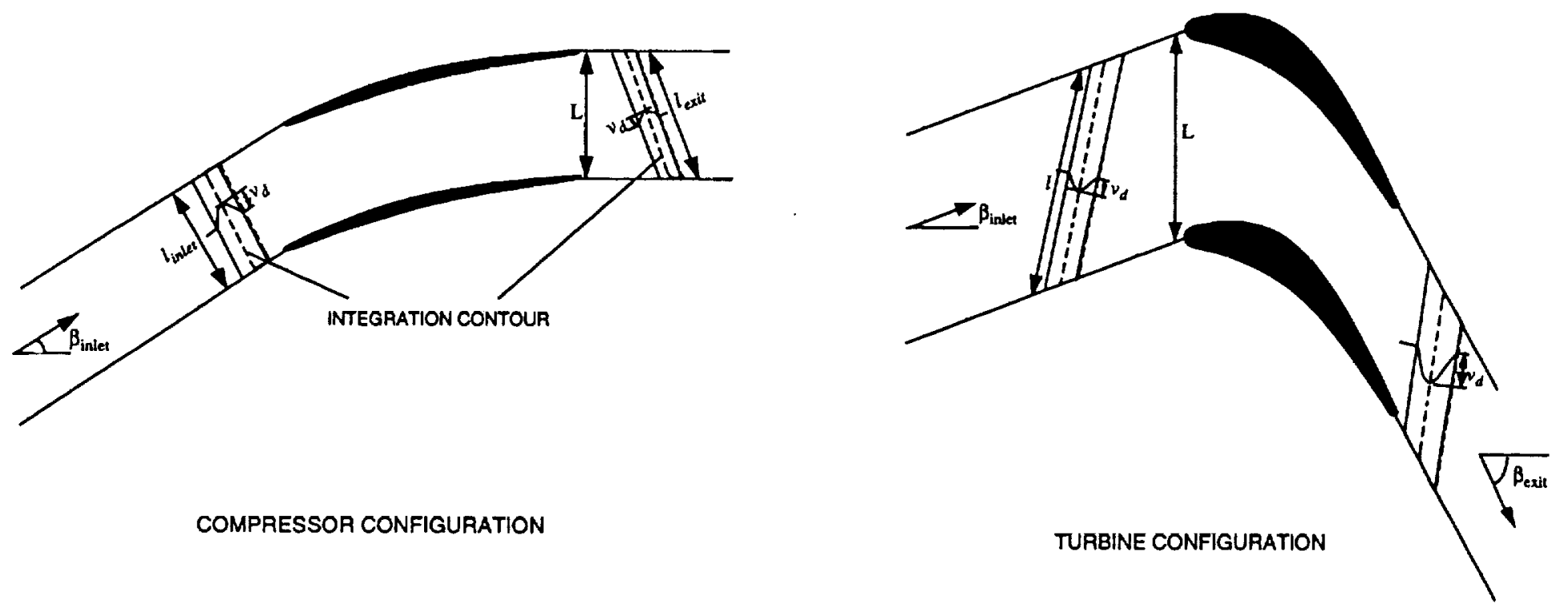

Fig. 9. Wake blade row interactions in a typical turbine and compressor. 


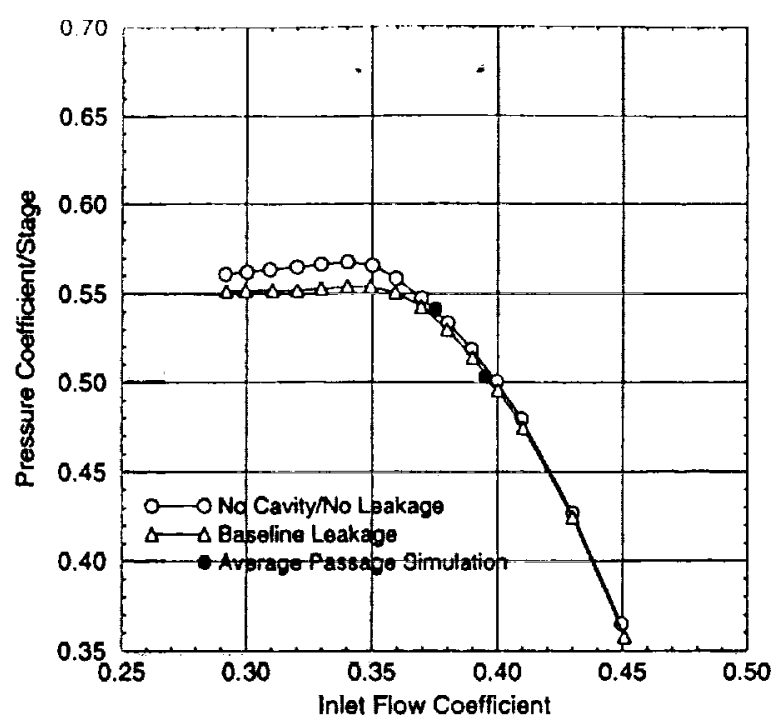

Fig. 10. Overall pressure coefficient for the NASA-Lewis low speed four-stage axial compressor.
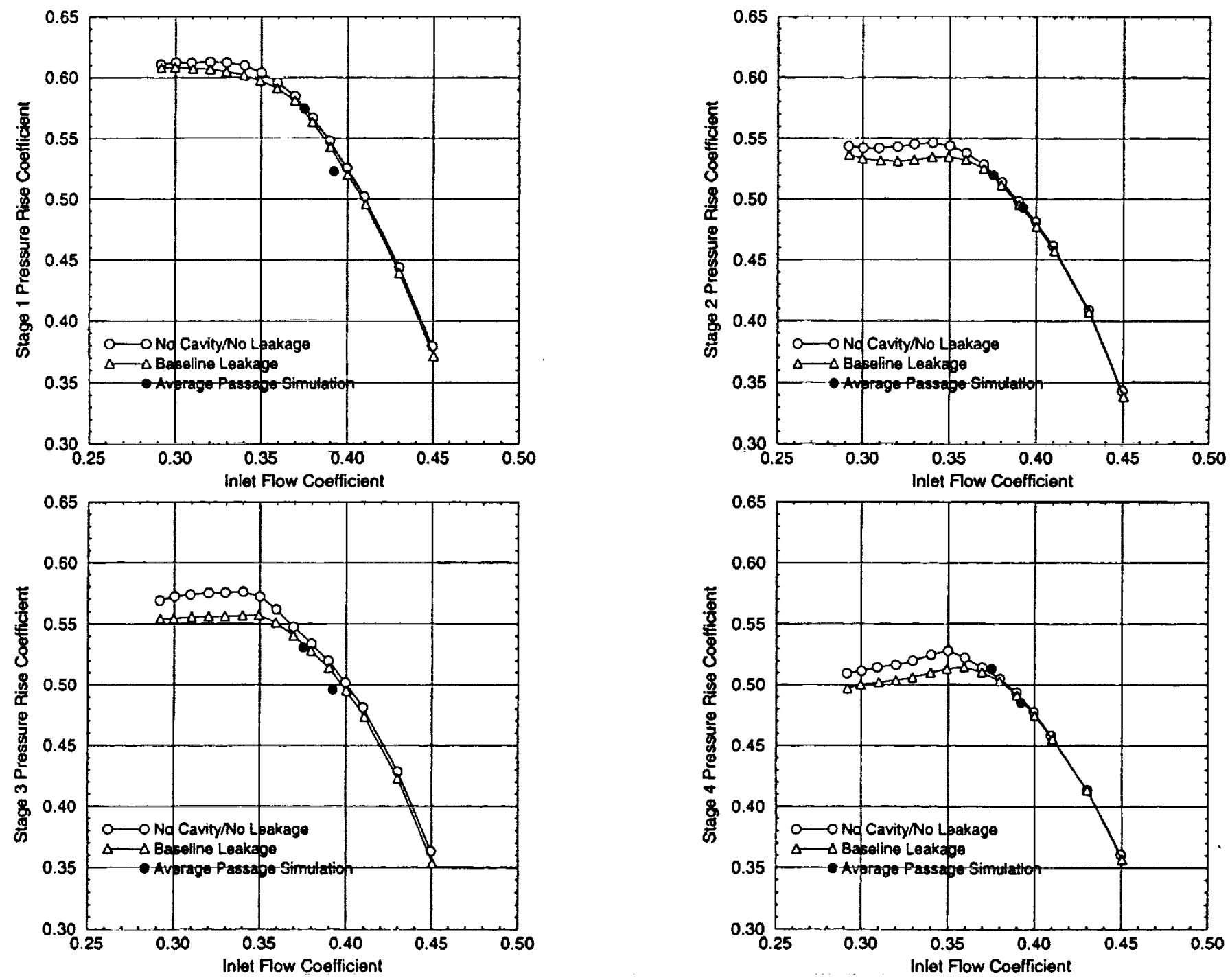

Fig. 11. Individual stage pressure rise coefficient as a function of inlet flow coefficient for the NASA-Lewis low speed four-stage axial compressor. 

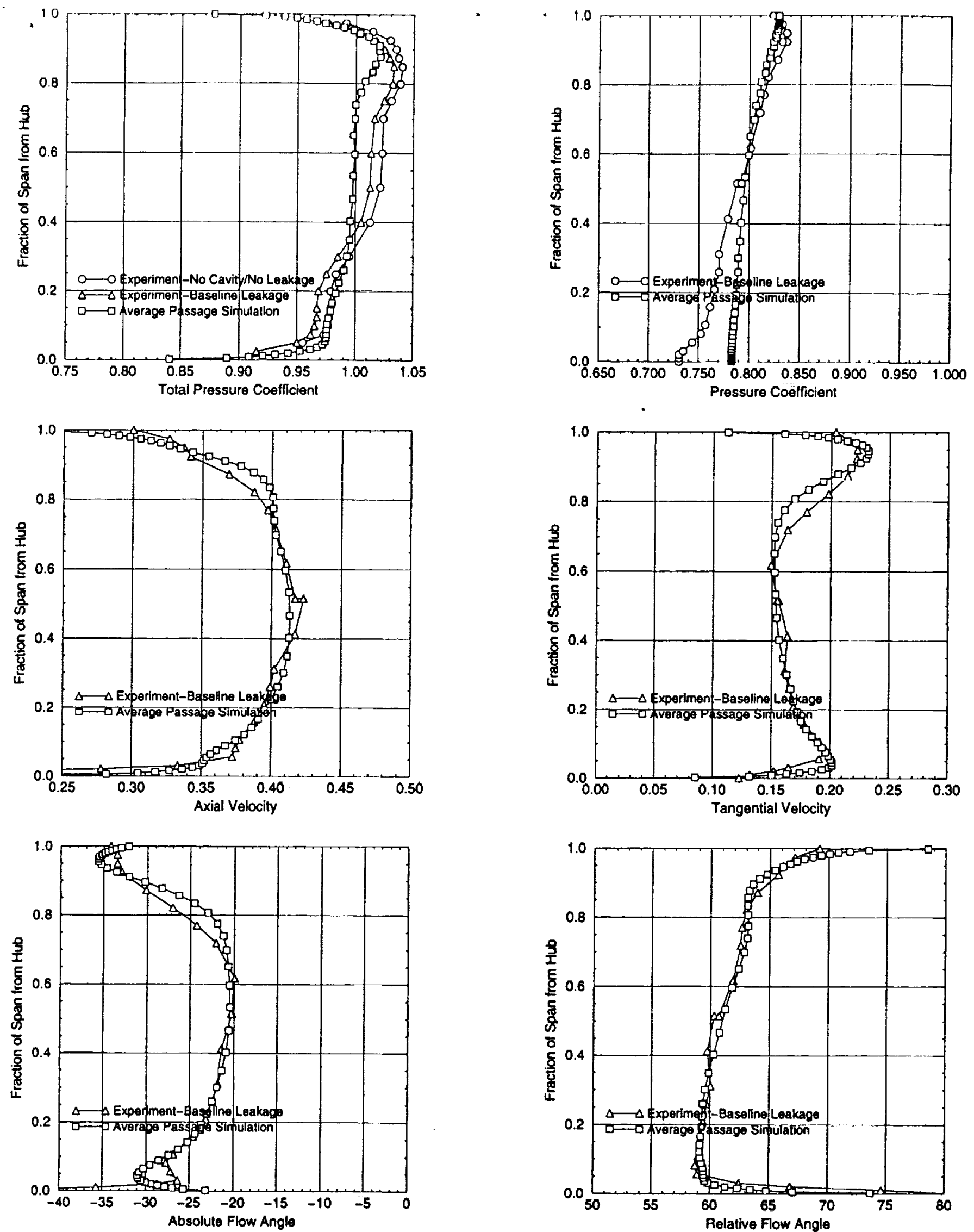

Fig. 12. Axisymmetric flow variables exiting the 2nd stage stator in the NASA-Lewis low speed four-stage axial compressor. 

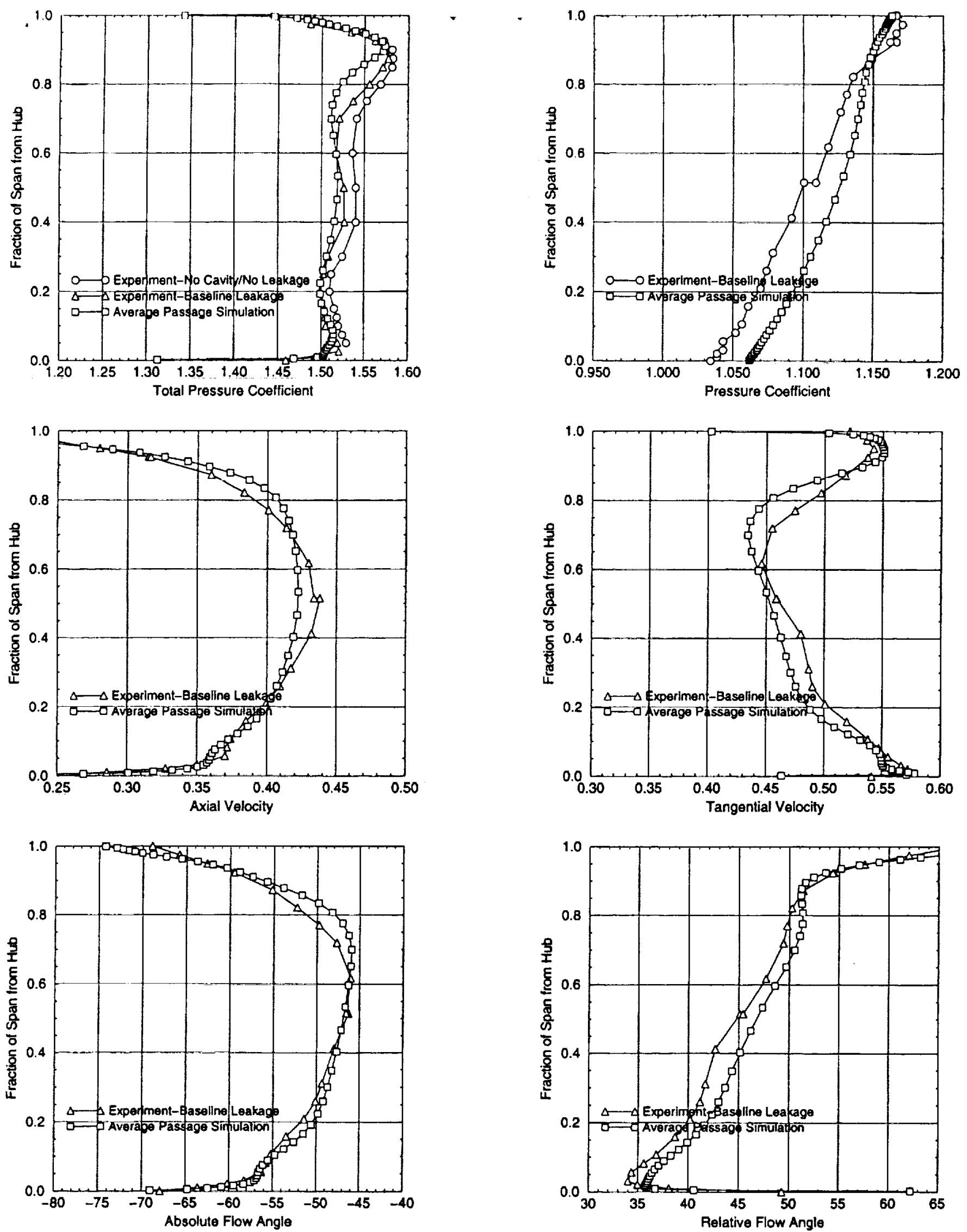

Fig. 13. Axisymmetric flow variables exiting the 3rd stage rotor in the NASA-Lewis low speed four-stage axial compressor. 


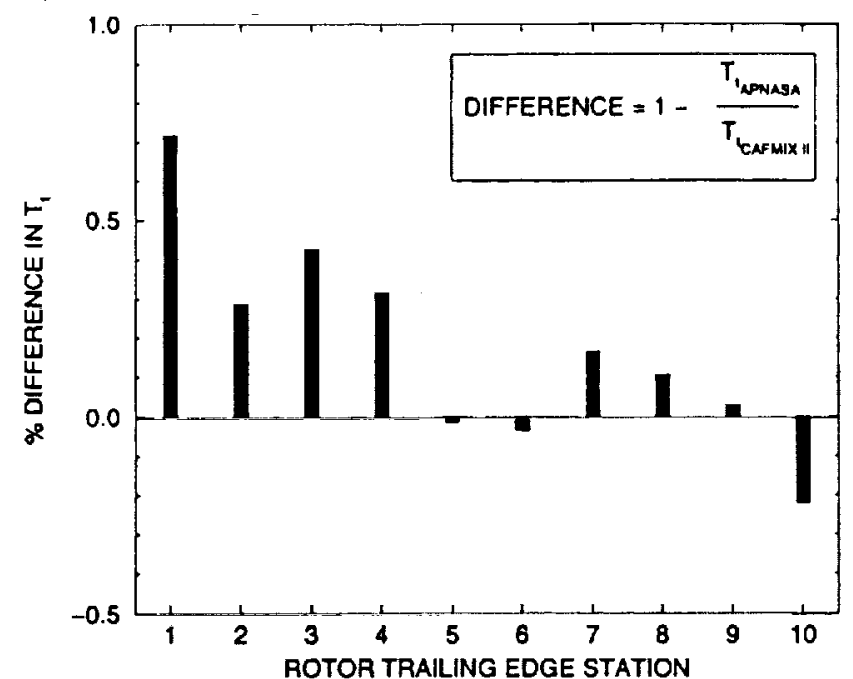

Fig. 14a. Comparison of total temperature as predicted by APNASA and CAFMIX II for the 10-stage GE90 compressor.

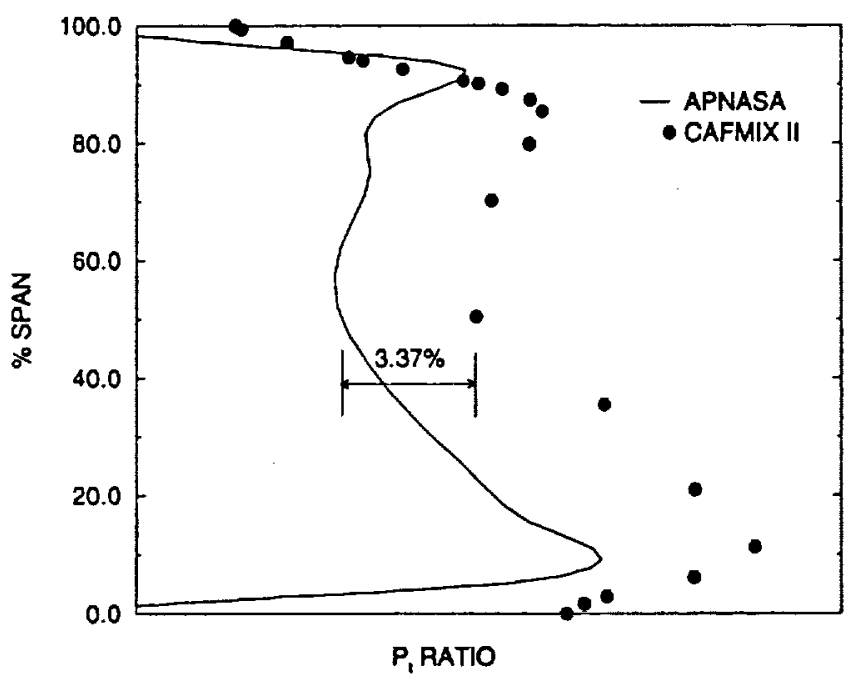

Fig. 15a. Spanwise distribution of total pressure at the exit of the stage 3 stator in the GE90 compressor.

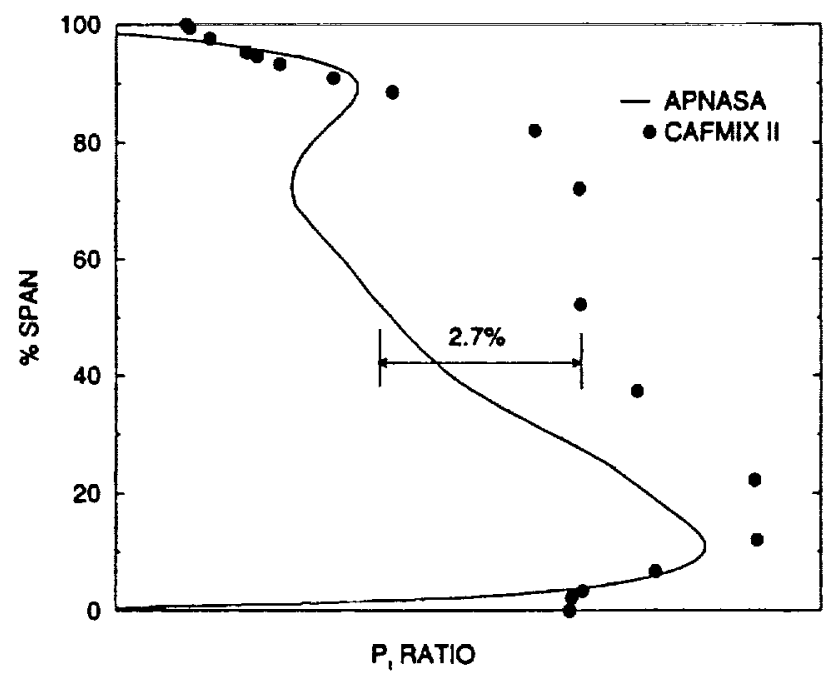

Fig. 15c. Spanwise distribution of total pressure at the exit of the stage 7 stator in the GE90 compressor.

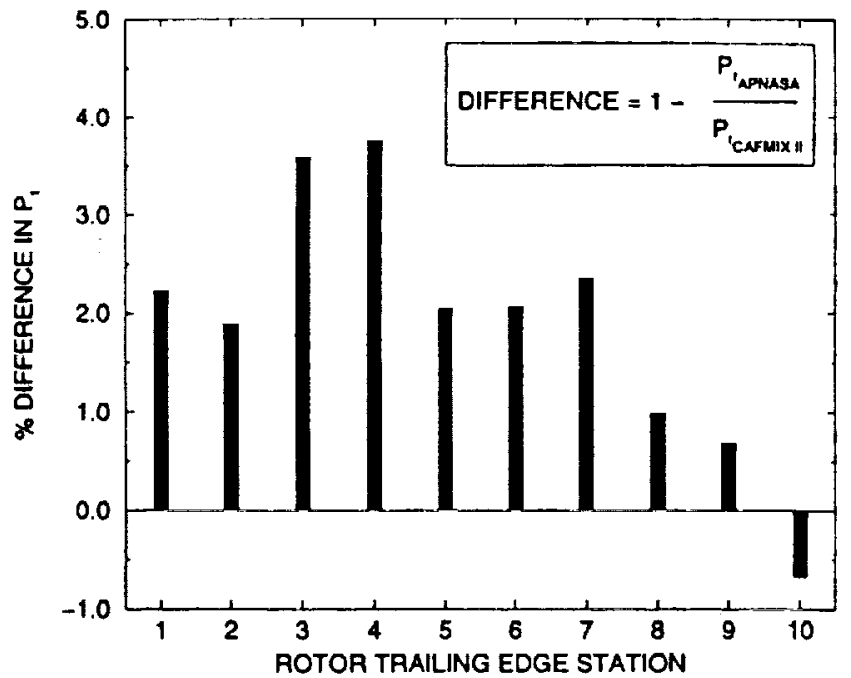

Fig. 14b. Comparison of total pressure as predicted by APNASA and CAFMIX II for the 10-stage GE90 compressor.

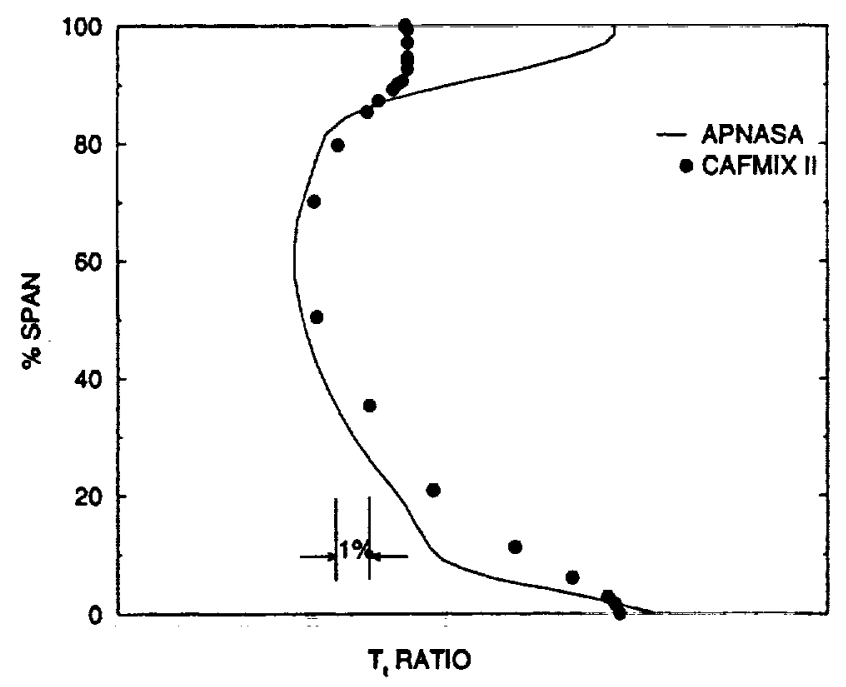

Fig. 15b. Spanwise distribution of total temperature at the exit of the stage 3 stator in the GE90 compressor.

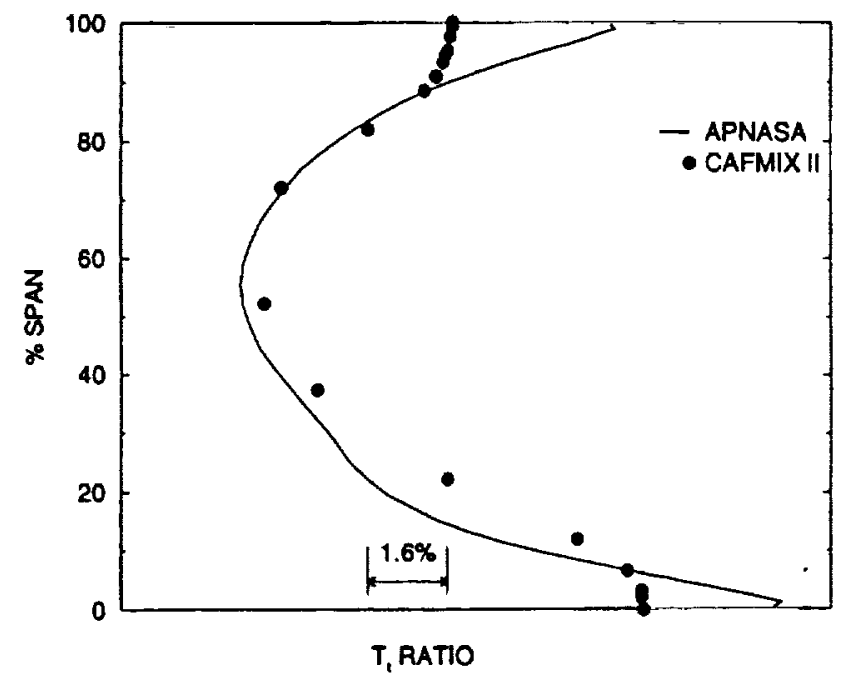

Fig. 15d. Spanwise distribution of total temperature at the exit of the stage 7 stator in the GE90 compressor. 


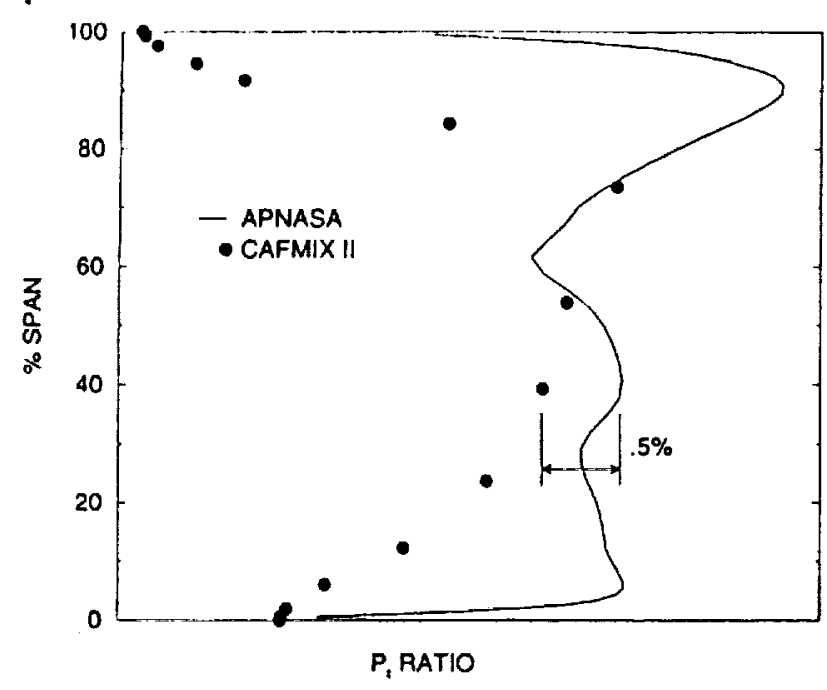

Fig. 15e. Spanwise distribution of total pressure at the compressor exit in the GE90 compressor.

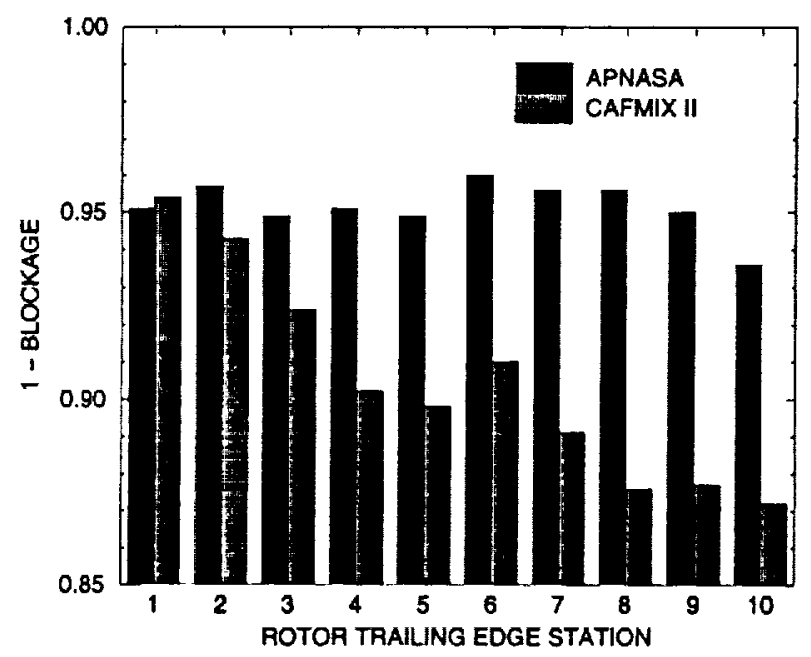

Fig. 16. Flow blockage distribution through the GE90 compressor.

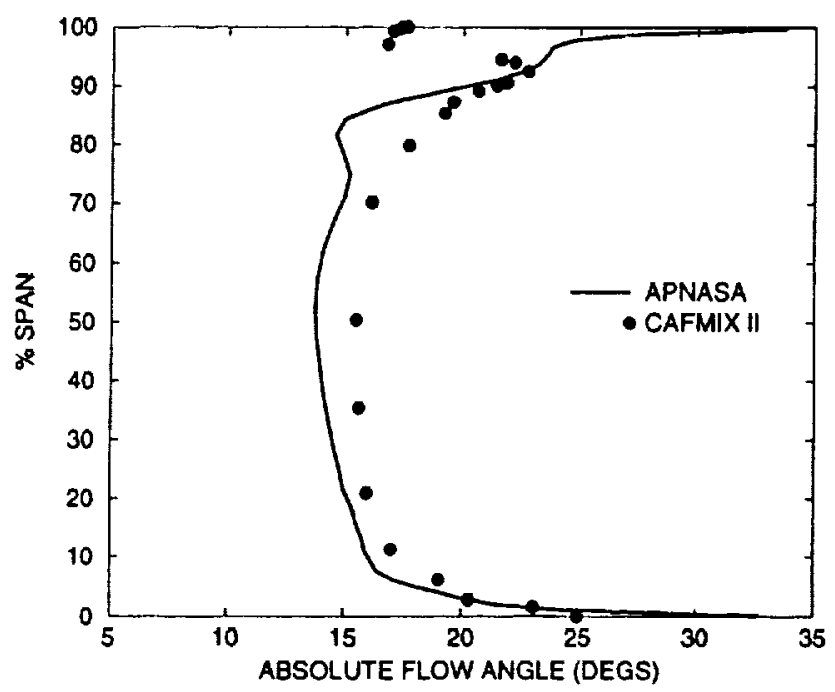

Fig. 17b. Absolute fow angle at the exit of the stage 3 stator in the GE90 compressor.

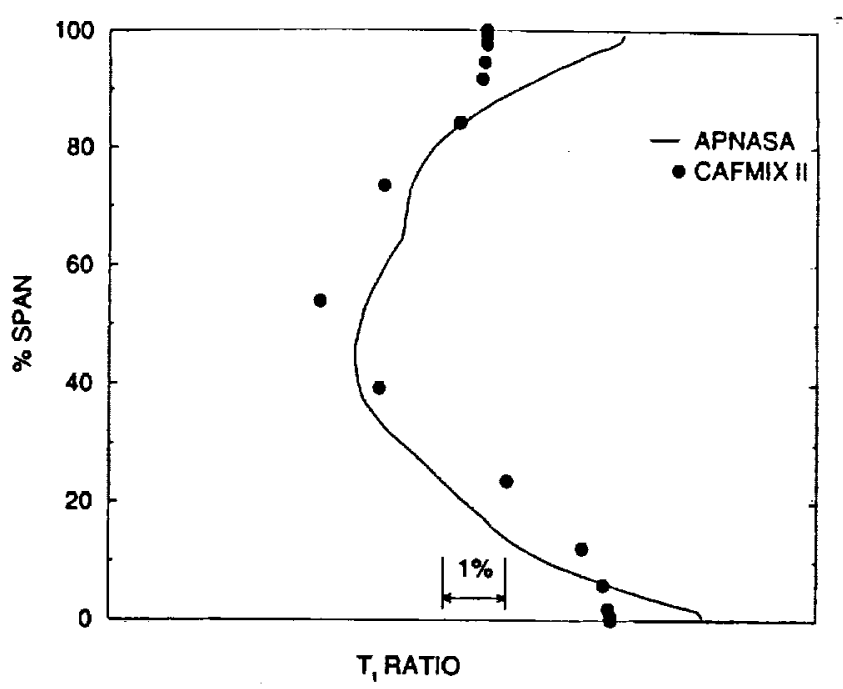

Fig. 15e. Spanwise distribution of total pressure at the compressor exit in the GE90 compressor.

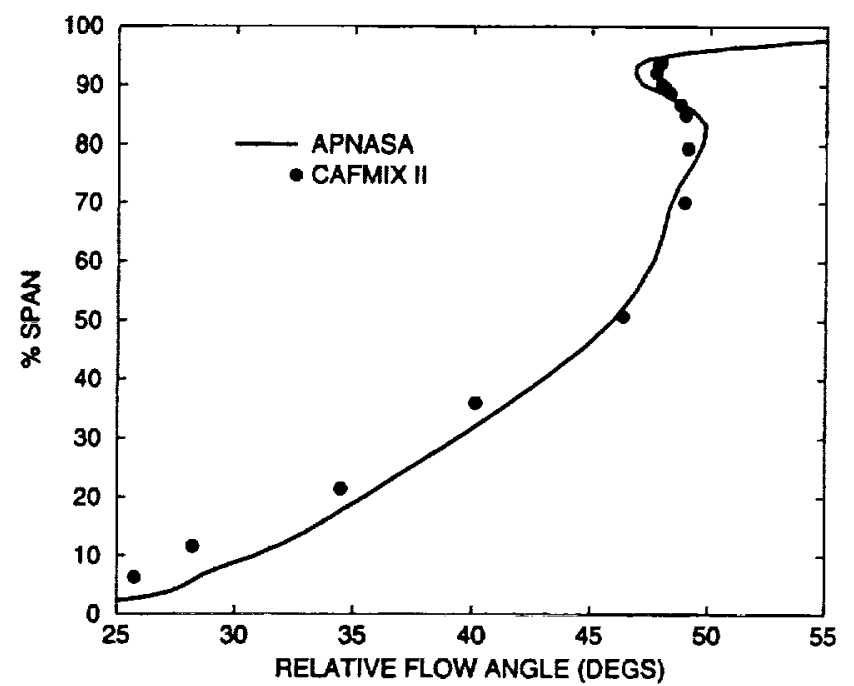

Fig. 17a. Relative flow angle at the exit of the stage 3 rotor in the GE90 compressor.

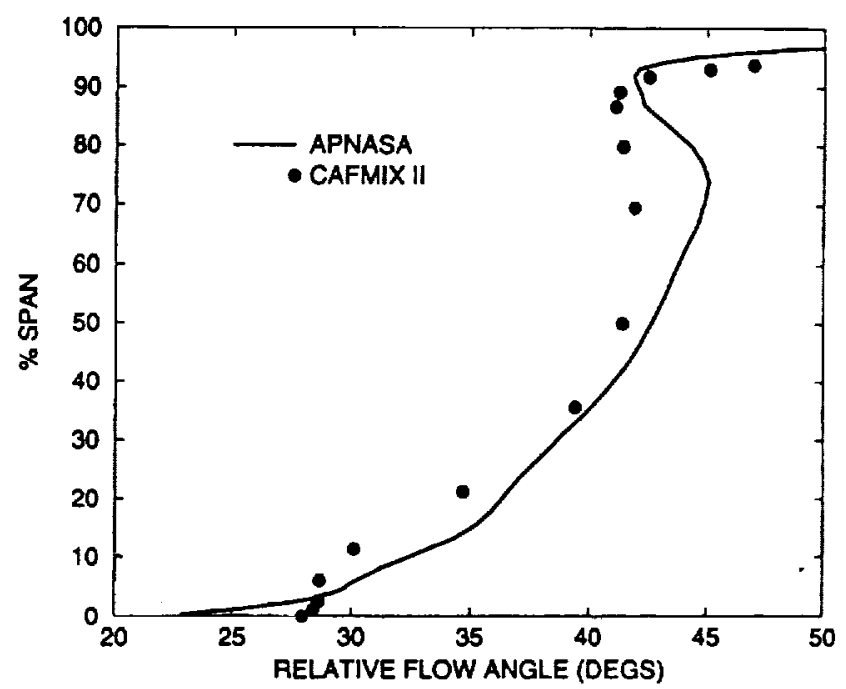

Fig. 17c. Relative flow angle at the exit of the stage 7 rotor in the GE90 compressor. 


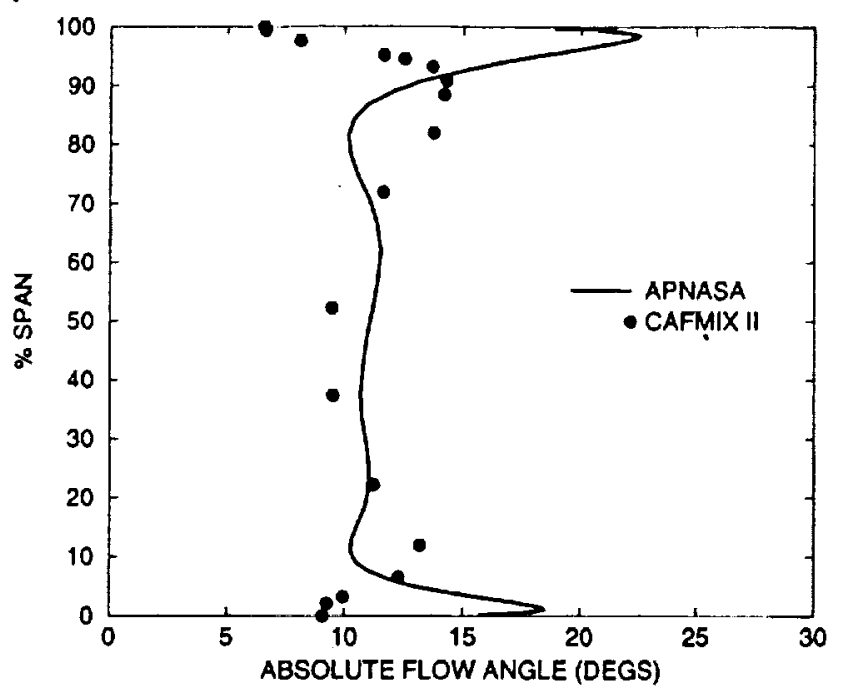

Fig. 17d. Absolute flow angle at the exit of the stage 7 stator in the GE90 compressor.

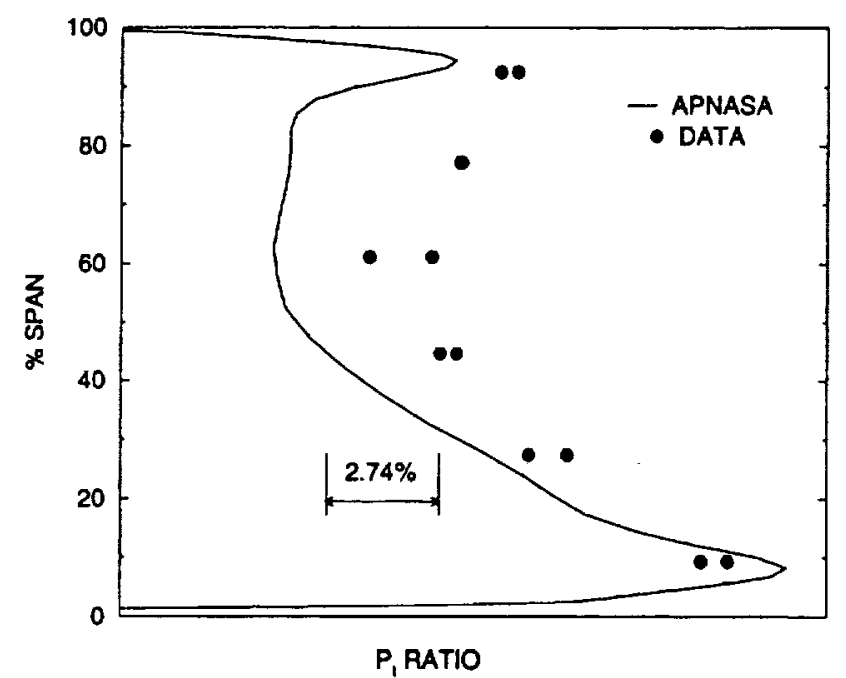

Fig. 19a. Spanwise distribution of total pressure at the exit of the stage 3 rotor in the GE90 near design speed.

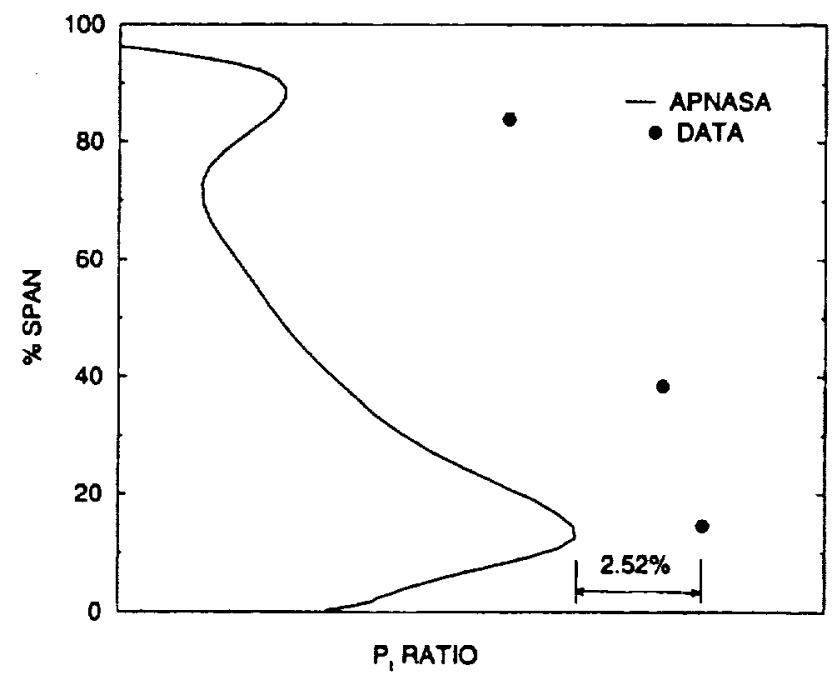

Fiy. 19c. Spanwise distribution of total pressure at the exit of the stage 7 rotor in the GE90 near design speed.

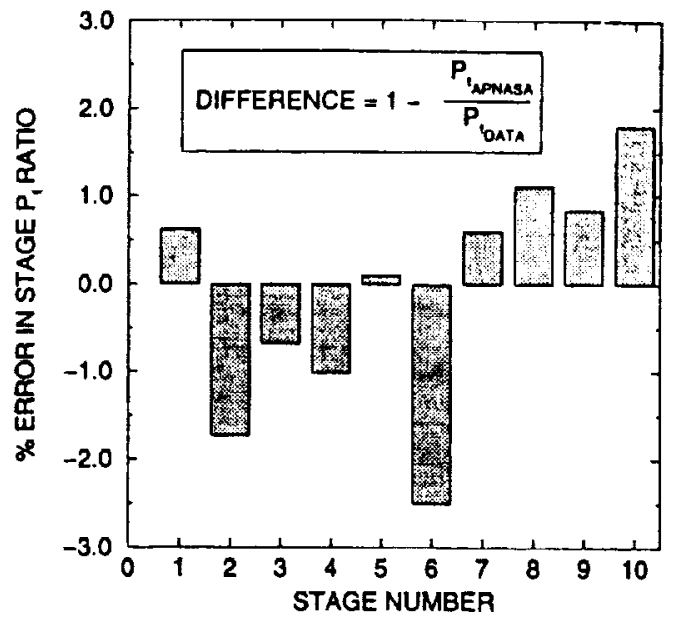

Fig. 18. Percent difference in total pressure through the GE90 compressor near the design speed operating point.

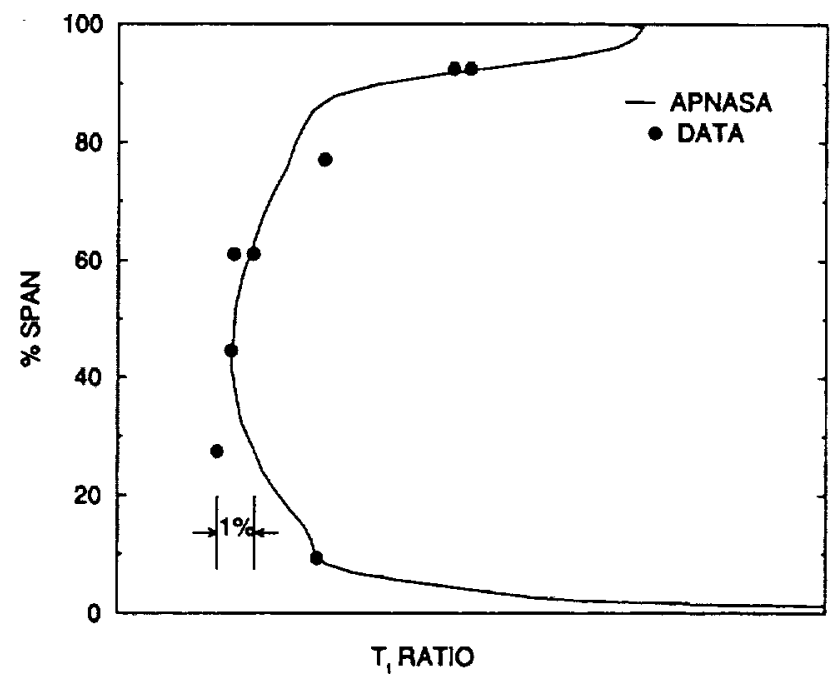

Fig. 19b. Spanwise distribution of total temperature at the exit of the stage 3 rotor in the GE90 near design speed.

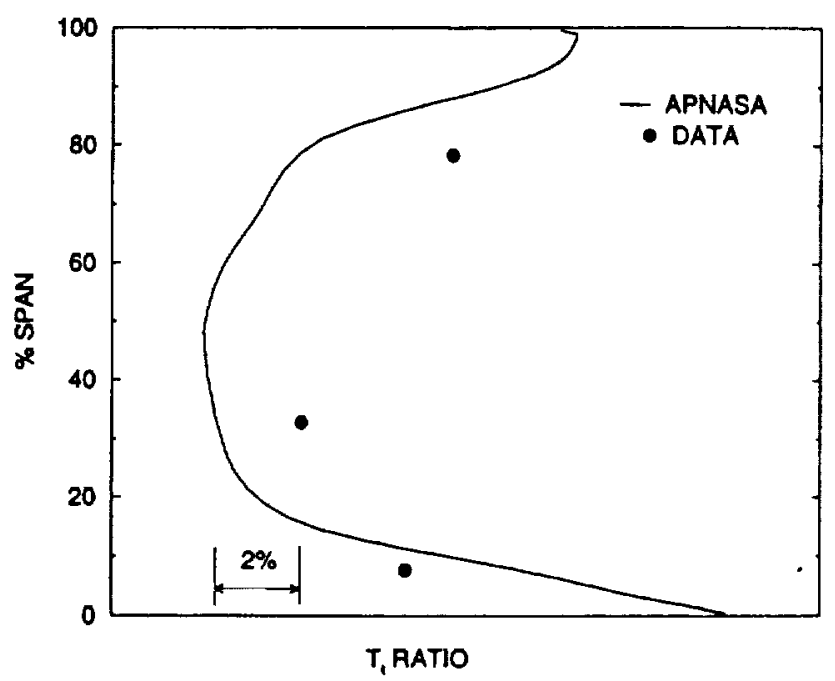

Fig. 19d. Spanwise distribution of total pressure at the exit of the stage 7 rotor in the GE90 near design speed. 


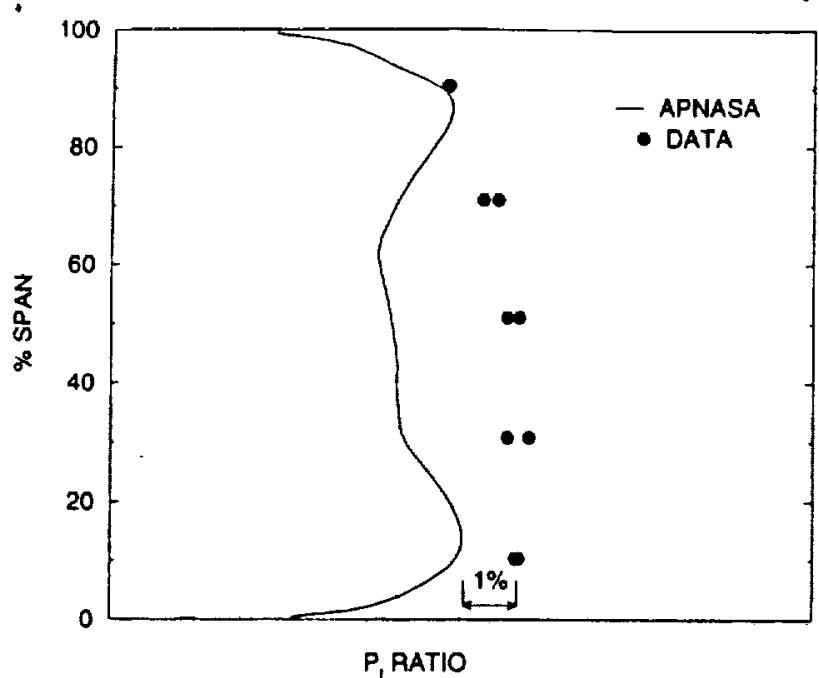

Fig. 19e. Spanwise distribution of total pressure at the exit of the GE90 near design speed.

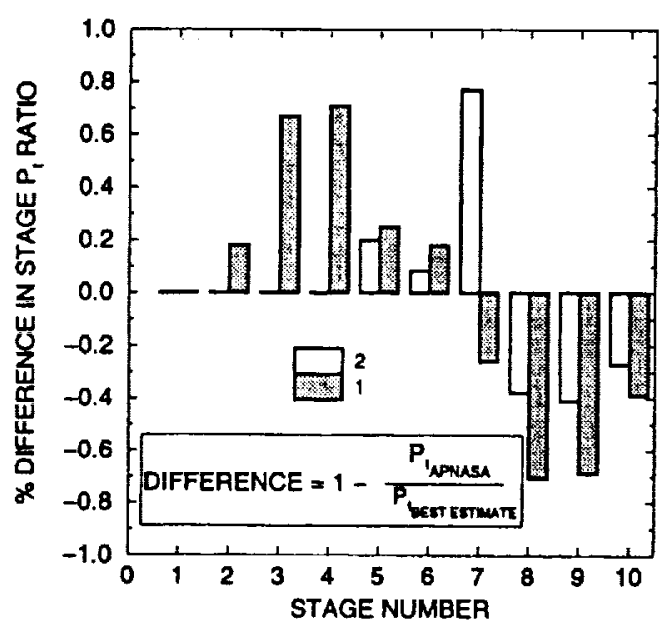

Fig. 20. Percent difference in stage pressure ratio as a function of bleed rates for the GE90 compressor.

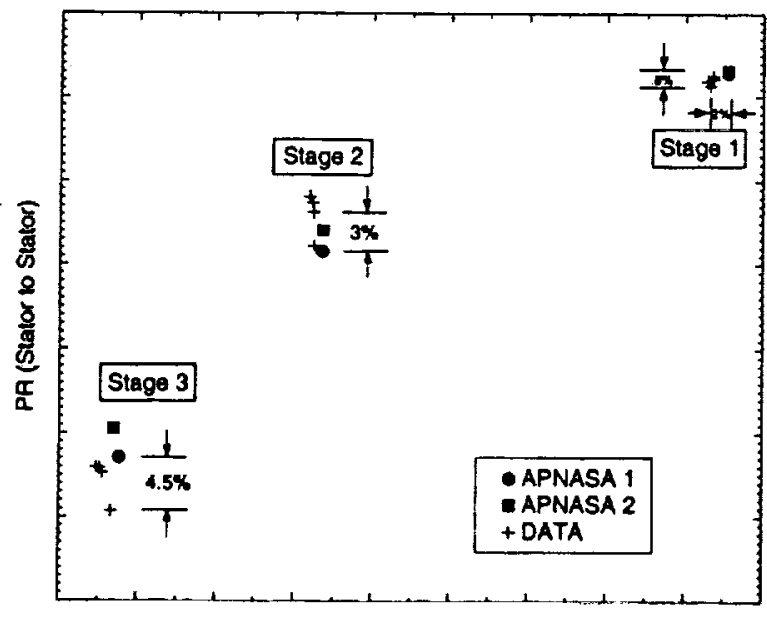

Stator Intel Wcorr

Fig. 22a. Stage total pressure ratio characteristic as a function of corrected mass flow for a 3-stage high speed compressor.

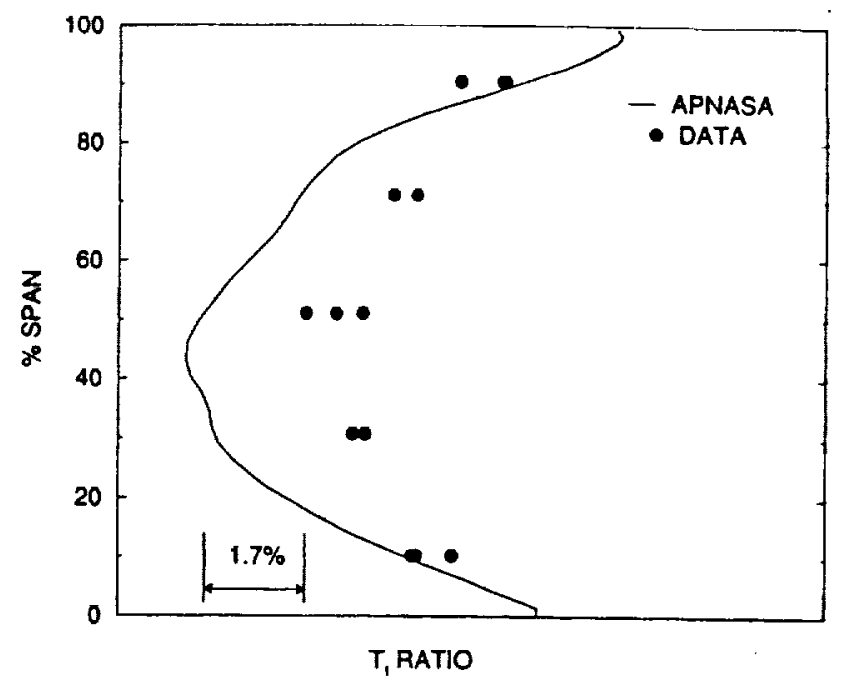

Fig. 19f. Spanwise distribution of total temperature at the exit of the GE90 near design speed.

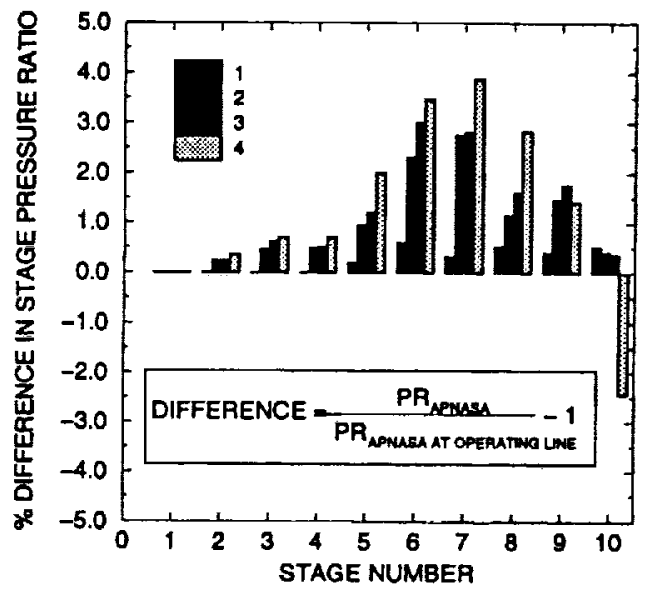

Fig. 21. Percent difference in stage pressure ratio as a function of back pressure for the GE90 compressor.

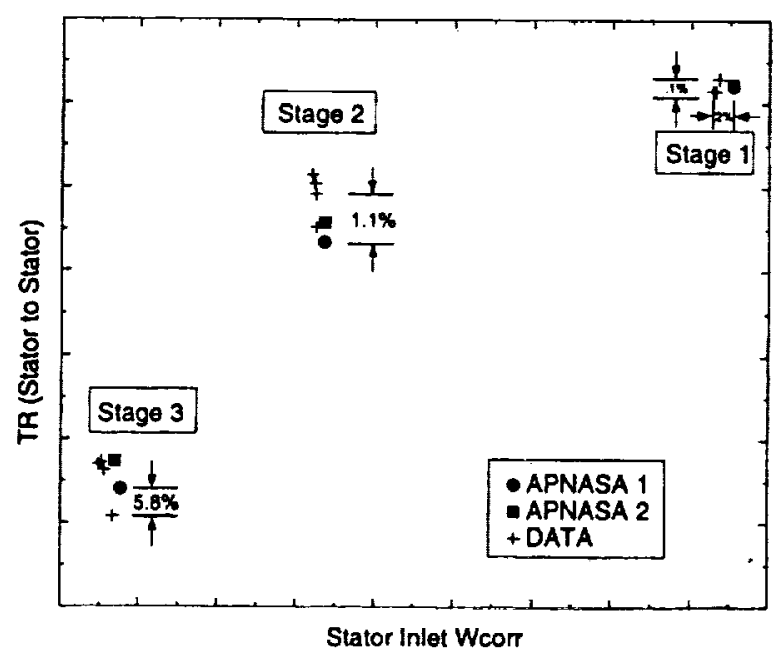

Fig. 22b. Stage total temperature ratio characteristic as a function of corrected mass flow for a 3-stage high speed compressor. 


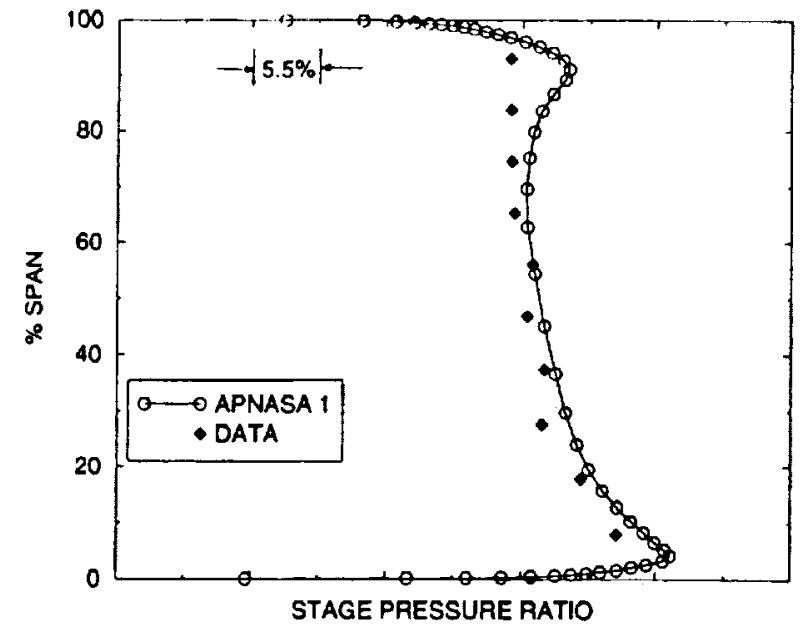

Fig. 23a. Spanwise distribution of total pressure at the exit of the stage 1 rotor for a 3-stage high speed compressor.

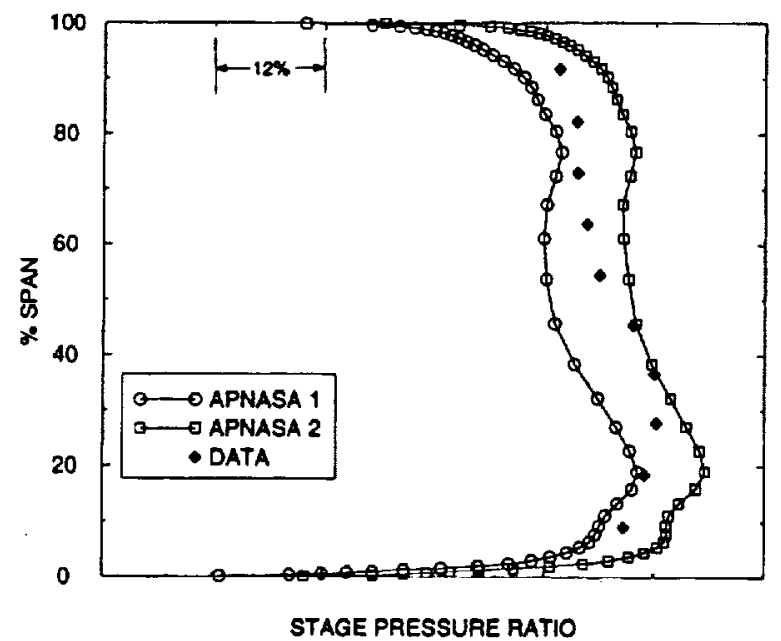

Fig. 24a. Spanwise distribution of total pressure at the exit of the stage 3 rotor for a 3 -stage high speed compressor.

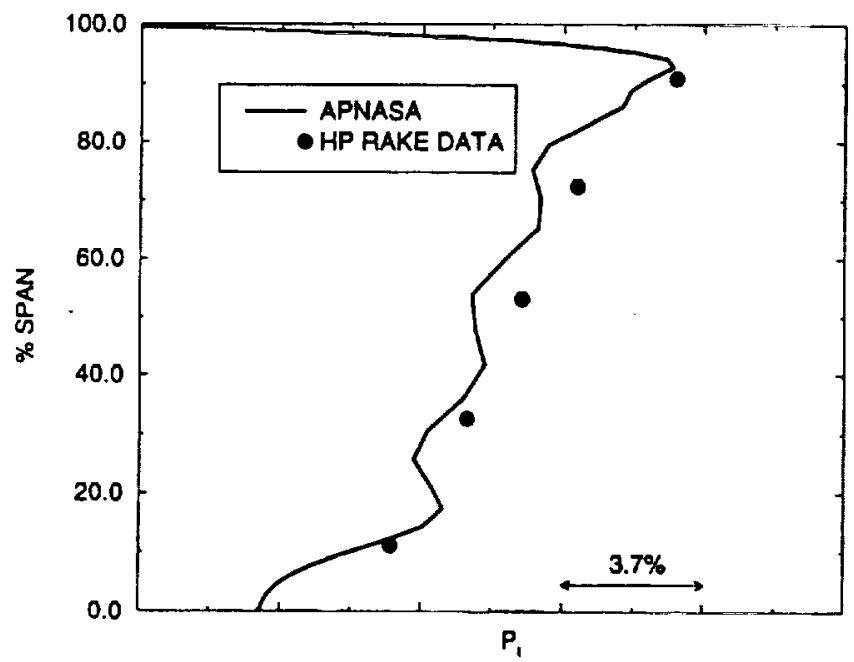

Fig. 25a. Total pressure profile at the exit of an HP turbine stage 2 rotor.

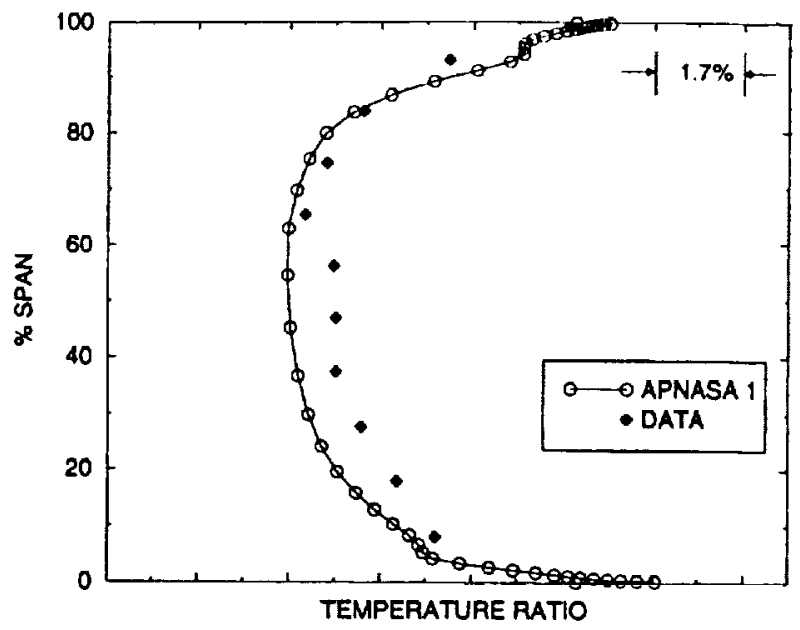

Fig. 23b. Spanwise distribution of total temperature at the exit of the stage I rotor for a 3-stage high speed compressor.

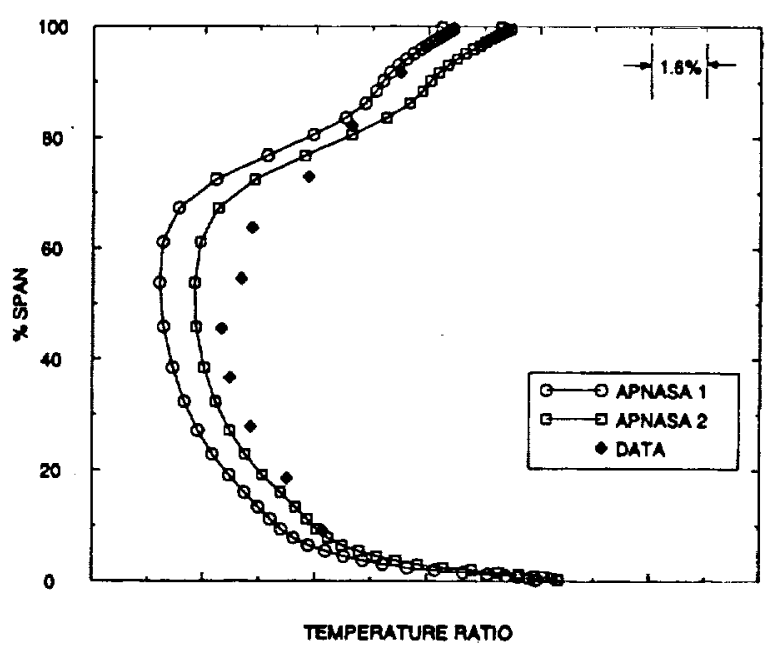

Fig. 24b. Spanwise distribution of total temperature at the exit of the stage 3 rotor for a 3-stage high speed compressor.

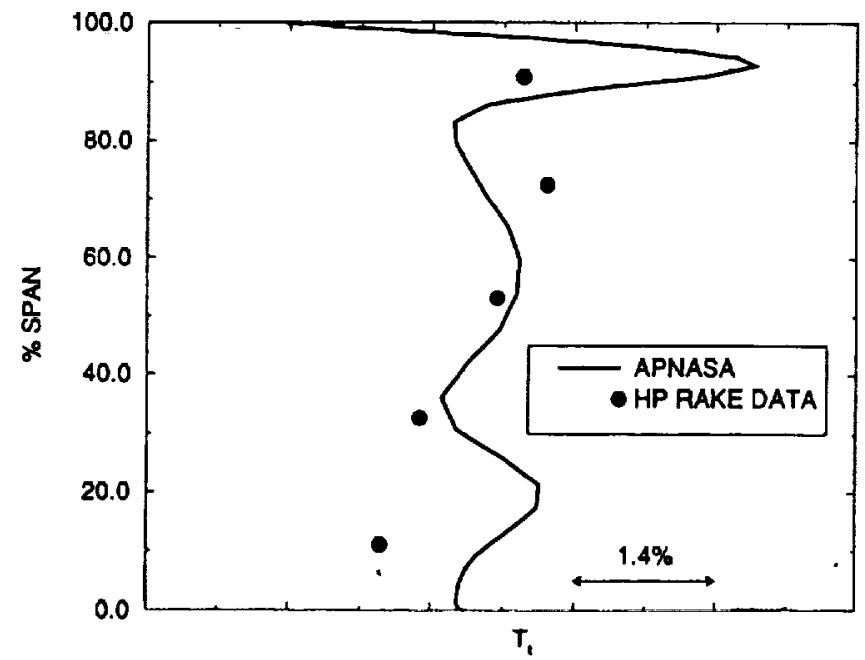

Fig. 25b. Total temperature profile at the exit of an HP turbine stage 2 rotor. 


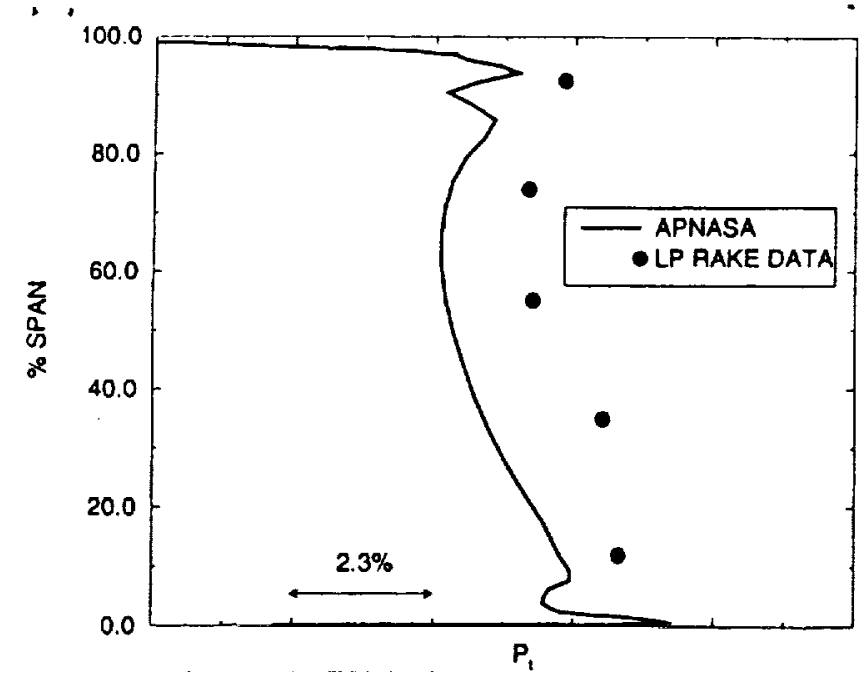

Fig. 26a. Total pressure profile at the exit of an LP turbine.

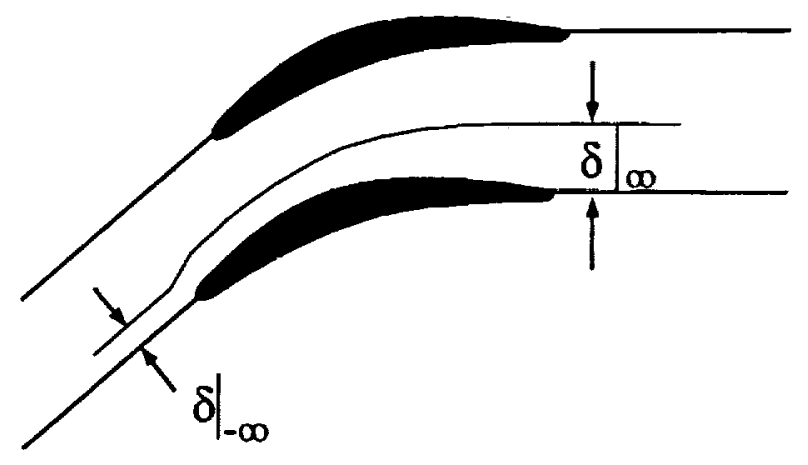

Fig. 27. Steady flow through a cascade with blockage.

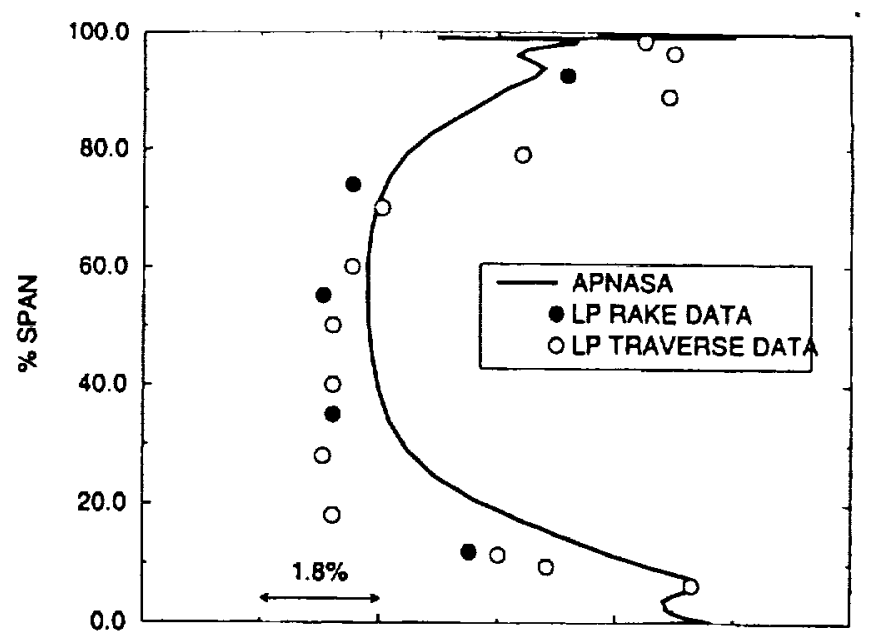

Fig. 26b. Total temperature profile at the exit of an LP turbine.

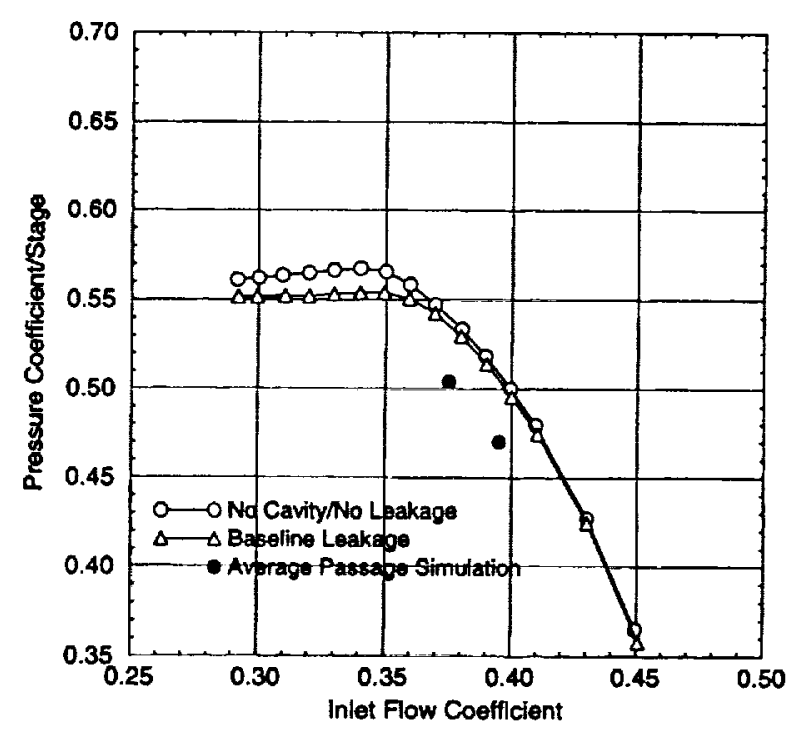

Fig. 28. Overall pressure coefficient as a function of inlet flow coefficient for the NASA-Lewis four stage axial flow compressor. 